\title{
Vadose Zone Remediation Assessment: M-Area Process Sewer Soil Vapor Extraction Units 782-5M, 782-7M and 782-8M
}

\author{
February 2001 \\ B. D. Riha \\ D. G. Jackson \\ W. K. Hyde \\ B. B. Looney \\ J. Rossabi
}


This document was prepared in conjunction with work accomplished under Contract No.

DE-AC09-96SR18500 with the U.S. Department of Energy.

\section{DISCLAIMER}

This report was prepared as an account of work sponsored by an agency of the United States Government. Neither the United States Government nor any agency thereof, nor any of their employees, makes any warranty, express or implied, or assumes any legal liability or responsibility for the accuracy, completeness, or usefulness of any information, apparatus, product or process disclosed, or represents that its use would not infringe privately owned rights. Reference herein to any specific commercial product, process or service by trade name, trademark, manufacturer, or otherwise does not necessarily constitute or imply its endorsement, recommendation, or favoring by the United States Government or any agency

thereof. The views and opinions of authors expressed herein do not necessarily state or reflect those of the United States Government or any agency thereof.

This report has been reproduced directly from the best available copy.

Available for sale to the public, in paper, from: U.S. Department of Commerce, National Technical Information Service, 5285 Port Royal Road, Springfield, VA 22161, phone: (800)

553-6847, fax: (703) 605-6900, email: orders@ntis.fedworld.gov online ordering: http://www.ntis.gov/ordering.htm

Available electronically at http://www.doe.gov/bridge

Available for a processing fee to U.S. Department of Energy and its contractors, in paper, from: U.S. Department of Energy, Office of Scientific and Technical Information, P.O. Box 62, Oak Ridge, TN 37831-0062, phone: (865 ) 576-8401, fax: (865) 576-5728, email: reports@ adonis.osti.gov 


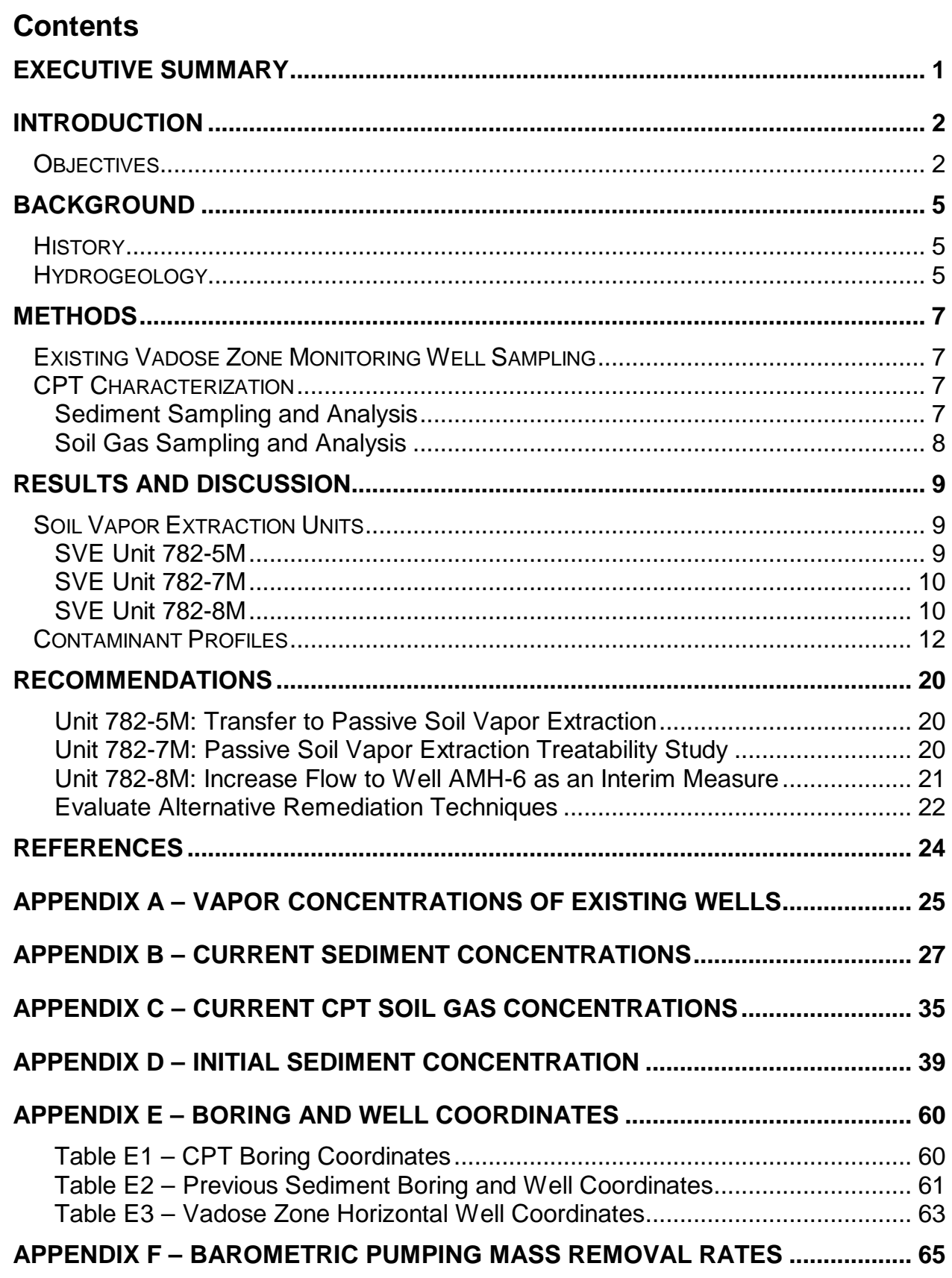




\section{List of Tables}

Table 1 - SVE Unit 782-8M Operation History........................................................ 11

Table 2 - Tabulated Data Descriptions .............................................................. 12

Table 3 - Existing Four-Inch Diameter Wells Near 782-7M .................................... 21

\section{List of Figures}

Figure 1 - Boring and Well Locations ................................................................. 3

Figure 2 - Three Dimensional Image of Boring and Sediment Sample Locations ..... 4

Figure 3 - Schematic of the A/M Area Vadose Zone............................................ 6

Figure 4 - Concentration and Removal Rates for SVE Unit 782-5M ........................ 9

Figure 5 - Concentration and Removal Rates for SVE Unit 782-7M..................... 10

Figure 6 - Concentration and Removal Rates for SVE Unit 782-8M...................... 11

Figure 7 - Initial Maximum Total Sediment Concentration ..................................... 14

Figure 8 - Current Maximum Total Sediment Concentration................................... 15

Figure 9 - PCE Soil Gas Concentration.............................................................. 16

Figure 10 - TCE Soil Gas Concentration ........................................................ 17

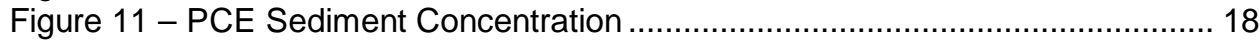

Figure 12 - TCE Sediment Concentration ........................................................ 19

Figure 13 - Proposed Locations for New Vadose Zone Wells ................................. 23 


\section{Executive Summary}

This study focuses on the status of the vadose zone remediation along $1600 \mathrm{ft}$ of the process sewer line between the M-Area security fence and the M-Area settling basin. Three soil vapor extraction (SVE) units $782-5 \mathrm{M}, 782-7 \mathrm{M}$, and $782-8 \mathrm{M}$, connected to 4 vertical wells and 3 horizontal wells have been addressing the vadose zone volatile organic contamination (VOC) since 1995. The specific objectives of this study were to obtain soil gas and sediment samples, evaluate SVE units and vadose zone remediation, and make recommendations to address further remediation needs.

The three SVE units have removed approximately $91,500 \mathrm{lbs}$ of contamination from the vadose zone along the process sewer line. The SVE has effectively cleaned up the central sandy unit (320 to $265 \mathrm{ft} \mathrm{msl}$ ) with contaminant removal from the lower permeability units dictated by mass transfer limitations. Units $782-5 \mathrm{M}$ and $782-8 \mathrm{M}$ have been shut down due to low contaminant removal rates. Unit $782-7 \mathrm{M}$ is currently operating with a removal rate of about $25 \mathrm{lbs} /$ week.

Concentration measurements were made on 60 existing vadose zone monitoring points in the area. Twenty cone penetrometer characterization borings were completed in November of 2000 for soil gas and sediment sampling. A total of 282 sediment samples and 155 soil gas samples were collected.

The following results are drawn from evaluating the concentrations and three dimensional digital images of the sediment and soil gas data:

- A significant amount of contamination has been removed from the vadose zone.

- DNAPL does not exist in the zones sampled $(<200,000 \mu \mathrm{g} / \mathrm{kg}$ TCE and $<50,000$ $\mu \mathrm{g} / \mathrm{kg}$ PCE)

- Residual contamination remains in the fine grain zones, which are not directly addressed by SVE (above and below the central sandy unit).

- Low permeability zones, perched water and cemented zones in the lower fine grain zone (270 foot clay) reduce the effectiveness of SVE from approximately $100 \mathrm{ft}$ down to the water table.

- The remediation between wells MVE-7 and AMH-2 is not being addressed adequately by the current SVE systems.

- The horizontal wells associated with SVE Unit 782-8M have not fully addressed the vadose zone contamination.

In general, the following recommendations address residual contamination that remains in the fine grain sediments where remediation is regulated by mass transfer rates:

- Transfer wells associated with SVE Unit $782-5 \mathrm{M}$ to passive soil vapor extraction (PSVE).

- Perform a PSVE treatability study using wells associated with SVE Unit 782-7M.

- Install three vent wells to increase subsurface flow along the sewer line towards the basin to enhance remediation and provide a 'pressure barrier' between the basin and the PSVE systems.

- Continue to evaluate alternative remediation techniques. 


\section{Introduction}

This study focuses on the status of the vadose zone remediation along $1600 \mathrm{ft}$ of the process sewer line between the M-Area security fence and the M-Area settling basin. Three soil vapor extraction (SVE) units $782-5 \mathrm{M}, 782-7 \mathrm{M}$, and $782-8 \mathrm{M}$, connected to 4 vertical wells and 3 horizontal wells have been addressing the vadose zone volatile organic contamination (VOC) in these areas since 1995. This report will address removal rates of the current soil vapor extraction systems, compare initial and current contamination profiles, and propose methods to control and remediate the remaining contamination. A plan view of the study area is shown in Figure 1. This map shows the location of the old process sewer, initial and current sampling locations, monitoring wells and vapor extraction wells with corresponding SVE units.

\section{Objectives}

The primary objective of this assessment was to evaluate the vadose zone remediation along the M-Area process sewer line according to the $\mathrm{A} / \mathrm{M}$ Area Vadose Zone Monitoring Plan (Jarosch et al., 1998). The specific objectives were to:

- Obtain soil gas and sediment samples to evaluate vadose zone remediation

- Borings were located along the process sewer where worst case contamination was expected.

- Borings were located at apparent data gaps.

- Borings were located near pre-remediation borings for comparison.

- The fine grained layers were targeted for sediment samples since these zones are not directly addressed by SVE and serve to trap residual VOCs.

- At each location soil gas samples were taken at approximately $10 \mathrm{ft}$ intervals.

- Target sampling depth was $120 \mathrm{ft}$.

- Evaluate remediation effectiveness by comparing historical sediment and soil gas concentration data to data collected during the last quarter of 2000.

- Evaluate contaminant removal by the SVE units.

- Make recommendations to address further remediation needs.

A three-dimensional image showing the locations of the cone penetrometer borings and sediment samples in relation to the process sewer and remediation wells is provided in Figure 2 for reference. 


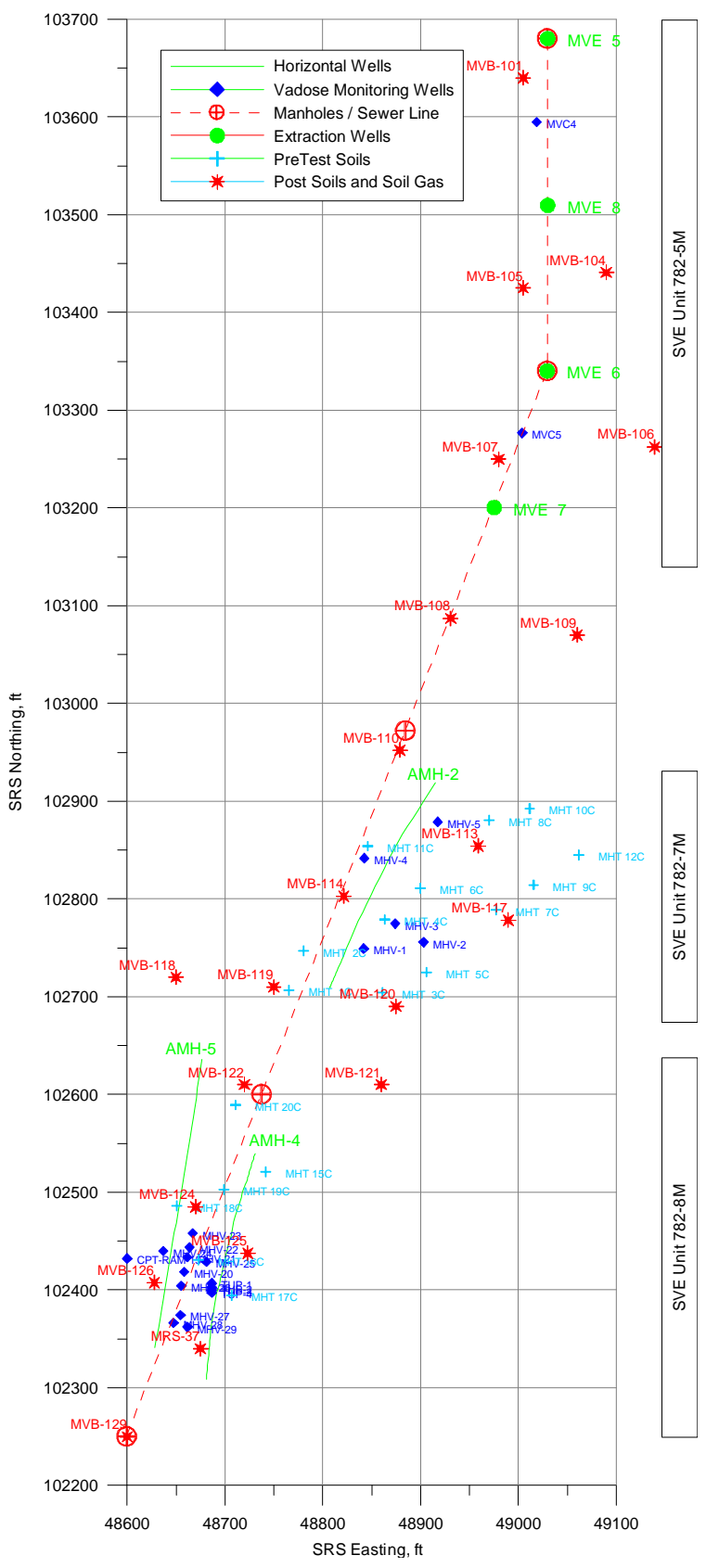

Figure 1 - Boring and Well Locations 


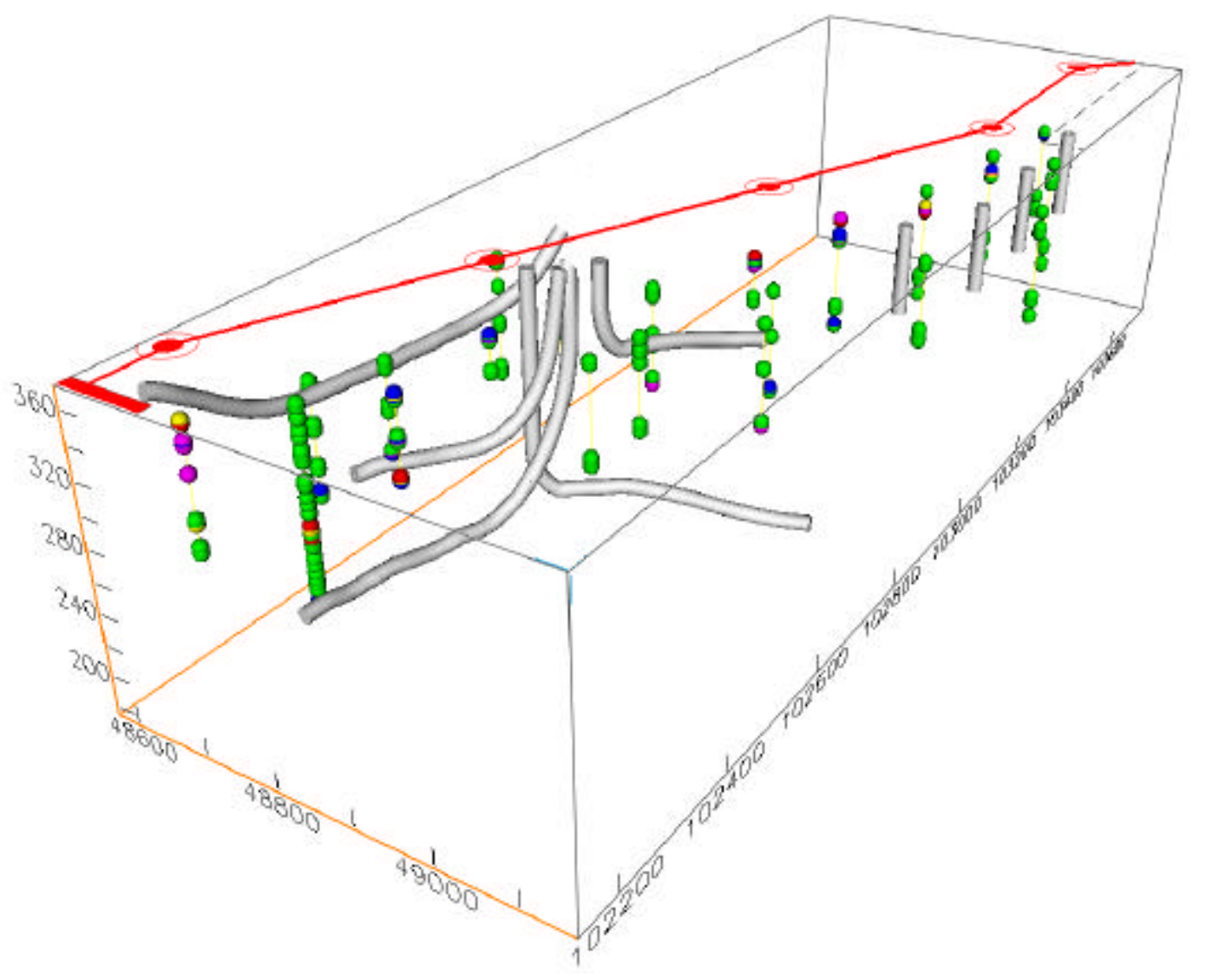

Figure 2 - Three Dimensional Image of Boring and Sediment Sample Locations 


\section{Background}

\section{History}

The Savannah River Site (SRS) is a Department of Energy (DOE) facility located in South Carolina on the Savannah River. Previous operations and waste disposal in the Administration and Metallurgical fabrication areas (A/M Areas) at SRS resulted in the release of chlorinated solvents to the subsurface. The contaminants are primarily trichloroethylene (TCE) and tetrachloroethylene (PCE). Previous investigations document and provide additional Information on the release, characterization, and remediation history of the M-Area vadose zone.(Jarosch et al., 1997; Jarosch et al., 1998)

\section{Hydrogeology}

The sediments within the A/M Area vadose zone consist of sand, sandy clay, clayey sand, and clay deposited from the middle to upper Eocene in shallow marine, lagoonal, or fluvial environments. These lithologies while typically deposited as layers or wedges are often discontinuous due to depositional or post depositional processes (i.e. erosion).

Eddy and others (Eddy et al., 1991) identified four semi-confining/confining zones in this area. The upper three zones are semi-confining, clay rich or interbedded zones all above or within the M-Area aquifer zone. These are from the top down the "325 foot clay", "300 foot clay", and "270 ft clay" zones. The 325 foot clay and 300 foot clay are in the vadose zone and impact the flow of gasses above the water table and recharge of air and water from the ground surface. The 270 foot clay is an interbedded zone that extends below the water table. The fourth, lowermost zone is named the "200 foot clay" confining zone and corresponds to the "Green Clay" confining zone of the Steed Pond aquifer. The Green Clay separates the M-Area or water table aquifer from the Lost Lake aquifer zone. A schematic diagram of the A/M Area vadose zone is provided in Figure 3(Jarosch et al., 1997).

Recent lithologic and facies mapping studies have examined the lateral continuity and vertical competency of these units. (Parker et al., 1999) In this investigation, structure contours and isopach maps of the vadose zone lithologic units were developed. Within each unit the distribution of mud fraction was examined using qualitative and quantitative techniques. The distribution of the arithmetic average mud fraction within each unit was used to provide information on the lateral continuity. The vertical competency was evaluated based upon the distribution of the geometric mean and the standard deviation in mud fraction in the vertical direction. With the exception of the "230-foot clay", Smits and others (1999) follow the previous lithologic nomenclature (Eddy et al., 1991) for the vadose zone. 
WSRC-TR-2001-00077

February 2001

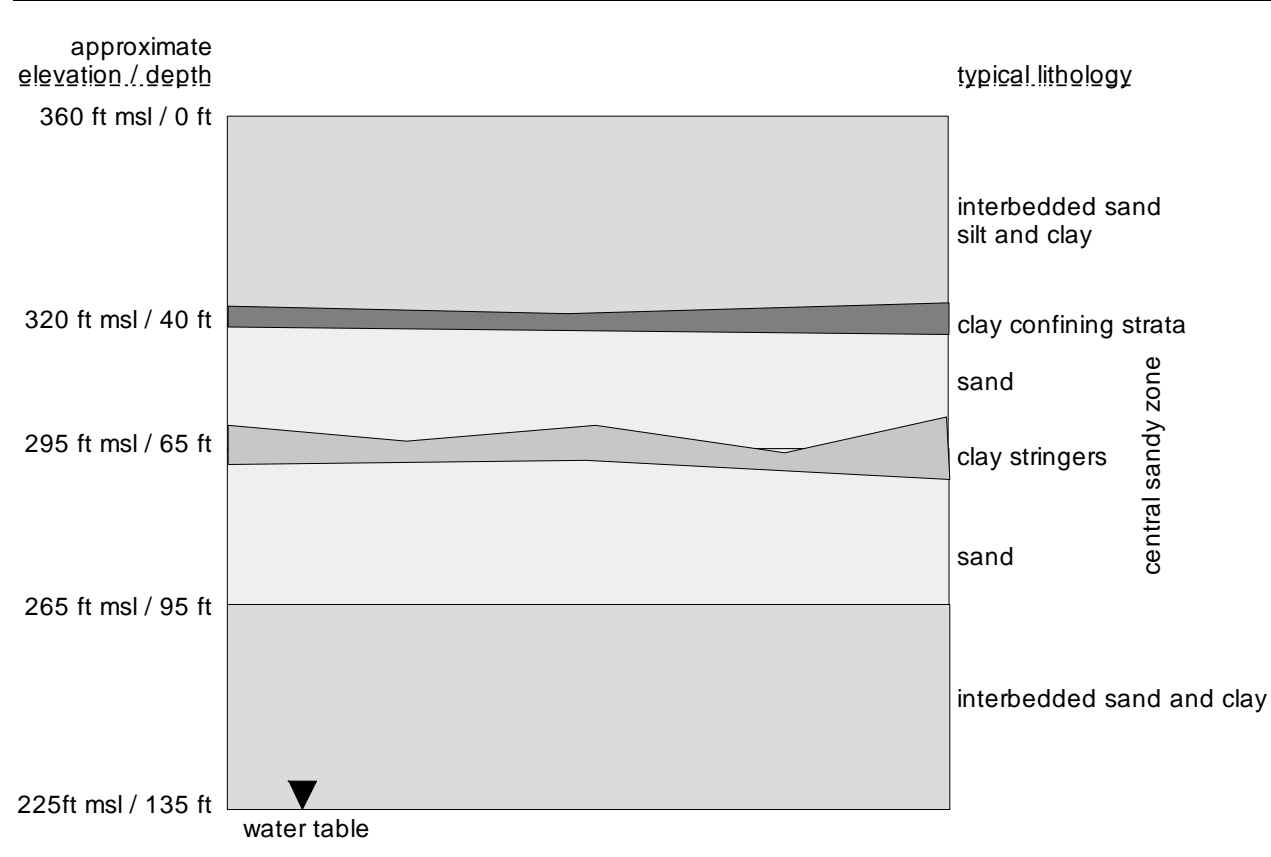

Figure 3 - Schematic of the A/M Area Vadose Zone 


\section{Methods}

\section{Existing Vadose Zone Monitoring Well Sampling}

Concentration measurements were made on 60 existing vadose zone monitoring points prior to determining cone penetrometer (CPT) characterization locations. These monitoring points consisted of a variety of well types with six multilevel sample points associated with SVE unit $782-5 \mathrm{M}, 19$ points associated with $782-7 \mathrm{M}$, and 35 points associated with $782-8 \mathrm{M}$. The well locations and concentrations are provided in Appendix A. Well vapor was sampled and analyzed in the field using an Innova Model 1312 photo-acoustic infrared gas analyzer (new model of the Bruel and Kjaer Model 1302). The instrument was calibrated to measure trichloroethylene (TCE), tetrachloroethylene (PCE), and carbon dioxide, $\left(\mathrm{CO}_{2}\right)$.

\section{CPT Characterization}

Twenty CPT characterization borings were completed in November of 2000 for soil gas and sediment sampling. A total of 282 sediment samples and 155 soil gas samples were collected.

\section{Sediment Sampling and Analysis}

The DOE CPT was used with the CPT wireline sampling system. Applied Research Associates (ARA, INC.) developed the CPT wireline system with funding from the Department of Energy's National Energy Technology Laboratory (NETL). The system was purchased and is one of the tools available on the DOE CPT truck. The wireline soil sampling tool uses 2 inch diameter rods with a removable core barrel with a locking mechanism that fits inside the push rods. The $1 \mathrm{ft}$ long by 1 inch internal diameter core barrel is deployed and retrieved using a wire tether. This new method provides a significant time and cost savings over traditional CPT soil sampling that requires the retrieval of the cone rods for each sample.

After the core is brought to the surface, an approximate 2 cubic centimeter (cc) plug sample was collected using a modified plastic syringe. The plug was transferred to a $22 \mathrm{ml}$ glass headspace vial and $5 \mathrm{ml}$ of nano-pure water was added. The vial was then sealed with a crimped Teflon-lined septum top for head-space analysis. Duplicate samples were collected at each depth and all samples were stored at $4^{\circ} \mathrm{C}$ until analyzed. In the field, the samples were described as sand, clay, or sand and clay mix.

The technique used to prepare and analyze soil samples for VOC analysis is a modified version of EPA Method 5021 which has been used successfully at the SRS since 1991. Each sample is weighed and then analyzed on the HP 5890 Series II gas chromatograph using an automated head space sampler at $70 \mathrm{C}$ for equivalent water concentrations. The GC is equipped with an electron capture and flame ionization detector connected in parallel. The column is a Supelco - VOCOLTM megabore borosilicate glass ( $60 \mathrm{~m} \times 0.76 \mathrm{~mm}$ ID $\times 1.5 \mu \mathrm{m}$ film thickness) specifically developed for volatile priority pollutants (EPA Methods 502, 602, and 8240). Mass 
soil concentrations $(\mathrm{ppb}, \mu \mathrm{g} / \mathrm{kg}$ ) are calculated based on an equal head space volume from $7.5 \mathrm{ml}$ of water standards and nominal $7 \mathrm{ml}$ of water/soil matrix and are corrected for the mass difference between the soil and water. The gas chromatograph is calibrated using purchased certified mixtures in methanol that are diluted in deionized water to specific concentrations. Two reagent blanks of pure deionized water are included to ensure the transfer lines and column are being adequately flushed of residual solvents. The standard concentrations used for each head space sample run are: $3,5,10,50,100,1,000$, and 10,000 ppb $(\mu \mathrm{g} / \mathrm{l})$. The samples were analyzed for vinyl chloride, Freon11, Freon-113, 1,1-dichloroethylene (1,1-DCE), trands-dichloroethylene (trans-DCE), cis-dichloroethylene (cis-DCE), chloroform, 1,1,1-Trichloroethane (TCA), carbon tetrachloride $\left(\mathrm{CCl}_{4}\right)$, trichloroethylene (TCE), and perchloroethylene (PCE). Approximately 25 percent of the duplicate samples were analyzed.

\section{Soil Gas Sampling and Analysis}

For soil gas sampling, the wireline push tip was withdrawn from the rods leaving the rods open at the bottom and soil gas was pumped directly through the rods using a dual head Teflon diaphragm pump. The use of the rods of the wire line tool for the collection of soil gas provides a significant amount of flow (up to $1.5 \mathrm{ft}^{3} /$ minute (cfm) with the pump used) compared to running tubing from a sample port in the cone tip. Soil gas samples were collected at depth intervals of about every $10 \mathrm{ft}$. The soil gas was analyzed in the field using an Innova Model 1312 photo-acoustic infrared gas analyzer (new model of the Bruel and Kjaer Model 1302). The instrument was calibrated to measure TCE, PCE, and carbon dioxide $\left(\mathrm{CO}_{2}\right)$. The results from the soil gas sampling are provided in Appendix C. 


\section{Results and Discussion}

\section{Soil Vapor Extraction Units}

The three SVE units have removed approximately $91,500 \mathrm{lbs}$ of contamination from the vadose zone along the process sewer line. The SVE has primarily addressed the central sandy unit (320 to $265 \mathrm{ft} \mathrm{msl}$ ) with contaminant removal from the lower permeability units dictated by mass transfer limitations. The contaminant removal rates are decreasing exponentially as expected. Each of the units is discussed below and measures to address future remediation will be discussed in a later section.

\section{SVE Unit 782-5M}

SVE Unit $782-5 \mathrm{M}$ was connected to vertical wells MVE-5 through MVE-8 and has been removed due to low contaminant removal rates. This unit began operating in April 1995. The SVE wells are screened in the sand region below the $320 \mathrm{ft}$ clay down into the $265 \mathrm{ft}$ clay. Wells MVE- 5 and MVE- 6 were installed directly at the location of manholes from the old process sewer. Testing was done to evaluate the transient and sustained rebound extraction rates. Production rates from the unit are below the $40 \mathrm{lbs} /$ week. The unit was shut down for two 90 day periods and restarted with little production rebound. As seen in Figure 4, after the first restart, production rates increased but then decreased within 3 months. During the second restart, production did not rebound significantly and production rates dropped to less than 30 lbs/week within one week. Remediation using passive soil vapor extraction (barometric pumping) is proposed based on the diminishing returns of the active system (Amari, 2000).

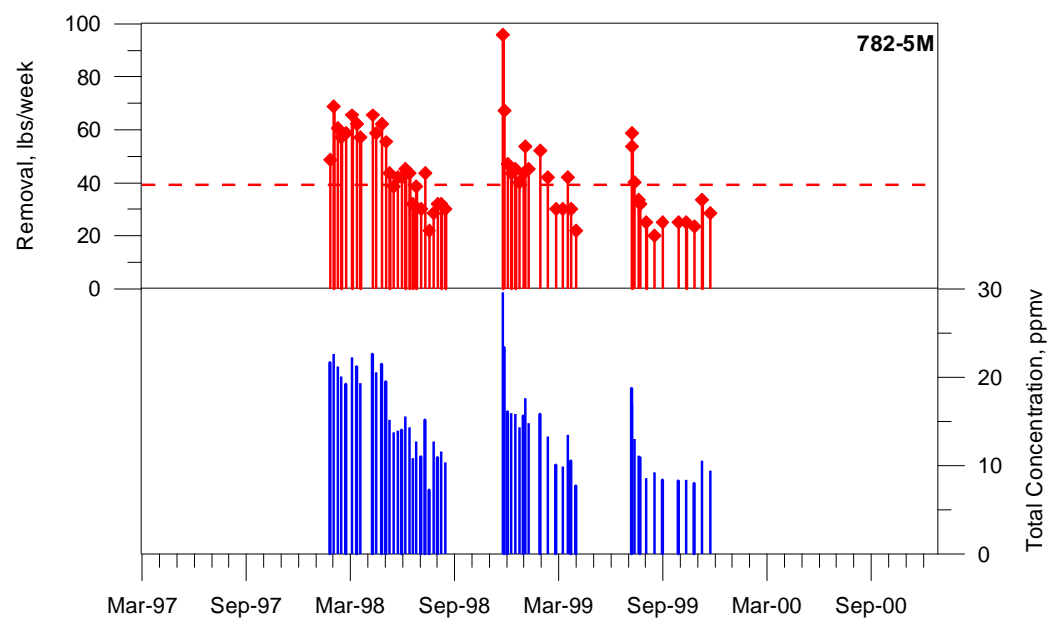

Figure 4 - Concentration and Removal Rates for SVE Unit 782-5M 


\section{SVE Unit 782-7M}

SVE Unit 782-7M is connected to horizontal well AMH-2 and is currently removing approximately $25 \mathrm{lbs} /$ week. Well AMH-2 is screened across the $300 \mathrm{ft}$ clay from approximately $310 \mathrm{ft} \mathrm{msl}$ to $285 \mathrm{ft} \mathrm{msl}$. The unit has been in operation since 1997 with flow rates of approximately $320 \mathrm{cfm}$. AMH-2 was used for the in-situ air stripping and bioremediation demonstrations prior to operations with SVE Unit 782$7 \mathrm{M}$. The concentration and removal rates are shown in Figure 5.

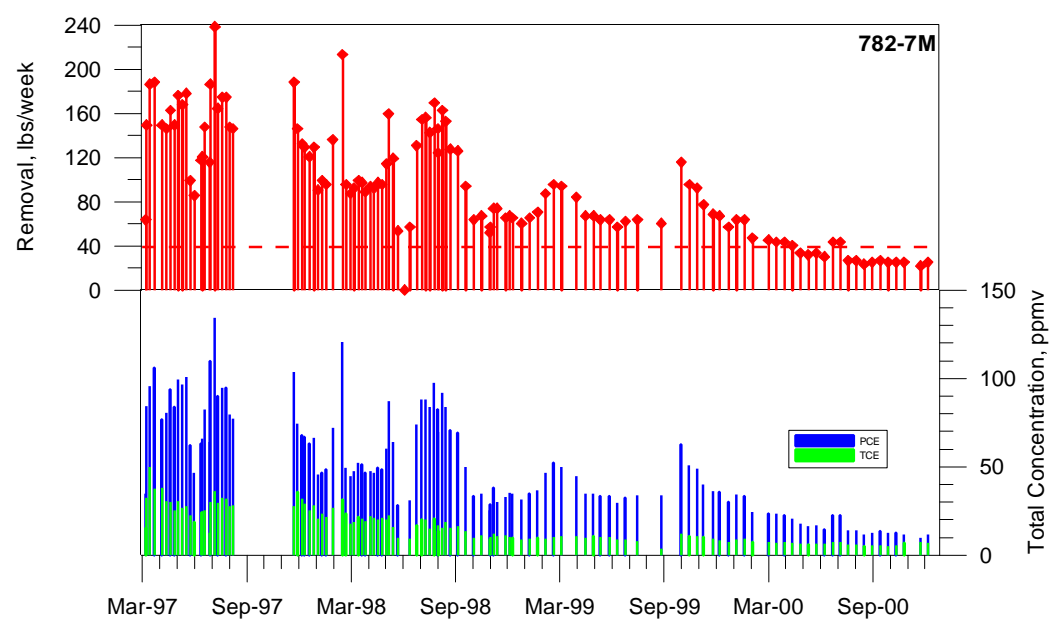

Figure 5 - Concentration and Removal Rates for SVE Unit 782-7M

\section{SVE Unit 782-8M}

SVE Unit $782-8 \mathrm{M}$ is connected to horizontal wells $\mathrm{AMH}-4$ and $\mathrm{AMH}-5$ and is not currently operating due to low contaminant removal rates. Well AMH-4 was installed as a demonstration of horizontal drilling technologies and was placed in the deep vadose zone at approximately $100 \mathrm{ft}$ depth. The screen zone is approximately $150 \mathrm{ft}$ long and is screened primarily in fine grain materials. This well was known to have some clogging and the areas of open screen zones are not known. Well AMH-5 was used for the radio frequency heating demonstration and is screened in a clay layer at approximately $40 \mathrm{ft}$ depth. The screen length is approximately $300 \mathrm{ft}$. Portions of the screen zone may be melted and the radio frequency heating antennae remains in the well. The unit produced approximately $160 \mathrm{cfm}$ of flow from the two wells. Lower flow rates were expected since both wells are screened in the fine grain sediments. Two attempts were made to redevelop the wells to increase flow rates. The operation history for the unit is provided in Table 1 and the contaminant removal rates and vapor concentrations are shown in Figure 6 . The remediation in this area is limited by well performance. Additional wells will be proposed to accelerate the remediation in this area of the process sewer line. 
Table 1- SVE Unit 782-8M Operation History

\begin{tabular}{|c|l|}
\hline Dates & Activity \\
\hline $4 / 97$ & 8 M starts operations \\
\hline $5 / 97$ & Wells Clogged \\
\hline $6 / 97$ & Wells Redeveloped and 8M restarted \\
\hline $9 / 24 / 97$ & Last time the loading to the unit was above $40 \mathrm{lb} /$ week \\
\hline $2 / 27 / 98$ & $8 \mathrm{M}$ shut down due to low contaminant loading (below $40 \mathrm{lb} /$ week) \\
\hline $5 / 98-6 / 98$ & Wells Redeveloped \\
\hline $6 / 11 / 98$ & $8 \mathrm{M}$ restarted \\
\hline $6 / 11 / 98-7 / 14 / 98$ & Mechanical Problems \\
\hline $7 / 14 / 98$ & $8 \mathrm{M}$ restarted \\
\hline $8 / 20 / 98$ & $8 \mathrm{M}$ shut down due to low contaminant loading (below $40 \mathrm{lb} /$ week) \\
\hline $11 / 24 / 98$ & $8 \mathrm{M}$ restarted \\
\hline $12 / 17 / 98$ & $8 \mathrm{M}$ shut down due to low contaminant loading (below $40 \mathrm{lb} /$ week) \\
\hline
\end{tabular}

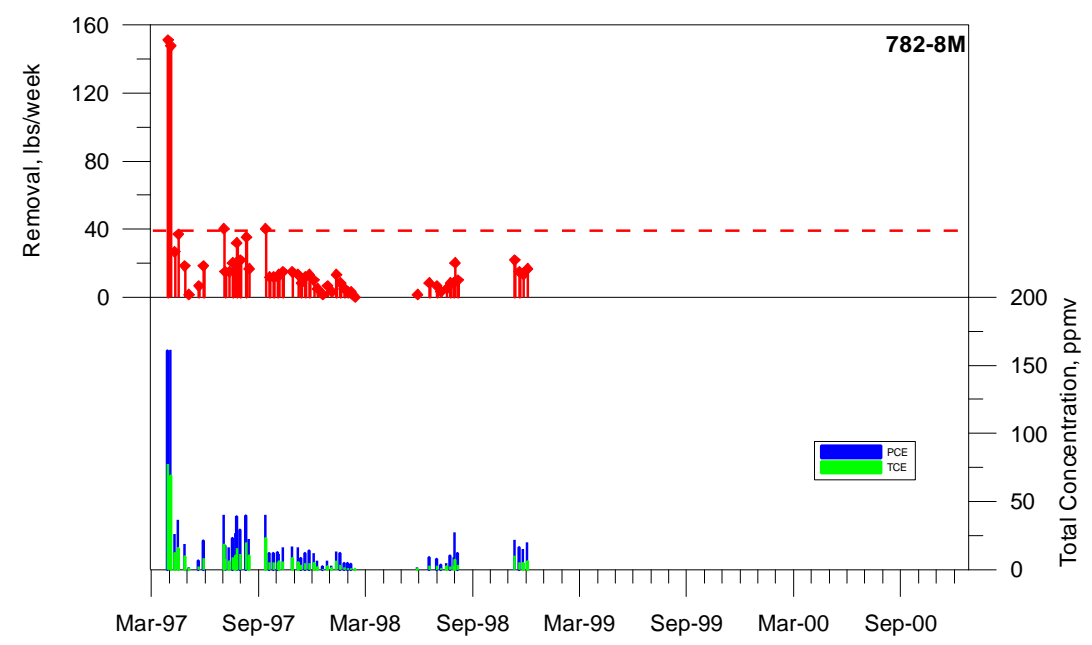

Figure 6 - Concentration and Removal Rates for SVE Unit 782-8M 


\section{Contaminant Profiles}

The figures in this section illustrate the pre-remediation and current soil gas and sediment concentrations. Plan views showing the initial and current maximum sediment concentration (total of PCE and TCE) for each boring are provided in Figures 7 and 8 . Initial sediment concentrations in the northern section of the study were not available. The figures show that contamination greater than $1,000 \mathrm{ug} / \mathrm{kg}$ still remains along the process sewer line. These higher concentrations correspond to the fine grain units above and below the central sandy unit that are not readily addressed by SVE.

The initial and current soil gas and sediment profiles for both TCE and PCE are depicted in Figures 9-12. These figures are three-dimensional images with a horizontal view from the southeast. See the three-dimensional image in Figure 2 for spatial reference. The extraction wells are shown in white. The screen sections of the directional wells are along the horizontal sections of the wells. For the vertical wells, just the screen section is shown. The black horizontal lines correspond to the lithology schematic in Figure 3 (Parker et al., 1999). The background covers the vertical extent of the vadose zone and shows the percent clay increasing from yellow to orange. The two deep horizontal wells extend below the water table; this extension is not shown. The data used for these images are provided in the appendices. See Table 2 for a list and description of the appendices.

Table 2 - Tabulated Data Descriptions

\begin{tabular}{|l|l|l|}
\hline Appendix & Title & Description \\
\hline A & $\begin{array}{l}\text { Vapor Concentrations } \\
\text { of Existing Wells }\end{array}$ & $\begin{array}{l}\text { Initial and current concentration measurements } \\
\text { of } 60 \text { existing vadose zone monitoring wells }\end{array}$ \\
\hline B & $\begin{array}{l}\text { Current Sediment } \\
\text { Concentrations }\end{array}$ & $\begin{array}{l}\text { Current PCE and TCE sediment concentrations } \\
\text { with sediment type CPT refusal depths }\end{array}$ \\
\hline C & $\begin{array}{l}\text { Current CPT Soil Gas } \\
\text { Concentrations }\end{array}$ & $\begin{array}{l}\text { Current PCE, TCE and } \mathrm{CO}_{2} \text { soil gas } \\
\text { concentrations from CPT characterization }\end{array}$ \\
\hline D & $\begin{array}{l}\text { Initial Sediment } \\
\text { Concentrations }\end{array}$ & $\begin{array}{l}\text { Initial PCE and TCE sediment concentrations, } \\
\text { only around SVE Units 7M and 8M }\end{array}$ \\
\hline E & $\begin{array}{l}\text { Boring and Well } \\
\text { Coordinates }\end{array}$ & $\begin{array}{l}\text { SRS coordinates, elevations, and screen zones } \\
\text { where applicable }\end{array}$ \\
\hline
\end{tabular}


The following results can be drawn from the data and these images:

- A significant amount of contamination has been removed from the vadose zone.

- DNAPL does not exist in the zones sampled $(<200,000 \mu \mathrm{g} / \mathrm{kg}$ TCE and $<50,000$ $\mu \mathrm{g} / \mathrm{kg}$ PCE) (Cohen and Mercer, 1993).

- Residual contamination remains in the fine grain zones, which are not directly addressed by SVE (above and below the central sandy unit).

- Low permeability zones, perched water and cemented zones in the lower fine grain zone (270 foot clay) drastically reduce the effectiveness of SVE from approximately $100 \mathrm{ft}$ down to the water table.

- The remediation between wells MVE-7 and AMH-2 is not being addressed well by the current SVE systems.

- The horizontal wells associated with SVE Unit 782-8M have not adequately addressed the vadose zone contamination. 


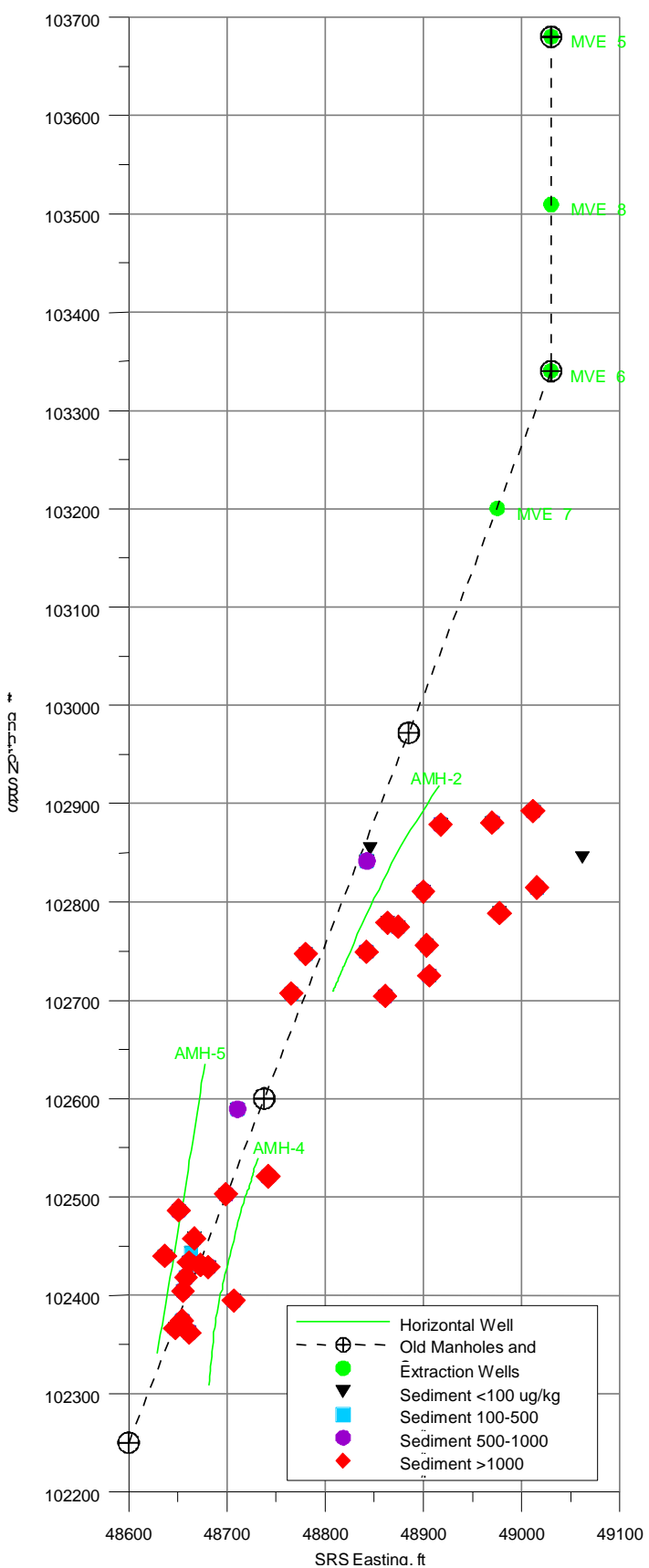

Figure 7 - Initial Maximum Total Sediment Concentration 


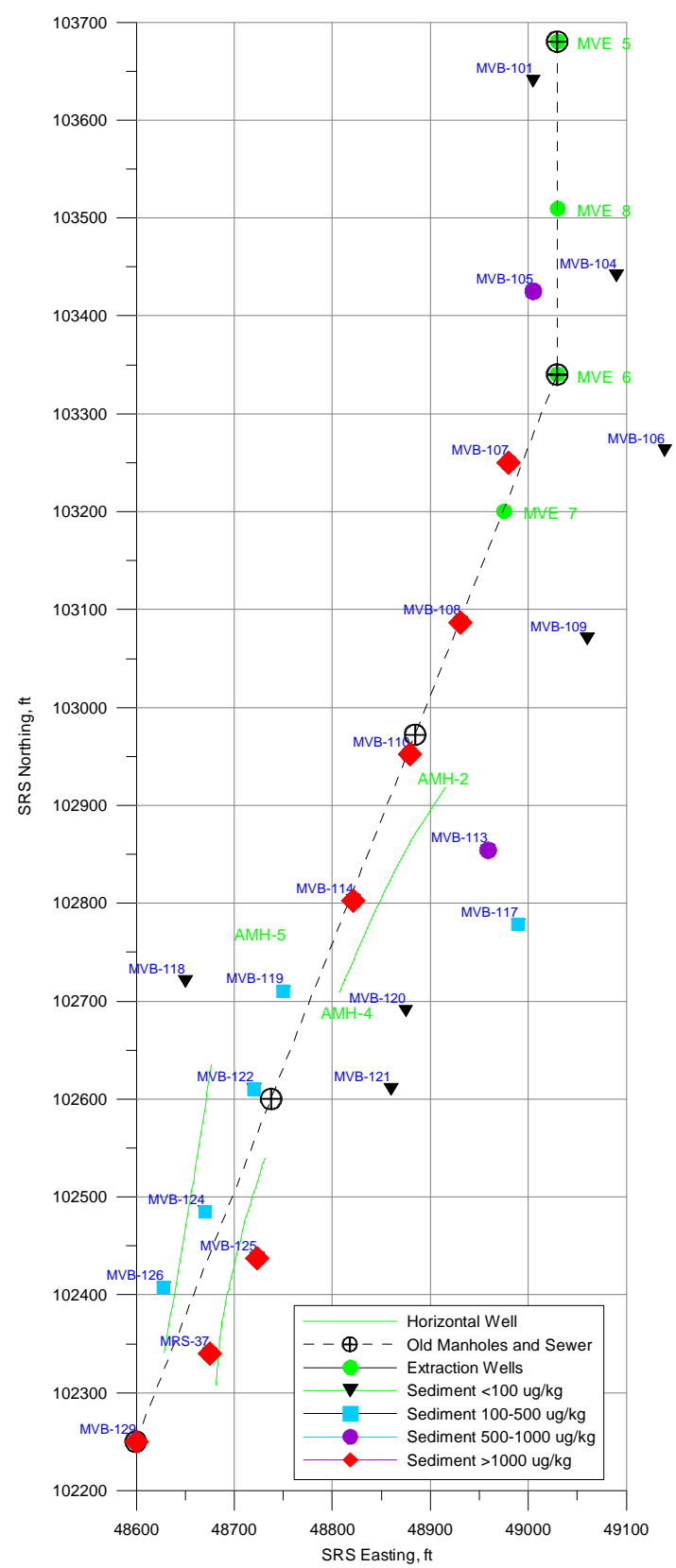

Figure 8 - Current Maximum Total Sediment Concentration 

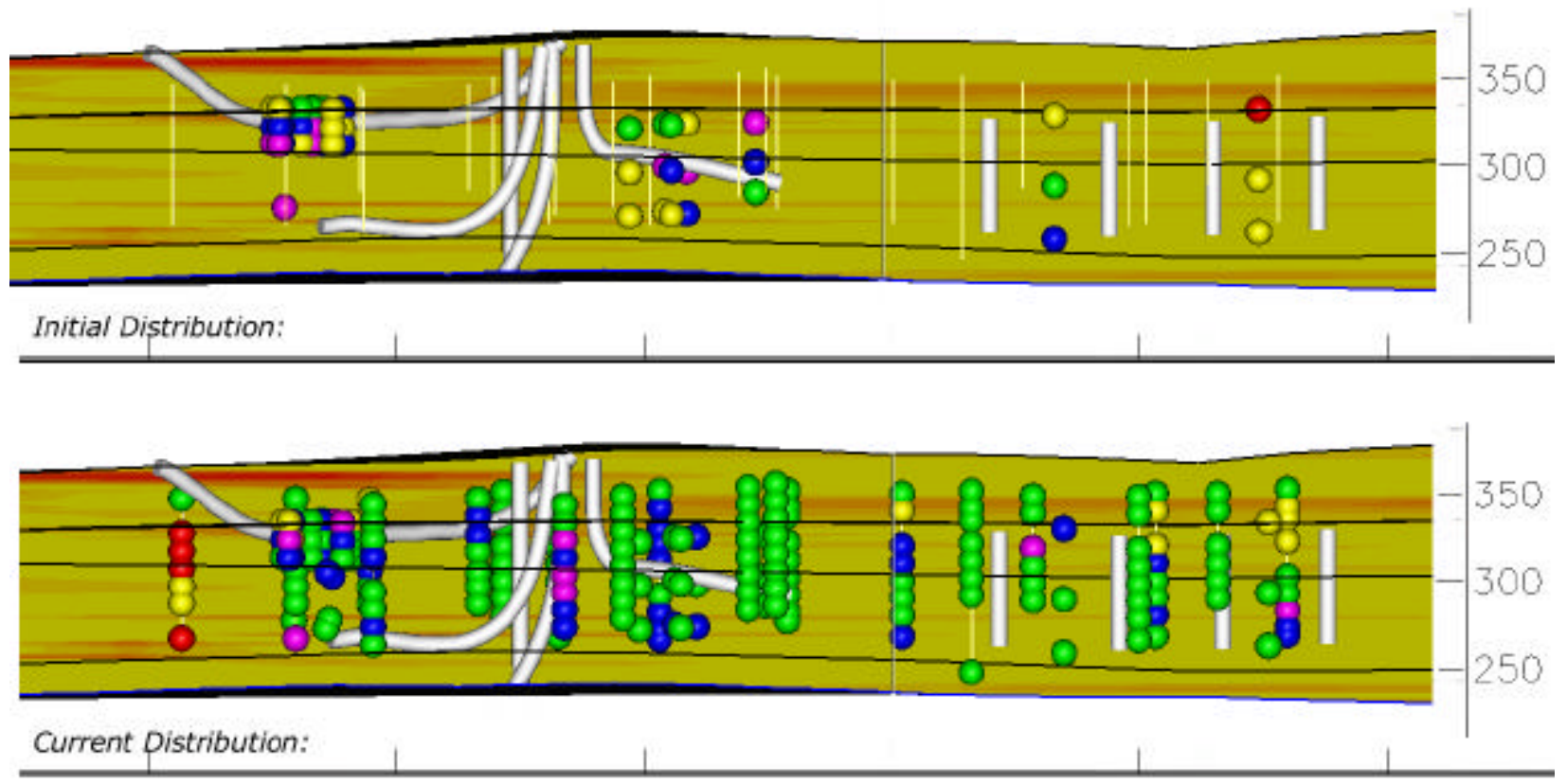

$-50 x$

$60-90 \%$

$70-808$

$60-70 x$

$50-60 x$

\$0-50x

$30-40 x$

20-30x

$<10 x$

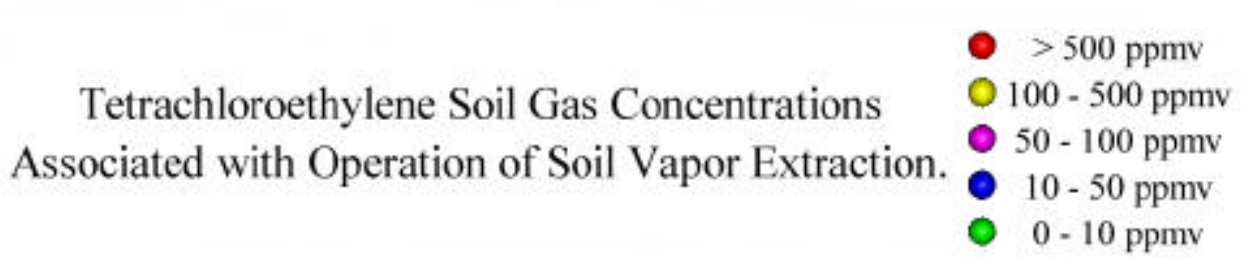

Figure 9 - PCE Soil Gas Concentration 

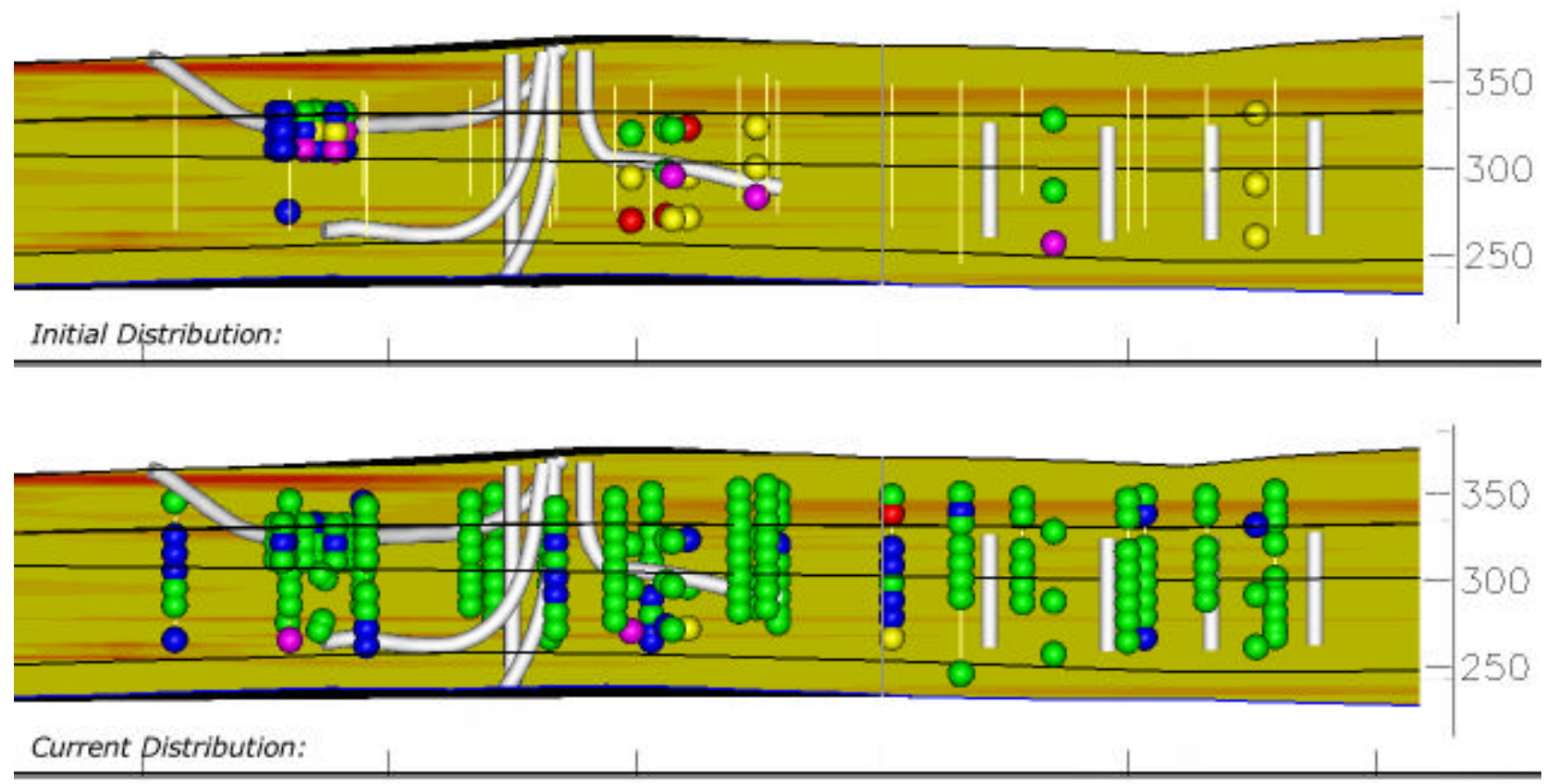

$\rightarrow 30 x$

$90-80 x$

$70-80 x$

$60-708$

50-60x

40-50x

$30-40 x$

$20-30 x$

$20-30 \%$

$<10 x$
Trichloroethylene Soil Gas Concentrations
Associated with Operation of Soil Vapor Extration.
- $>500 \mathrm{ppmv}$
O $100-500 \mathrm{ppmv}$
- $50-100 \mathrm{ppmv}$
- $10-50 \mathrm{ppmv}$
- $0-10 \mathrm{ppmv}$

Figure 10 - TCE Soil Gas Concentration 

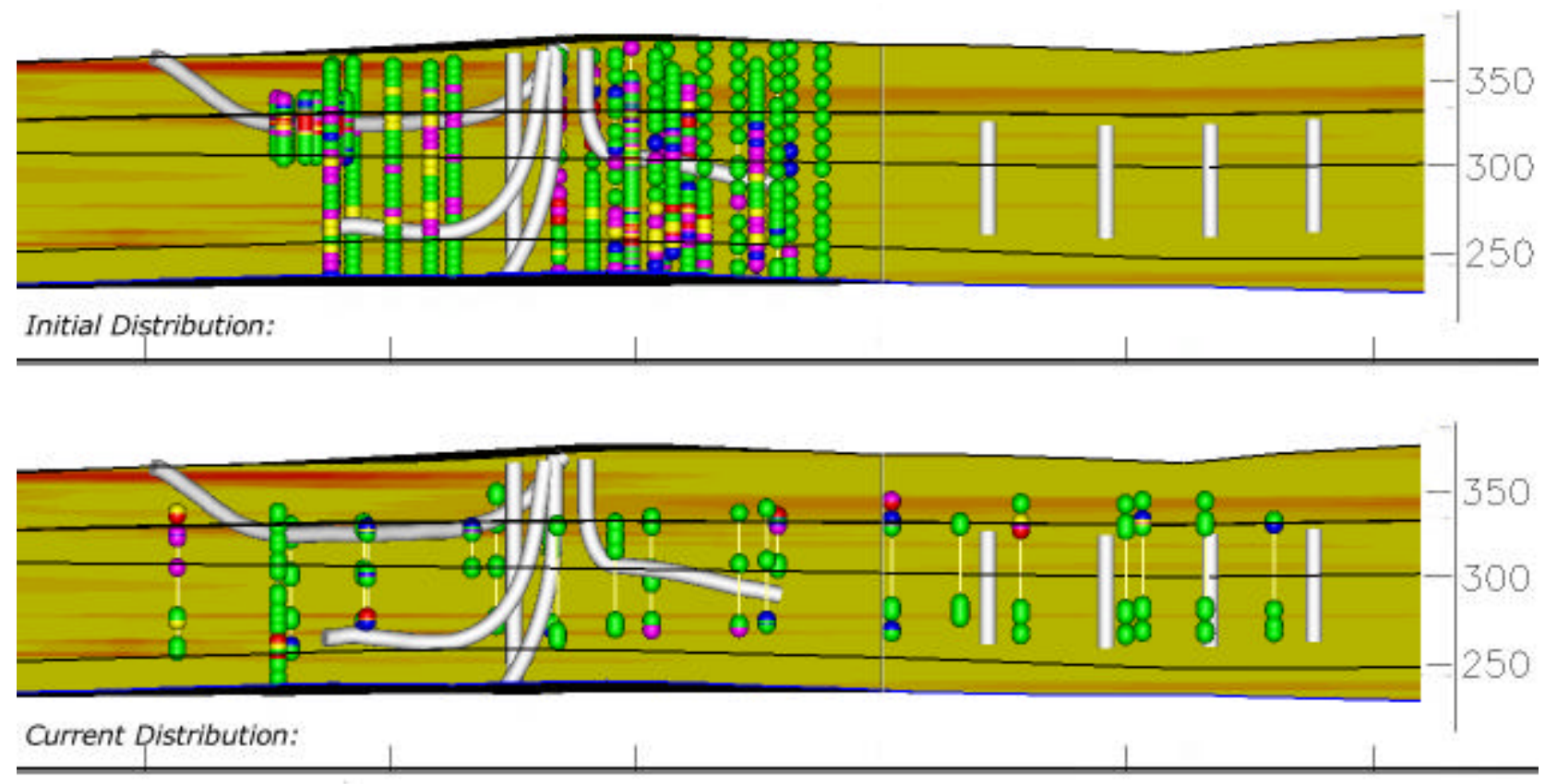

$>90 x$

80-90x

$70-80 x$

$60-70 x$

50-60x

40-50x

$30-40 x$

$20-50 x$

to- $-20 x$

$<10 x$

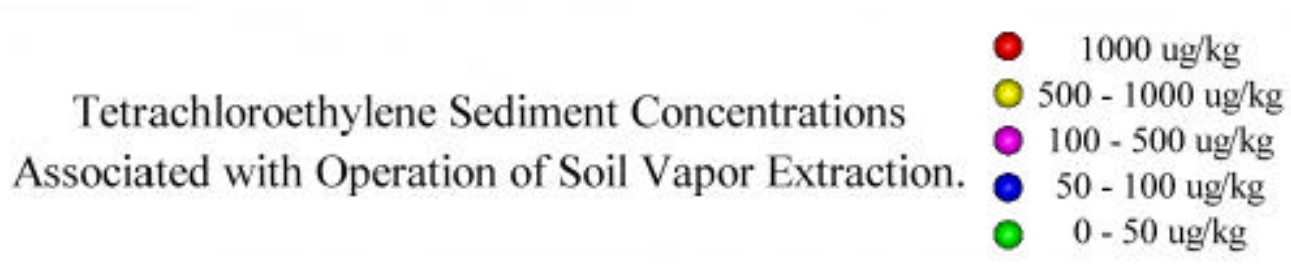

Figure 11- PCE Sediment Concentration 

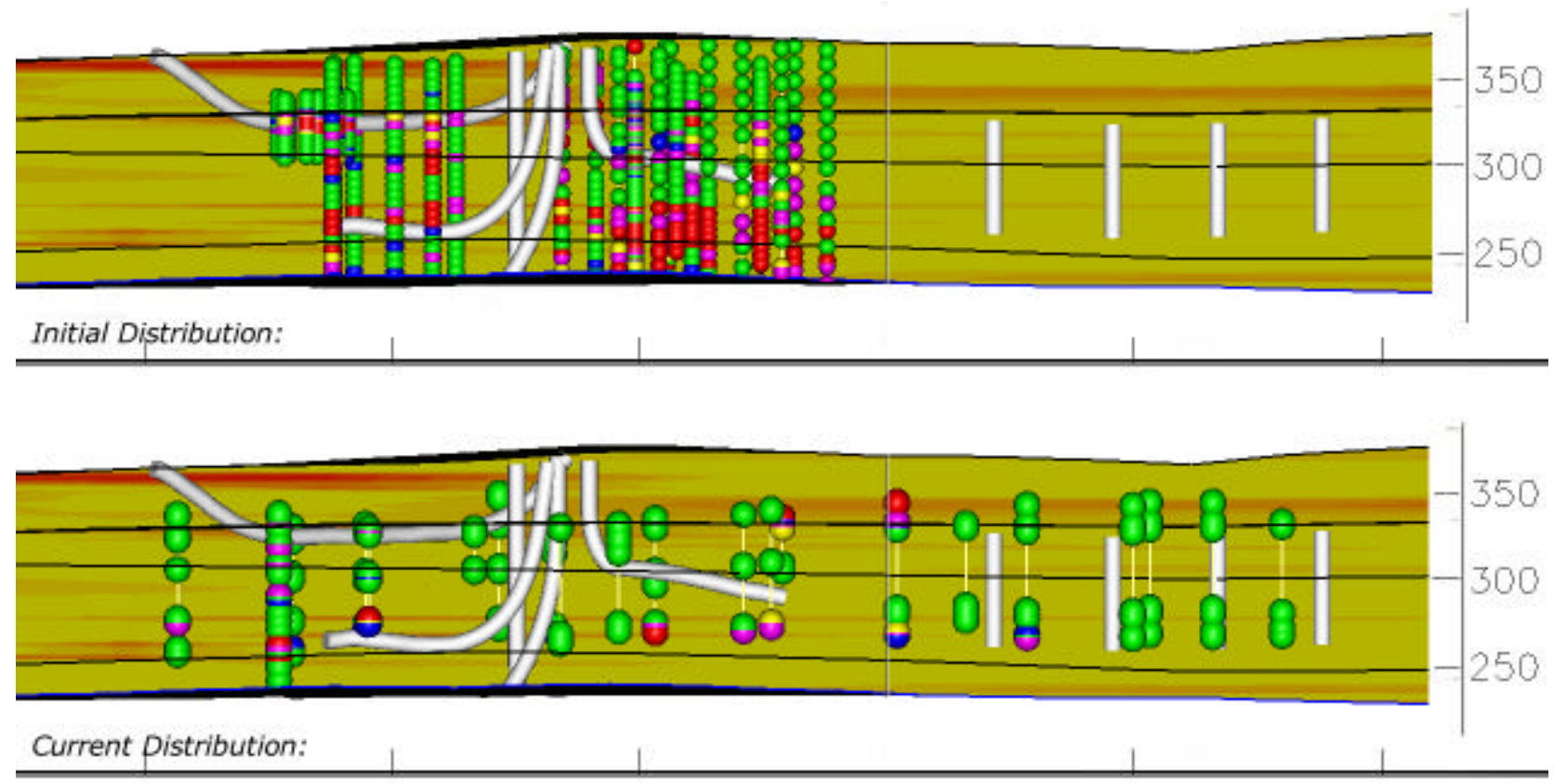

$>90 x$

$80-90 x$

$70-80 x$

$60-70 x$

50-60X

40-50x

$30-40 x$

$20-50 x$

$10-20 x$

$<10 x$

Trichloroethylene Sediment Concentrations

Associated with Operation of Soil Vapor Extraction.
- $1000 \mathrm{ug} / \mathrm{kg}$

$500-1000 \mathrm{ug} / \mathrm{kg}$

- $100-500 \mathrm{ug} / \mathrm{kg}$

- $50-100 \mathrm{ug} / \mathrm{kg}$

○ $0-50 \mathrm{ug} / \mathrm{kg}$

Figure 12 - TCE Sediment Concentration 


\section{Recommendations}

The recommendations will be addressed by current SVE Unit name and zone as described in Figure 1 . In general, the recommendations address residual contamination that remains in the fine grain sediments where remediation is regulated by mass transfer rates.

\section{Unit 782-5M: Transfer to Passive Soil Vapor Extraction}

Use wells MVE- 5 through MVE-8 for passive soil vapor extraction (PSVE) wells with BaroBallTM check valves. These wells correspond to SVE Unit 782-5M that has low contaminant removal rates. PSVE will address the current contaminant profile along the northern section of the study area. When the coarser sediments have been remediated, barometric pumping has proven to remove VOC contamination above the rate of release from the fine grained sediments at SRS. This trend has been observed as decreasing soil gas concentrations and plume size at both the Miscellaneous Chemical Basin and MetLab PSVE remediation sites (Riha et al., 1999a; Riha et al., 1999b; Riha et al., 2000)

Two flow measurements were made on well MVE-5 during January 2000 . The vapor flows were measured at 15 and $50 \mathrm{cfm}$ and are significantly higher than those measured from the two inch direct push wells at the current SRS PSVE remediation sites. The average vapor flow from the two inch direct push wells is approximately 2 $\mathrm{cfm}$. PSVE well vapor flows are a function of well size, screen length, screen zone, gravel pack, and differential pressure magnitude dictated by impermeable zones and barometric pressure fluctuations(Rossabi, 1999). The 4 inch diameter wells will provide a significant amount of flow. A discussion of barometric pumping flow and mass removal rates is provided in Appendix $\mathrm{F}$.

Periodic vapor sampling from MVE-5 through MVE-8 and MVC-4 and MVC-5 monitoring points is proposed to verify removal of contamination above the rate of release from the fine grain sediments. This will be demonstrated by a decrease in concentration over time.

The remediation between MVE-7 and AMH-2 is not being addressed adequately by the current remediation systems. Two 4 inch diameter wells are proposed near MVB-108 and near MVB-110 for use as PSVE wells. The wells will be installed with screens that address the upper and lower fine grain zones as well as the central sandy zone. These wells will be included in the PSVE monitoring plan that addresses the $5 \mathrm{M}$ unit.

\section{Unit 782-7M: Passive Soil Vapor Extraction Treatability Study}

Due to decreasing removal rates from $782-7 \mathrm{M}$, initiate a barometric pumping treatability study using the current $\mathrm{AMH}-2$ horizontal well and four existing vertical wells. SVE Unit $782-7 \mathrm{M}$ and well $\mathrm{AMH}-2$ are currently addressing the central sandy zone. The vertical wells are screened across the upper and lower fine grain zones where the contamination remains. Wells MHV-6 and MHV-7 are screened 
continuously from $10 \mathrm{ft}$ down to approximately 115 below the surface. Wells MHV-8 and MHV-9 have short multiple screens. Well construction details are provided in Table 3 and the wells are shown on the map in Figure 13. Based on the previous PSVE studies, these wells should adequately address the contaminant removal at the release rates expected from the fine grain zones.

A one year treatability study is proposed to determine the effectiveness of remediation using PSVE in this area. Monthly vapor sampling from the extraction wells and monitoring of selected monitoring points (MHV-1 to MHV-5) is proposed to verify removal of contamination above the rate of release from the fine grain sediments. This will be demonstrated by a decrease in concentration over time. Average well flow rates will also be determined. At the end of the study, a comparison between the active and passive removal rates will be made and it will be determined if active extraction should be reinstated.

Table 3 - Existing Four-Inch Diameter Wells Near 782-7M

\begin{tabular}{|c|c|c|c|c|c|c|}
\hline Well ID & $\begin{array}{l}\text { SRS } \\
\text { North }\end{array}$ & SRS East & $\begin{array}{l}\text { Screen } \\
\text { Top } \\
\text { ft msl }\end{array}$ & $\begin{array}{l}\text { Screen } \\
\text { Bottom } \\
\mathrm{ft} \mathrm{msl}\end{array}$ & $\begin{array}{l}\text { Surface } \\
\mathrm{ft} \mathrm{msl}\end{array}$ & \begin{tabular}{|l} 
Effective \\
Depth \\
$\mathrm{ft}$
\end{tabular} \\
\hline MHV-6 & 102780.1 & 48891.5 & 358.8 & 253.8 & 368.1 & 114.6 \\
\hline MHV-7 & 102811.5 & 48878.1 & 358.2 & 253.2 & 368.2 & 117.1 \\
\hline \multirow[t]{7}{*}{ MHV-8 } & \multirow[t]{7}{*}{102610.3} & \multirow[t]{7}{*}{48846.3} & 323.4 & 321.1 & \multirow[t]{7}{*}{357.7} & \multirow[t]{7}{*}{117.4} \\
\hline & & & 303.8 & 301.5 & & \\
\hline & & & 296.5 & 294.2 & & \\
\hline & & & 285.2 & 282.9 & & \\
\hline & & & 268.9 & 266.7 & & \\
\hline & & & 254.7 & 252.4 & & \\
\hline & & & 243.5 & 241.2 & & \\
\hline \multirow[t]{8}{*}{ MHV-9 } & \multirow[t]{8}{*}{102830.1} & \multirow[t]{8}{*}{49046.7} & 343.5 & 341.2 & \multirow[t]{8}{*}{367.7} & \multirow[t]{8}{*}{123.6} \\
\hline & & & 318.8 & 316.5 & & \\
\hline & & & 303.5 & 301.2 & & \\
\hline & & & 292.2 & 290 & & \\
\hline & & & 281 & 278.7 & & \\
\hline & & & 268.7 & 266.4 & & \\
\hline & & & 255.3 & 253 & & \\
\hline & & & 248 & 245.7 & & \\
\hline
\end{tabular}

Unit 782-8M: Increase Flow to Well AMH-6 as an Interim Measure

The horizontal wells associated with SVE Unit 782-8M have not addressed the vadose zone contamination adequately. Initial SVE modeling work done by Clemson University shows the zone of capture from horizontal well AMH-6 under the basin reaches to SRS Northing 102500 (Falta, 2001). This means the subsurface in this area is under vacuum conditions and barometric pumping is not viable. This negative pressure condition has been measured in existing piezometers. 
Remediation in this area can be enhanced by increasing the subsurface flow along the sewer line to well AMH-6. Flow can be increased by installing additional vadose zone wells to allow surface air inflow that will sweep the contaminants through the more permeable zones to AMH-6.

These wells will also create a 'pressure barrier' between the basin and the northern sewer area and will allow the natural pressure fluctuations to occur which drives PSVE. The wells will be installed with screens that address the upper and lower fine grain zones as well as the central sandy zone. The three proposed wells are shown in Figure 13 near the 102500 SRS Northing line. Existing monitoring points will be used to evaluate the effectiveness of this strategy and wells can be used for active vapor extraction if necessary. This strategy is proposed as an interim measure contingent on the remedial design chosen for the M-Area Basin. An additional well is proposed near MVB-119 for PSVE to complete coverage of the process sewer line.

\section{Evaluate Alternative Remediation Techniques}

In general contamination remains in the upper fine grain, low permeability zone that is not directly addressed by active SVE. Heating and methods for increasing permeability should be evaluated to increase the remediation from these zones.

Low permeability zones, perched water and cemented zones are located in the lower fine grain zone, located approximately $100 \mathrm{ft}$ down to the water table. Additional characterization needs to be done in this zone to determine the contaminant distribution. A literature search can be done to find methods for remediating VOC plumes in these types of sediments and in the capillary fringe. 


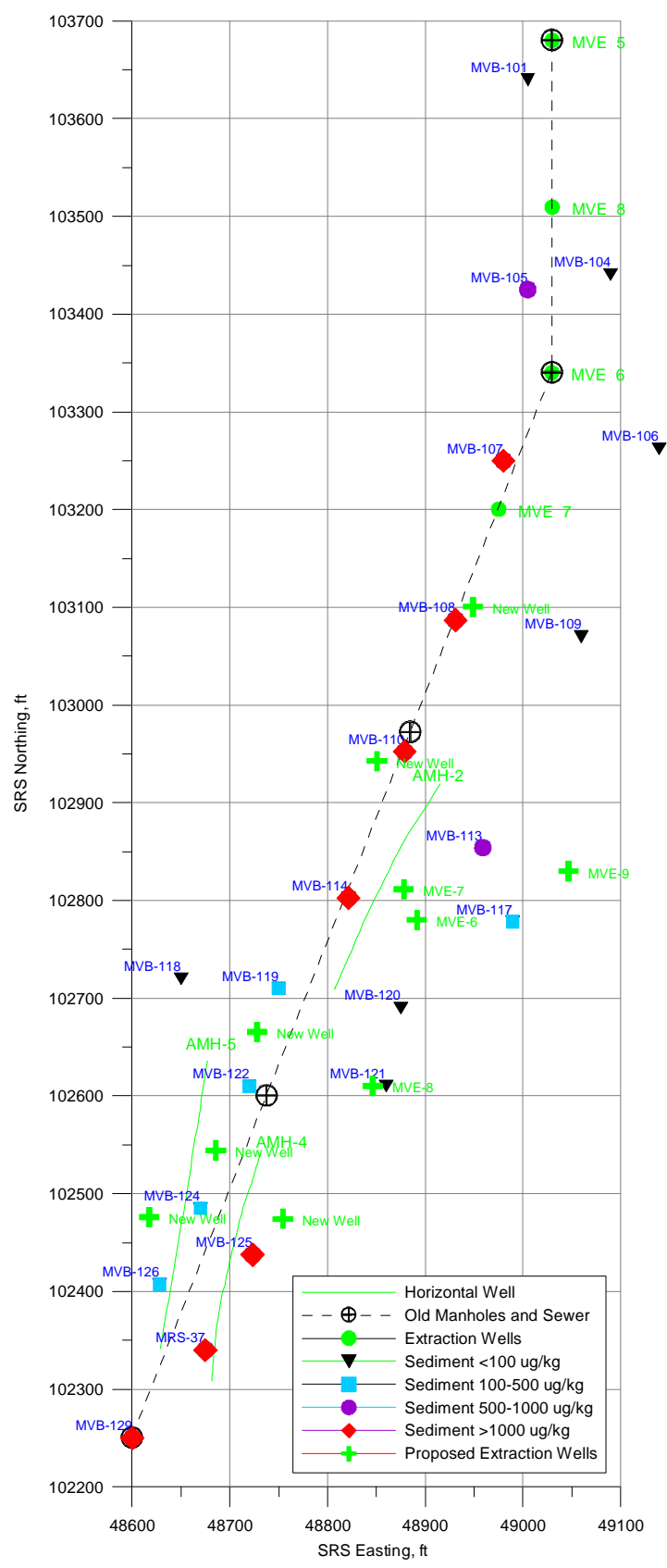

Figure 13 - Proposed Locations for New Vadose Zone Wells 


\section{References}

Amari, J. A. 2000. Shutdown of SVEU (Soil Vapor Extraction Unit) 782-5M and Use of Baroballs. Aiken, SC, Westinghouse Savannah River Company. ERD-991254.

Cohen, R. M. and J. W. Mercer 1993. DNAPL Site Evaluation, CRC Press.

Eddy, C. A., B. B. Looney, J. M. Dougherty, T. C. Hazen and D. S. Kaback. 1991. Characterization of the Geology, Geochemistry, Hydrology and Microbiology of the In-Situ Air Stripping Demonstration Site at the Savannah River Site (U). Aiken, South Carolina 29808, Westinghouse Savannah River Company. WSRCRD-91-0021.

Falta, R. W. 2001. Three-Dimensional Simulation of Multi-Phase Flow and Transport Processes for Evaluation of Soil Vapor Extraction Units 782-3m, -4m, and -7m, Clemson University. In press. SRS SCUREF project \#WEST047.

Jarosch, T. R., D. G. Jackson, B. B. Looney, K. M. Jerome, B. D. Riha, J. Rossabi, R. S. Van Pelt and J. J. Kupar. 1998. A/M Area Vadose Zone Monitoring Plan (U). Aiken, South Carolina 29808, Westinghouse Savannah River Company. WSRC-RP-98-00146.

Jarosch, T. R., D. G. Jackson, J. Rossabi, B. D. Riha, J. J. Kupar and R. S. Van Pelt. 1997. Extent of A/M Area Vadose Zone Contamination, Monitoring, and Corrective Action (U). Aiken, South Carolina 29808, Westinghouse Savannah River Company. WSRC-RP-97-0109.

Parker, W. H., A. D. Smits, M. K. Harris, D. G. Jackson and K. L. Hawkins. 1999. Baseline Mapping Study of the Steed Pond Aquifer and Vadose Zone Beneath A/M Area, Savannah River Site, Aiken, South Carolina (U). Aiken, South Carolina 29808, Westinghouse Savannah River Company. WSRC-TR-9900295.

Riha, B. D., J. Rossabi and W. K. Hyde. 1999a. Metallurgical Laboratory (MetLab) Treatability Study: An Analysis of Passive Soil Vapor Extractin Wells (PSVE), FY2000 Update. Aiken, SC, Westinghouse Savannah River Company. WSRCTR-99-00373.

Riha, B. D., J. Rossabi and W. K. Hyde. 1999b. Miscellaneous Chemical Basin Treatability Study: An Analysis of Passive Soil Vapor Extraction Wells (PSVE), FY1998 Update. Aiken, SC, Westinghouse Savannah River Company. WSRCTR-99-00058.

Riha, B. D., J. Rossabi and W. K. Hyde. 2000. Metallurgical Laboratory (MetLab) Treatability Study: An Analysis of Passive Soi Vapor Extraction Wells (PSVE), June 2000 Undate. Aiken, SC, Westinghouse Savannah River Company. WSRC-TR-2000-00182.

Rossabi, J. 1999. The Influence of Atmospheric Pressure Variations on Subsurface Soilgas and the Implications for Environmental Characterization and Remediation. Ph. D. Dissertation, Clemson University, Clemson, SC. 


\section{Appendix A - Vapor Concentrations of Existing Wells}

\begin{tabular}{|c|c|c|c|c|c|c|c|c|c|c|c|c|}
\hline $\begin{array}{l}\text { SVE } \\
\text { Unit }\end{array}$ & Well ID & $\begin{array}{l}\text { Oct-00 } \\
\text { PCE } \\
\text { ppmv }\end{array}$ & $\begin{array}{l}\text { Oct-00 } \\
\text { TCE } \\
\text { ppmv }\end{array}$ & $\begin{array}{l}\text { Oct-00 } \\
\mathrm{CO}_{2} \\
\text { ppmv }\end{array}$ & $\begin{array}{l}\text { Initial } \\
\text { PCE } \\
\text { ppmv }\end{array}$ & $\begin{array}{l}\text { Initial } \\
\text { TCE } \\
\text { ppmv }\end{array}$ & \begin{tabular}{|l} 
Initial \\
Sample \\
Date
\end{tabular} & \begin{tabular}{|l} 
SRS \\
Easting
\end{tabular} & $\begin{array}{l}\text { SRS } \\
\text { Northing }\end{array}$ & $\begin{array}{l}\text { Ground } \\
\text { Elevation } \\
\mathrm{ft}, \mathrm{msl}\end{array}$ & \begin{tabular}{|l|} 
Screen \\
Top \\
Elevation \\
ft, msl
\end{tabular} & $\begin{array}{l}\text { Screen } \\
\text { Bottom } \\
\text { Elevation } \\
\mathrm{ft}, \mathrm{msl}\end{array}$ \\
\hline \multirow[t]{7}{*}{$5-M$} & MVC-4A & 2.4 & 4.5 & 11500 & 363.8 & 362.3 & Nov-94 & 49018.8 & 103594.6 & 366.1 & 256.5 & 256.3 \\
\hline & MVC-4B & 4.1 & 2.1 & 3100 & 260.4 & 145.0 & Nov-94 & 49018.8 & 103594.6 & 366.1 & 286.9 & 286.7 \\
\hline & MVC-4C & 376.0 & 19.8 & 10900 & 1009.1 & 173.7 & Nov-94 & \begin{tabular}{|l|}
49018.8 \\
\end{tabular} & 103594.6 & 366.1 & 327.3 & 327.1 \\
\hline & MVC-5A & 0.9 & 5.3 & 9330 & 17.8 & 89.2 & Jun-97 & 49004.1 & $\mid 103276.8$ & 362.1 & 252.6 & 252.3 \\
\hline & MVC-5B ${ }^{a}$ & 0.0 & 0.5 & 377 & & & & 49004.1 & 103276.8 & 362.1 & 262.8 & 262.6 \\
\hline & MVC-5C & 0.5 & 1.1 & 5830 & 1.9 & 0.9 & Jun-97 & 49004.1 & 103276.8 & 362.1 & 283.1 & 282.9 \\
\hline & MVC-5D & 31.1 & 0.6 & 5960 & 191.2 & 1.1 & Sep-97 & 49004.1 & 103276.8 & 362.1 & 323.5 & 323.3 \\
\hline \multirow[t]{19}{*}{ 7-M } & MHV-1A & 9.3 & 54.6 & 12300 & 346.0 & 656.0 & Jul-90 & 48842 & 102749.3 & 365.6 & 270.6 & 265.6 \\
\hline & MHV-1B & 0.8 & 4.6 & 4910 & 233.0 & 173.0 & Jul-90 & 48842 & 102749.3 & 365.6 & 295.6 & 290.6 \\
\hline & $\mathrm{MHV}-1 \mathrm{C}^{\mathrm{a}}$ & 0.1 & 0.2 & 343 & 5.0 & 2.6 & Jul-90 & 48842 & 102749.3 & 365.6 & 321.6 & 315.6 \\
\hline & $\mathrm{MHV}-2 \mathrm{~A}$ & 3.6 & 5.5 & 13100 & 108.0 & 209.0 & Jul-90 & 48903.2 & 102755.9 & 366.4 & 271.4 & 266.4 \\
\hline & MHV-2B & 1.4 & 1.0 & 13900 & 40.0 & 70.5 & Jul-90 & 48903.2 & 102755.9 & 366.4 & 296.4 & 291.4 \\
\hline & MHV-2C & 0.6 & 0.6 & 13700 & 2.3 & 1.2 & Jul-90 & 48903.2 & 102755.9 & 366.4 & 322.4 & 317.4 \\
\hline & MHV-3A & 14.2 & 15.2 & 9370 & 225.0 & 532.0 & Jul-90 & 48874.1 & $\mid 102774.7$ & 368.2 & 273.2 & 268.2 \\
\hline & MHV-3B & 0.2 & 0.7 & 2670 & 59.8 & 5.0 & Jul-90 & 48874.1 & $\mid 102774.7$ & 368.2 & 298.8 & 293.8 \\
\hline & MHV-3C & 1.4 & 3.6 & 11700 & 0.5 & 0.2 & Jul-90 & 48874.1 & \begin{tabular}{|l|}
102774.7 \\
\end{tabular} & 368.2 & 323.2 & 318.2 \\
\hline & $\mathrm{MHV}-4 \mathrm{~A}$ & 20.1 & 134.0 & 10900 & 22.9 & 207.0 & Jul-90 & 48842.5 & 102841.7 & 366.1 & 272.1 & 267.1 \\
\hline & MHV-4B & 0.5 & 1.0 & 6920 & 57.2 & 352.0 & Jul-90 & 48842.5 & 102841.7 & 366.1 & 296.1 & 291.1 \\
\hline & MHV-4C & 42.0 & 42.9 & 12100 & 386.7 & 731.0 & Jul-90 & 48842.5 & 102841.7 & 366.1 & 323.6 & 318.6 \\
\hline & MHV-5A & 0.5 & 0.9 & 5160 & 9.1 & 61.3 & Jul-90 & 48917.5 & 102878.8 & 369.2 & 284.2 & 279.2 \\
\hline & MHV-5B & 0.7 & 1.1 & 9500 & 21.6 & 320.0 & Jul-90 & 48917.5 & 102878.8 & 369.2 & 301.2 & 296.2 \\
\hline & MHV-5C & 3.5 & 6.2 & 20400 & 89.6 & 268.0 & Jul-90 & 48917.5 & 102878.8 & 369.2 & 324.2 & 319.2 \\
\hline & MHV-6 & 0.0 & 0.3 & 11900 & & & & 48891.5 & 102780.1 & 368.1 & 358.8 & 253.8 \\
\hline & MHV-7 & 0.0 & 0.5 & 5590 & & & & 48878.1 & 102811.5 & 368.2 & 358.2 & 253.2 \\
\hline & $\mathrm{MHV}-8^{\mathrm{a}, \mathrm{b}}$ & 0.5 & 1.3 & 1860 & & & & 48846.3 & 102610.3 & 357.7 & 323.4 & 241.2 \\
\hline & MHV-9 ${ }^{b}$ & 0.3 & 0.2 & 20200 & & & & 49046.7 & 102830.1 & 367.7 & 343.5 & 245.7 \\
\hline
\end{tabular}

a - low $\mathrm{CO}_{2}$ usually indicates poor sample recovery

$\mathrm{b}$ - wells have multiple screen zones

$c-$ bottom of wells were measured with probable screen lengths of $5-10 \mathrm{ft}$ 


\begin{tabular}{|c|c|c|c|c|c|c|c|c|c|c|c|c|}
\hline $\begin{array}{l}\text { SVE } \\
\text { Unit }\end{array}$ & Well ID & $\begin{array}{l}\text { Oct-00 } \\
\text { PCE } \\
\text { ppmv }\end{array}$ & $\begin{array}{l}\text { Oct-00 } \\
\text { TCE } \\
\text { ppmv }\end{array}$ & $\begin{array}{l}\text { Oct-00 } \\
\mathrm{CO}_{2} \\
\mathrm{ppm}\end{array}$ & $\begin{array}{l}\text { Initial } \\
\text { PCE } \\
\text { ppmv }\end{array}$ & $\begin{array}{l}\text { Initial } \\
\text { TCE } \\
\text { ppmv }\end{array}$ & $\begin{array}{l}\text { Initial } \\
\text { Sample } \\
\text { Date }\end{array}$ & $\begin{array}{l}\text { SRS } \\
\text { Easting }\end{array}$ & $\begin{array}{l}\text { SRS } \\
\text { Northing }\end{array}$ & $\begin{array}{l}\text { Ground } \\
\text { Elevation } \\
\mathrm{ft}, \mathrm{msl}\end{array}$ & \begin{tabular}{|l|} 
Screen \\
Top \\
Elevation \\
$\mathrm{ft}, \mathrm{msl}$
\end{tabular} & $\begin{array}{l}\text { Screen } \\
\text { Bottom } \\
\text { Elevation } \\
\mathrm{ft}, \mathrm{msl} \\
\end{array}$ \\
\hline \multirow[t]{35}{*}{ 8-M } & MHV-20A & 13.6 & 0.8 & 9950 & 63.4 & 33.0 & Feb-93 & 48658.3 & 102418.4 & 359.6 & 311.6 & 306.7 \\
\hline & MHV-20B & 2.5 & 1.1 & 16400 & 88.0 & 132.5 & Feb-93 & 48658.3 & 102418.4 & 359.6 & 321.6 & 316.7 \\
\hline & MHV-20C & 16.9 & 16.1 & 19300 & 0.0 & 0.0 & Feb-93 & 48658.3 & 102418.4 & 359.6 & 331.6 & 326.7 \\
\hline & MHV-21A & 5.6 & 4.2 & 20800 & 23.8 & 16.3 & Feb-93 & 48661.5 & 102433.6 & 359.7 & 311.7 & 306.8 \\
\hline & MHV-21B & 52.7 & 52.6 & 18400 & 25.0 & 38.2 & Feb-93 & 48661.5 & 102433.6 & 359.7 & 321.7 & 316.8 \\
\hline & MHV-21C & 17.6 & 0.0 & 21900 & 0.0 & 0.0 & Feb-93 & 48661.5 & 102433.6 & 359.7 & 331.7 & 326.8 \\
\hline & MHV-22A & 1.0 & 1.3 & 18500 & 74.7 & 46.1 & Feb-93 & 48663.8 & 102443.5 & 359.7 & 311.7 & 306.8 \\
\hline & MHV-22B & 17.6 & 4.3 & 6720 & 143.9 & 66.9 & Feb-93 & 48663.8 & 102433.5 & 359.7 & 321.7 & 316.8 \\
\hline & MHV-22C & 68.7 & 0.9 & 22600 & 0.0 & 0.0 & Feb-93 & 48663.8 & 102433.5 & 359.7 & 331.7 & 326.8 \\
\hline & MHV-23A & 3.6 & 2.6 & 19500 & 37.8 & 26.6 & Feb-93 & 48666.9 & 102458.0 & 359.7 & 311.7 & 306.8 \\
\hline & $M H V-23 B^{a}$ & 0.2 & 0.7 & 487 & 155.0 & 77.6 & Feb-93 & 48666.9 & 102458.0 & 359.7 & 321.7 & 316.8 \\
\hline & MHV-23C & 113.0 & 0.5 & 24500 & 30.5 & 3.7 & Feb-93 & 48666.9 & 102458.0 & 359.7 & 331.7 & 326.8 \\
\hline & MHV-24A ${ }^{a}$ & 0.2 & 0.3 & 1100 & 25.5 & 13.6 & Feb-93 & 48636.9 & 102439.8 & 360.5 & 312.5 & 307.6 \\
\hline & MHV-24B & 13.9 & 9.5 & 19900 & 30.0 & 26.9 & Feb-93 & 48636.9 & 102439.8 & 360.5 & 322.5 & 317.6 \\
\hline & MHV-24C & 0.3 & 0.2 & 20200 & 1.1 & 0.0 & Feb-93 & 48636.9 & 102439.8 & 360.5 & 332.5 & 327.6 \\
\hline & MHV-25A & 1.4 & 0.9 & 12400 & 169.8 & 78.1 & Feb-93 & 48681 & 102428.9 & 359.1 & 311.1 & 306.2 \\
\hline & MHV-25B & 30.2 & 19.7 & 21400 & 259.1 & 370.9 & Feb-93 & 48681 & 102428.9 & 359.1 & 321.1 & 316.2 \\
\hline & MHV-25C & 83.8 & 0.5 & 21800 & 135.8 & 12.8 & Feb-93 & 48681 & 102428.9 & 359.1 & 331.1 & 326.2 \\
\hline & $\mathrm{MHV}-26 \mathrm{~A}$ & 1.0 & 0.4 & 6050 & 150.4 & 73.9 & Feb-93 & 48655.3 & 102404.2 & 359.8 & 311.8 & 306.9 \\
\hline & MHV-26B & 6.9 & 4.7 & 13600 & 32.7 & 18.3 & Feb-93 & 48655.3 & 102404.2 & 359.8 & 321.8 & 316.9 \\
\hline & MHV-26C & 0.4 & 0.1 & 13800 & 1.3 & 0.0 & Feb-93 & 48655.3 & 102404.2 & 359.8 & 331.8 & 326.9 \\
\hline & MHV-27A & 19.8 & 1.1 & 12900 & 19.5 & 10.6 & Feb-93 & 48654.5 & 102374.3 & 359.9 & 311.9 & 307.0 \\
\hline & MHV-27B & 165.0 & 29.0 & 12000 & 29.7 & 13.8 & Feb-93 & 48654.5 & 102374.3 & 359.9 & 321.9 & 317.0 \\
\hline & MHV-27C & 30.9 & 0.8 & 20400 & 0.6 & 0.0 & Feb-93 & 48654.5 & 102374.3 & 359.9 & 331.9 & 327.0 \\
\hline & MHV-28A & 0.7 & 0.2 & 3430 & 52.7 & 32.0 & Feb-93 & 48647.6 & 102366.3 & 359.9 & 311.9 & 307.0 \\
\hline & MHV-28B & 24.9 & 1.7 & 11600 & 48.1 & 13.2 & Feb-93 & 48647.6 & 102366.3 & 359.9 & 321.9 & 317.0 \\
\hline & MHV-28C & 114.0 & 0.3 & 30600 & 136.8 & 13.9 & Feb-93 & 48647.6 & 102366.3 & 359.9 & 331.9 & 327.0 \\
\hline & MHV-29A & 28.6 & 1.3 & 12200 & 51.4 & 25.9 & Feb-93 & 48661.8 & 102362.2 & 359.6 & 311.6 & 306.7 \\
\hline & MHV-29B & 68.2 & 12.4 & 9610 & 16.8 & 10.0 & Feb-93 & 48661.8 & 102362.2 & 359.6 & 321.6 & 316.7 \\
\hline & MHV-29C & 107.0 & 0.6 & 33600 & 224.4 & 18.8 & Feb-93 & 48661.8 & 102362.2 & 359.6 & 331.6 & 326.7 \\
\hline & CPTRAM15 ${ }^{\mathrm{C}}$ & 0.0 & 0.6 & 11800 & 53.7 & 40.4 & Mar-94 & 48600 & 102432.1 & 360.6 & Unknown & 270.6 \\
\hline & TUP- $1^{\mathrm{C}}$ & 36.9 & 5.6 & 15700 & & & & 48686.4 & 102407.0 & 360.0 & Unknown & 294.0 \\
\hline & TUP- $^{c}$ & 7.2 & 1.9 & 13200 & & & & 48686.4 & 102402.5 & 360.0 & Unknown & 269.0 \\
\hline & TUP-3 $^{\mathrm{C}}$ & 30.2 & 4.8 & 13500 & & & & 48686.4 & 102399.8 & 360.0 & Unknown & 296.2 \\
\hline & TUP- $4^{\text {C }}$ & 3.5 & 0.9 & 14600 & & & & 48686.4 & 102397.3 & 360.0 & Unknown & 264.0 \\
\hline
\end{tabular}

a - low $\mathrm{CO}_{2}$ usually indicates poor sample recovery

$\mathrm{b}$ - wells have multiple screen zones

$\mathrm{c}$ - bottom of wells were measured with probable screen lengths of 5 or $10 \mathrm{ft}$ 


\section{APPENDIX B - Current Sediment Concentrations}

\begin{tabular}{|c|c|c|c|c|c|c|}
\hline Boring & Depth, ft & \begin{tabular}{|l|} 
Elevation \\
$\mathrm{ft} \mathrm{msl}$
\end{tabular} & $\begin{array}{l}\text { PCE } \\
\mathrm{ug} / \mathrm{kg}\end{array}$ & $\begin{array}{c}\text { TCE } \\
\mathrm{ug} / \mathrm{kg}\end{array}$ & $\begin{array}{l}\text { Sediment } \\
\text { Description }\end{array}$ & Comments \\
\hline \multirow[t]{9}{*}{ MVB-101 } & 38 & 328.4 & 7.4 & 0.5 & sand & \\
\hline & 39 & 327.4 & 5.7 & 0.5 & sand & \\
\hline & 40 & 326.4 & 2.8 & 0.3 & sand & \\
\hline & 41 & 325.4 & 91.6 & 4.2 & sand & \\
\hline & 90 & 276.4 & 3.4 & 0.3 & sand/clay & \\
\hline & 91 & 275.4 & 1.5 & & sand & \\
\hline & 99 & 267.4 & 2.2 & 0.6 & sand & \\
\hline & 100 & 266.4 & 0.4 & & sand & \\
\hline & 104 & 262.4 & 5.6 & 2.8 & sand & CPT Refusal @ 104' \\
\hline \multirow[t]{17}{*}{ MVB-104 } & 24 & 339.7 & 1.3 & 0.5 & sand/clay & \\
\hline & 25 & 338.7 & 1.0 & 0.5 & sand/clay & \\
\hline & 35 & 328.7 & 3.4 & 0.2 & sand/clay & \\
\hline & 36 & 327.7 & 0.8 & 0.4 & sand/clay & \\
\hline & 37 & 326.7 & 0.4 & 0.3 & sand/clay & \\
\hline & 38 & 325.7 & 0.9 & 0.2 & sand/clay & \\
\hline & 39 & 324.7 & 0.3 & 0.2 & sand/clay & \\
\hline & 40 & 323.7 & 0.4 & 0.2 & sand/clay & \\
\hline & 85 & 278.7 & 0.6 & 0.5 & sand/clay & \\
\hline & 86 & 277.7 & 0.5 & 0.5 & sand & \\
\hline & 87 & 276.7 & 0.4 & 0.3 & sand & \\
\hline & 88 & 275.7 & 0.4 & 0.3 & sand & \\
\hline & 89 & 274.7 & 1.2 & 0.4 & sand & \\
\hline & 99 & 264.7 & 0.3 & 0.2 & sand & \\
\hline & 100 & 263.7 & 0.3 & 0.2 & sand/clay & \\
\hline & 101 & 262.7 & 0.3 & 0.2 & sand & \\
\hline & 102 & 261.7 & 8.1 & 9.5 & clay & CPT Refusal @ 103' \\
\hline \multirow[t]{16}{*}{ MVB-105 } & 24 & 339.8 & 2.5 & 1.2 & clay & \\
\hline & 25 & 338.8 & 0.6 & 0.2 & clay & \\
\hline & 35 & 328.8 & 54.5 & 1.1 & sand/clay & \\
\hline & 36 & 327.8 & 778.9 & 16.2 & clay & \\
\hline & 37 & 326.8 & 132.4 & 2.1 & sand/clay & \\
\hline & 38 & 325.8 & 784.3 & 16.8 & clay & \\
\hline & 39 & 324.8 & 9.7 & 0.3 & sand/clay & \\
\hline & 40 & 323.8 & 15.1 & 6.0 & sand & \\
\hline & 85 & 278.8 & 2.1 & 0.3 & sand/clay & \\
\hline & 86 & 277.8 & 1.9 & 0.4 & sand/clay & \\
\hline & 87 & 276.8 & 2.7 & 0.3 & sand/clay & \\
\hline & 88 & 275.8 & 2.7 & 2.0 & sand/clay & \\
\hline & 89 & 274.8 & 3.3 & 0.9 & sand/clay & \\
\hline & 99 & 264.8 & 2.0 & 0.4 & sand/clay & \\
\hline & 100 & 263.8 & 3.8 & 2.2 & sand/clay & \\
\hline & 101 & 262.8 & 3.3 & 2.6 & sand/clay & CPT Refusal @ 103' \\
\hline
\end{tabular}




\begin{tabular}{|c|c|c|c|c|c|c|}
\hline Boring & Depth, ft & $\begin{array}{l}\text { Elevation, } \\
\mathrm{ft} \mathrm{msl}\end{array}$ & $\begin{array}{l}\text { PCE, } \\
\mathrm{ug} / \mathrm{kg}\end{array}$ & $\begin{array}{l}\text { TCE, } \\
\mathrm{ug} / \mathrm{kg}\end{array}$ & $\begin{array}{l}\text { Sediment } \\
\text { Description }\end{array}$ & Comments \\
\hline \multirow[t]{16}{*}{ MVB-106 } & 24 & 337.7 & 0.8 & & sand/clay & \\
\hline & 25 & 336.7 & 0.5 & & sand/clay & \\
\hline & 35 & 326.7 & 9.1 & 0.2 & sand/clay & \\
\hline & 36 & 325.7 & 36.2 & 0.2 & sand/clay & \\
\hline & 37 & 324.7 & 19.2 & 0.2 & sand/clay & \\
\hline & 38 & 323.7 & 36.6 & 0.2 & sand/clay & \\
\hline & 39 & 322.7 & 1.4 & & sand/clay & \\
\hline & 40 & 321.7 & 3.7 & & sand/clay & \\
\hline & 85 & 276.7 & 0.3 & & sand/clay & \\
\hline & 86 & 275.7 & 0.6 & & sand/clay & \\
\hline & 87 & 274.7 & 0.3 & & sand/clay & \\
\hline & 88 & 273.7 & 0.7 & & sand/clay & \\
\hline & 89 & 272.7 & 1.2 & 0.3 & clay & \\
\hline & 99 & 262.7 & 6.1 & 1.3 & sand/clay & \\
\hline & 100 & 261.7 & 21.9 & 3.0 & sand/clay & \\
\hline & 101 & 260.7 & 14.8 & 2.3 & sand/clay & CPT Refusal @ 102' \\
\hline \multirow[t]{17}{*}{ MVB-107 } & 24 & 338.2 & 0.7 & & sand/clay & \\
\hline & 25 & 337.2 & 1.9 & & sand/clay & \\
\hline & 35 & 327.2 & 984.9 & 0.2 & clay & \\
\hline & 36 & 326.2 & 260.0 & 4.9 & clay & \\
\hline & 37 & 325.2 & 437.8 & 1.8 & clay & \\
\hline & 38 & 324.2 & 360.0 & 6.5 & clay & \\
\hline & 39 & 323.2 & 409.8 & & clay & \\
\hline & 40 & 322.2 & $1,214.1$ & 7.2 & clay & \\
\hline & 85 & 277.2 & 1.9 & 0.3 & sand/clay & \\
\hline & 86 & 276.2 & 1.2 & 0.6 & sand/clay & \\
\hline & 87 & 275.2 & 2.1 & 7.9 & clay & \\
\hline & 88 & 274.2 & 10.1 & 44.4 & clay & \\
\hline & 89 & 273.2 & 4.2 & 28.7 & sand & \\
\hline & 90 & 272.2 & 0.6 & 2.0 & sand & \\
\hline & 99 & 263.2 & 4.8 & 60.0 & sand/clay & \\
\hline & 100 & 262.2 & 11.0 & 23.7 & sand/clay & \\
\hline & 101 & 261.2 & 9.8 & 120.3 & sand/clay & CPT Refusal @ 102' \\
\hline
\end{tabular}




\begin{tabular}{|c|c|c|c|c|c|c|}
\hline Boring & Depth, ft & $\begin{array}{l}\text { Elevation, } \\
\mathrm{ft} \mathrm{msl}\end{array}$ & $\begin{array}{l}\mathrm{PCE}, \\
\mathrm{ug} / \mathrm{kg}\end{array}$ & $\begin{array}{l}\text { TCE, } \\
\mathrm{ug} / \mathrm{kg}\end{array}$ & $\begin{array}{l}\text { Sediment } \\
\text { Description }\end{array}$ & Comments \\
\hline \multirow[t]{16}{*}{ MVB-108 } & 24 & 339.6 & 458.0 & $1,110.3$ & sand/clay & \\
\hline & 25 & 338.6 & $1,595.9$ & $3,695.7$ & sand/clay & \\
\hline & 35 & 328.6 & 98.3 & 123.1 & clay & \\
\hline & 36 & 327.6 & 77.6 & 131.4 & sand/clay & \\
\hline & 37 & 326.6 & 71.7 & 96.3 & sand/clay & \\
\hline & 38 & 325.6 & 47.4 & 54.3 & clay & \\
\hline & 39 & 324.6 & 104.1 & 126.9 & clay & \\
\hline & 40 & 323.6 & 14.9 & 19.9 & clay & \\
\hline & 85 & 278.6 & 1.0 & 1.7 & sand/clay & \\
\hline & 86 & 277.6 & 1.1 & 1.1 & sand/clay & \\
\hline & 87 & 276.6 & 0.9 & 1.9 & sand/clay & \\
\hline & 88 & 275.6 & 0.7 & 1.2 & sand/clay & \\
\hline & 89 & 274.6 & 0.6 & 1.0 & sand/clay & \\
\hline & 99 & 264.6 & 67.2 & 662.3 & clay & \\
\hline & 100 & 263.6 & 44.7 & 370.5 & sand/clay & \\
\hline & 101 & 262.6 & 6.2 & 69.5 & sand/clay & CPT Refusal @ 102' \\
\hline \multirow[t]{14}{*}{ MVB-109 } & 38 & 327.1 & - & & sandy clay & \\
\hline & 39 & 326.1 & 0.1 & & sandy clay & \\
\hline & 40 & 325.1 & 0.1 & & sandy clay & \\
\hline & 41 & 324.1 & - & & clay & \\
\hline & 85 & 280.1 & - & & sand & \\
\hline & 86 & 279.1 & - & & sand & \\
\hline & 87 & 278.1 & - & & sand & \\
\hline & 88 & 277.1 & - & & sand & \\
\hline & 89 & 276.1 & - & & sandy clay & \\
\hline & 90 & 275.1 & 0.6 & 1.2 & clay & \\
\hline & 91 & 274.1 & 7.7 & 43.4 & clay & \\
\hline & 92 & 273.1 & 0.8 & 6.4 & sand/clay & \\
\hline & 93 & 272.1 & 3.6 & 33.5 & clay & \\
\hline & 94 & 271.1 & 3.1 & 29.9 & clay & CPT Refusal @ 121' \\
\hline \multirow[t]{14}{*}{ MVB-110 } & 35 & 330.5 & $1,126.8$ & $1,644.0$ & sand/clay & \\
\hline & 36 & 329.5 & 91.0 & 273.8 & sand & \\
\hline & 37 & 328.5 & 18.3 & 56.4 & sand & \\
\hline & 38 & 327.5 & 19.3 & 67.8 & sand & \\
\hline & 39 & 326.5 & 24.9 & 89.7 & sand & \\
\hline & 40 & 325.5 & 51.2 & 272.4 & sand & \\
\hline & 41 & 324.5 & 188.5 & 607.5 & sand/clay & \\
\hline & 62 & 303.5 & - & & sand/clay & \\
\hline & 63 & 302.5 & 0.8 & 2.6 & sand/clay & \\
\hline & 64 & 301.5 & 3.2 & 12.2 & sand/clay & \\
\hline & 65 & 300.5 & 0.5 & 2.9 & sand/clay & \\
\hline & 66 & 299.5 & 33.6 & 12.7 & sand/clay & \\
\hline & 95 & & & & no sample & perched water \\
\hline & 100 & & & & no sample & perched water \\
\hline
\end{tabular}




\begin{tabular}{|c|c|c|c|c|c|c|}
\hline Boring & Depth, ft & $\begin{array}{l}\text { Elevation, } \\
\mathrm{ft} \mathrm{msl}\end{array}$ & $\begin{array}{l}\text { PCE, } \\
\mathrm{ug} / \mathrm{kg}\end{array}$ & $\begin{array}{l}\text { TCE, } \\
\mathrm{ug} / \mathrm{kg} \\
\end{array}$ & \begin{tabular}{|l|} 
Sediment \\
Description
\end{tabular} & Comments \\
\hline \multirow[t]{8}{*}{ MVB-113 } & $\begin{array}{r}34 \\
\end{array}$ & 335.2 & 0.8 & 1.5 & clay & \\
\hline & 35 & 334.2 & 0.3 & 0.5 & sand/clay & \\
\hline & 64 & 305.2 & 0.4 & 0.7 & sand/clay & \\
\hline & 65 & 304.2 & 0.6 & 0.7 & sand/clay & \\
\hline & 99 & 270.2 & 78.6 & 610.0 & sand/clay & \\
\hline & 100 & 269.2 & 22.7 & 159.3 & sand & \\
\hline & 101 & 268.2 & 66.0 & 439.4 & sand/clay & \\
\hline & 102 & 267.2 & 19.9 & 118.9 & sand/clay & CPT Refusal @ 102' \\
\hline \multirow[t]{21}{*}{ MVB-114 } & 35 & 330.1 & 5.0 & 1.0 & sand/clay & \\
\hline & 36 & 329.1 & 2.3 & 0.7 & sand/clay & \\
\hline & 37 & 328.1 & 2.5 & 0.7 & sand/clay & \\
\hline & 38 & 327.1 & 1.5 & 3.3 & clay & \\
\hline & 39 & 326.1 & 0.8 & 1.8 & na & \\
\hline & 40 & 325.1 & 1.6 & 2.5 & clay & \\
\hline & 41 & 324.1 & 2.6 & 4.9 & na & \\
\hline & 65 & 300.1 & 0.2 & 0.6 & sand & \\
\hline & 66 & 299.1 & 0.4 & 0.8 & sand & \\
\hline & 67 & 298.1 & 0.3 & 0.5 & sand & \\
\hline & 68 & 297.1 & 0.1 & 0.2 & sand & \\
\hline & 69 & 296.1 & 0.2 & 0.5 & sand & \\
\hline & 70 & 295.1 & 1.3 & 1.8 & sand & \\
\hline & 71 & 294.1 & 0.2 & 0.4 & sand & \\
\hline & 72 & 293.1 & 0.2 & 0.5 & sand & \\
\hline & 73 & 292.1 & 0.4 & 4.3 & sand & \\
\hline & 74 & 291.1 & 0.4 & 5.3 & sand & \\
\hline & 95 & 270.1 & 0.6 & 5.7 & sand & \\
\hline & 96 & 269.1 & 44.4 & 456.5 & sand/clay & \\
\hline & 100 & 265.1 & 255.7 & $5,267.3$ & clay & \\
\hline & 101 & 264.1 & 298.8 & $6,562.7$ & clay & CPT Refusal @ 111' \\
\hline \multirow[t]{7}{*}{ MVB-117 } & 35 & 332.1 & 0.5 & 0.2 & sand/clay & \\
\hline & 64 & 303.1 & 0.5 & 0.2 & sand/clay & \\
\hline & 65 & 302.1 & 1.1 & 0.4 & sand/clay & \\
\hline & 99 & 268.1 & 0.7 & 1.0 & sand & \\
\hline & 100 & 267.1 & 0.3 & 0.4 & sand & \\
\hline & 101 & 266.1 & 0.4 & 0.4 & sand & \\
\hline & 102 & 265.1 & 106.4 & 377.1 & clay & CPT Refusal @ 103' \\
\hline
\end{tabular}




\begin{tabular}{|c|c|c|c|c|c|c|}
\hline Boring & Depth, $\mathrm{ft}$ & $\begin{array}{l}\text { Elevation, } \\
\mathrm{ft} \mathrm{ms}\end{array}$ & $\begin{array}{l}\text { PCE, } \\
\mathrm{ug} / \mathrm{kg}\end{array}$ & $\begin{array}{l}\text { TCE, } \\
\mathrm{ug} / \mathrm{kg}\end{array}$ & $\begin{array}{l}\text { Sediment } \\
\text { Description }\end{array}$ & Comments \\
\hline \multirow[t]{17}{*}{ MVB-118 } & $\begin{array}{r}19 \\
\end{array}$ & 344.5 & 0.4 & 0.2 & sand/clay & \\
\hline & 20 & 343.5 & 0.5 & & clay & \\
\hline & 21 & 342.5 & 0.3 & & clay & \\
\hline & 22 & 341.5 & 0.3 & & clay & \\
\hline & 37 & 326.5 & 0.3 & 0.2 & clay & \\
\hline & 38 & 325.5 & 0.7 & 0.3 & sand/clay & \\
\hline & 61 & 302.5 & 0.5 & & sand & \\
\hline & 62 & 301.5 & 0.3 & & sand & \\
\hline & 63 & 300.5 & 0.2 & - & sand & \\
\hline & 64 & 299.5 & 0.3 & & sand & \\
\hline & 91 & 272.5 & 0.3 & 0.8 & sand/clay & \\
\hline & 92 & 271.5 & 0.2 & 0.2 & sand/clay & \\
\hline & 93 & 270.5 & 0.3 & 0.7 & sand/clay & \\
\hline & 94 & 269.5 & 0.3 & 0.8 & clay & \\
\hline & 95 & 268.5 & 0.3 & & sand/clay & \\
\hline & 96 & 267.5 & 0.2 & 0.6 & sand/clay & \\
\hline & 97 & 266.5 & 0.2 & & sand & CPT Refusal @ 98' \\
\hline \multirow[t]{12}{*}{ MVB-119 } & 36 & 326.7 & 0.2 & & na & \\
\hline & 37 & 325.7 & 0.4 & 0.2 & clay & \\
\hline & 38 & 324.7 & 0.2 & 0.1 & clay & \\
\hline & 43 & 319.7 & 0.2 & & sand & \\
\hline & 44 & 318.7 & 0.2 & & sand & \\
\hline & 47 & 315.7 & 0.2 & & sand & \\
\hline & 53 & 309.7 & 0.2 & & sand & \\
\hline & 94 & 268.7 & 0.1 & & sand & \\
\hline & 95 & 267.7 & 0.2 & 0.5 & sand & \\
\hline & 96 & 266.7 & 0.5 & 2.0 & sand & \\
\hline & 97 & 265.7 & 0.2 & 0.7 & sand & \\
\hline & 98 & 264.7 & 55.3 & 84.5 & clay & CPT Refusal @ 102' \\
\hline \multirow[t]{15}{*}{ MVB-120 } & 35 & 326.8 & 3.7 & 2.1 & clay & \\
\hline & 36 & 325.8 & 2.5 & 1.5 & clay & \\
\hline & 37 & 324.8 & 1.2 & 1.1 & clay & \\
\hline & 42 & 319.8 & 0.7 & 0.9 & clay & \\
\hline & 43 & 318.8 & 0.5 & 0.6 & clay & \\
\hline & 44 & 317.8 & 0.6 & 0.7 & clay & \\
\hline & 45 & 316.8 & 0.6 & 0.4 & sand & \\
\hline & 51 & 310.8 & 0.5 & 0.3 & sand & \\
\hline & 52 & 309.8 & 1.0 & 0.4 & sand & \\
\hline & 92 & 269.8 & 32.3 & 9.6 & sand/clay & \\
\hline & 93 & 268.8 & 1.7 & 1.2 & sand/clay & \\
\hline & 94 & 267.8 & 7.6 & 3.1 & sand/clay & \\
\hline & 95 & 266.8 & 43.1 & 14.1 & sand/clay & \\
\hline & 96 & 265.8 & 8.1 & 4.2 & sand/clay & \\
\hline & 97 & 264.8 & 1.4 & 0.8 & sand/clay & CPT Refusal @ 98' \\
\hline
\end{tabular}


WSRC-TR-2001-00077

February 2001

\begin{tabular}{|c|c|c|c|c|c|c|}
\hline Boring & Depth, ft & $\begin{array}{l}\text { Elevation, } \\
\mathrm{ft} \mathrm{ms}\end{array}$ & $\begin{array}{l}\mathrm{PCE}, \\
\mathrm{ug} / \mathrm{kg}\end{array}$ & $\begin{array}{l}\text { TCE, } \\
\mathrm{ug} / \mathrm{kg}\end{array}$ & $\begin{array}{l}\text { Sediment } \\
\text { Description }\end{array}$ & Comments \\
\hline \multirow[t]{10}{*}{ MVB-121 } & $\begin{array}{r}31 \\
\end{array}$ & 325.7 & 22.3 & 2.3 & sand/clay & \\
\hline & 32 & 324.7 & 1.6 & 0.4 & sand/clay & \\
\hline & 33 & 323.7 & 2.1 & 0.5 & sand/clay & \\
\hline & 93 & 263.7 & 1.4 & 1.4 & sand & \\
\hline & 94 & 262.7 & 0.9 & 1.1 & sand & \\
\hline & 95 & 261.7 & 1.5 & 3.7 & sand/clay & \\
\hline & 96 & 260.7 & 0.7 & 0.9 & sand & \\
\hline & 97 & 259.7 & 0.5 & 0.3 & sand & \\
\hline & 98 & 258.7 & 0.4 & 0.2 & sand & \\
\hline & 99 & 257.7 & 0.5 & 0.2 & sand & CPT Refusal @ 100' \\
\hline \multirow[t]{8}{*}{ MVB-122 } & 36 & 324.3 & 55.8 & 13.8 & clay & \\
\hline & 37 & 323.3 & 33.7 & 13.2 & clay & \\
\hline & 38 & 322.3 & 81.4 & 22.7 & clay & \\
\hline & 39 & 321.3 & 100.9 & 34.0 & clay & \\
\hline & 40 & 320.3 & 1.0 & 0.2 & sand & \\
\hline & 59 & 301.3 & 0.6 & 0.2 & clay & \\
\hline & 60 & 300.3 & 0.3 & & clay & \\
\hline & 61 & 299.3 & 0.2 & & clay & CPT Refusal @ 82' \\
\hline \multirow[t]{15}{*}{ MVB-124 } & 33 & 326.7 & 7.3 & 0.9 & sand & \\
\hline & 34 & 325.7 & 3.3 & 0.4 & sand & \\
\hline & 35 & 324.7 & 32.9 & 1.6 & sand & \\
\hline & 36 & 323.7 & 15.7 & 5.2 & sand & \\
\hline & 37 & 322.7 & 34.4 & 0.9 & clay & \\
\hline & 38 & 321.7 & 48.6 & 2.1 & clay & \\
\hline & 60 & 299.7 & 0.8 & 0.7 & sand/clay & \\
\hline & 61 & 298.7 & 1.4 & 1.5 & sand/clay & \\
\hline & 62 & 297.7 & 1.3 & 3.7 & sand/clay & \\
\hline & 63 & 296.7 & 1.5 & 2.5 & sand/clay & \\
\hline & 64 & 295.7 & 1.7 & 0.6 & sand & \\
\hline & 65 & 294.7 & 1.9 & 0.5 & sand & \\
\hline & 90 & 269.7 & 1.3 & 1.6 & clay & very wet \\
\hline & 91 & 268.7 & 196.0 & 151.4 & clay & very wet \\
\hline & 92 & 267.7 & 98.8 & 82.5 & clay & very wet, abandoned push \\
\hline
\end{tabular}




\begin{tabular}{|c|c|c|c|c|c|c|}
\hline Boring & Depth, ft & $\begin{array}{l}\text { Elevation, } \\
\mathrm{ft} \mathrm{msl}\end{array}$ & $\begin{array}{l}\text { PCE, } \\
\mathrm{ug} / \mathrm{kg}\end{array}$ & $\begin{array}{l}\text { TCE, } \\
\mathrm{ug} / \mathrm{kg}\end{array}$ & \begin{tabular}{|l|} 
Sediment \\
Description
\end{tabular} & Comments \\
\hline \multirow[t]{13}{*}{ MVB-125 } & 33 & \begin{tabular}{|r|}
324.0 \\
\end{tabular} & 82.7 & 35.7 & clay & \\
\hline & 35 & 322.0 & 628.0 & 337.4 & clay & \\
\hline & 36 & 321.0 & 99.3 & 46.5 & sand & \\
\hline & 37 & 320.0 & 2.2 & 1.0 & sand & \\
\hline & 59 & 298.0 & 12.8 & 4.4 & sand/clay & \\
\hline & 60 & 297.0 & 90.3 & 32.4 & clay & \\
\hline & 61 & 296.0 & 74.0 & 57.6 & clay & \\
\hline & 62 & 295.0 & 179.7 & 96.8 & sand/clay & \\
\hline & 63 & 294.0 & 10.9 & 5.7 & sand/clay & \\
\hline & 85 & 272.0 & $3,816.7$ & $1,322.4$ & na & \\
\hline & 86 & 271.0 & $1,690.7$ & 908.2 & clay & \\
\hline & 87 & 270.0 & 899.4 & 593.9 & sand/clay & \\
\hline & 88 & 269.0 & 53.8 & 70.3 & sand & CPT Refusal @ 103' \\
\hline \multirow[t]{24}{*}{ MVB-126 } & 35 & 325.4 & 0.5 & 0.3 & clay & \\
\hline & 36 & 324.4 & 1.8 & 1.0 & clay & \\
\hline & 37 & 323.4 & 0.9 & 0.1 & clay & \\
\hline & 38 & 322.4 & 1.1 & 0.7 & clay & \\
\hline & 39 & 321.4 & 2.8 & 1.7 & clay & \\
\hline & 40 & 320.4 & 2.3 & 2.5 & clay & \\
\hline & 41 & 319.4 & 5.9 & 4.0 & clay & \\
\hline & 42 & 318.4 & 2.8 & 2.6 & clay & \\
\hline & 43 & 317.4 & 1.5 & 1.2 & clay & \\
\hline & 44 & 316.4 & 0.8 & 0.9 & clay & \\
\hline & 62 & 298.4 & 5.4 & 3.7 & na & \\
\hline & 63 & 297.4 & 6.9 & 5.0 & clay & \\
\hline & 64 & 296.4 & 0.2 & 0.3 & sand/clay & \\
\hline & 65 & 295.4 & 1.4 & 1.5 & sand/clay & \\
\hline & 66 & 294.4 & 1.6 & 1.7 & sand/clay & \\
\hline & 88 & 272.4 & 0.1 & 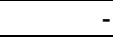 & sand & \\
\hline & 89 & 271.4 & 0.1 & & sand & \\
\hline & 90 & 270.4 & 0.4 & 0.5 & sand/clay & \\
\hline & 91 & 269.4 & 0.6 & 0.8 & sand/clay & \\
\hline & 105 & 255.4 & 71.9 & 67.1 & sand & \\
\hline & 106 & 254.4 & 0.2 & 0.2 & sand & \\
\hline & 107 & 253.4 & 0.1 & & sand & \\
\hline & 108 & 252.4 & 0.1 & & sand & \\
\hline & 109 & 251.4 & 4.4 & 10.6 & sand/clay & CPT Refusal @ 110' \\
\hline
\end{tabular}




\begin{tabular}{|c|c|c|c|c|c|c|}
\hline Boring & Depth, ft & \begin{tabular}{|l|} 
Elevation, \\
$\mathrm{ft}$ msl
\end{tabular} & $\begin{array}{l}\mathrm{PCE}, \\
\mathrm{ug} / \mathrm{kg}\end{array}$ & $\begin{array}{l}\text { TCE, } \\
\mathrm{ug} / \mathrm{kg}\end{array}$ & \begin{tabular}{|l|} 
Sediment \\
Description
\end{tabular} & Comments \\
\hline \multirow[t]{15}{*}{ MVB-129 } & 28 & 332.4 & 918.6 & & sand/clay & \\
\hline & 29 & 331.4 & $4,252.1$ & & sand/clay & \\
\hline & 30 & 330.4 & $6,040.6$ & & na & \\
\hline & 40 & 320.4 & 157.7 & & sand/clay & \\
\hline & 41 & 319.4 & 62.3 & & na & \\
\hline & 42 & 318.4 & 150.4 & & na & \\
\hline & 43 & 317.4 & 301.9 & & sand/clay & \\
\hline & 60 & 300.4 & 338.2 & & sand/clay & \\
\hline & 88 & 272.4 & 5.7 & 0.2 & sand/clay & \\
\hline & 89 & 271.4 & 13.7 & 3.4 & sand/clay & \\
\hline & 90 & 270.4 & 11.1 & 1.7 & sand/clay & \\
\hline & 91 & 269.4 & 713.4 & 358.0 & clay & \\
\hline & 105 & 255.4 & 29.6 & 1.0 & sand & \\
\hline & 108 & 252.4 & 9.9 & 1.1 & sand & \\
\hline & 109 & 251.4 & 31.4 & 7.6 & clay & CPT Refusal @ 110' \\
\hline \multirow[t]{31}{*}{ MRS-37 } & 15 & 332.8 & 0.5 & 0.5 & & Roto Sonic Core \\
\hline & 16 & 331.8 & 0.5 & 0.5 & & \\
\hline & 17 & 330.8 & 0.5 & 0.5 & & \\
\hline & 23 & 324.8 & 1.4 & 300.9 & & \\
\hline & 24 & 323.8 & 0.5 & 19.0 & & \\
\hline & 31 & 316.8 & 0.5 & 3.0 & & \\
\hline & 35 & 312.8 & 3.1 & 478.2 & & \\
\hline & 43 & 304.8 & 5.1 & 28.4 & & \\
\hline & 44 & 303.8 & 33.3 & 148.0 & & \\
\hline & 47 & 300.8 & 0.6 & 3.1 & & \\
\hline & 50 & 297.8 & 1.9 & 30.8 & & \\
\hline & 62 & 285.8 & 35.3 & 120.6 & & \\
\hline & 64 & 283.8 & 3.6 & 20.5 & & \\
\hline & 65 & 282.8 & 11.0 & \begin{tabular}{|l|}
62.4 \\
\end{tabular} & & \\
\hline & 72 & 275.8 & 0.5 & 1.9 & & \\
\hline & 77 & 270.8 & 0.5 & 0.9 & & \\
\hline & 82 & 265.8 & 0.5 & 0.5 & & \\
\hline & 90 & 257.8 & $2,450.7$ & $2,055.1$ & & \\
\hline & 93 & 254.8 & 644.2 & 304.4 & & \\
\hline & 96 & 251.8 & 7.0 & 3.0 & & \\
\hline & 98 & 249.8 & $5,045.8$ & $3,780.1$ & & \\
\hline & 102 & 245.8 & 12.3 & 13.5 & & \\
\hline & 104 & 243.8 & 6.0 & 3.4 & & \\
\hline & 111 & 236.8 & 17.1 & 13.5 & & \\
\hline & 116 & 231.8 & 1.0 & 1.3 & & \\
\hline & 121 & 226.8 & 0.5 & 0.5 & & \\
\hline & 124 & 223.8 & 0.6 & 0.5 & & \\
\hline & 126 & 221.8 & 0.7 & 0.5 & & \\
\hline & 128 & 219.8 & 0.5 & 0.5 & & \\
\hline & 132 & 215.8 & 1.2 & 2.1 & & \\
\hline & 140 & 207.8 & 69.1 & 179.0 & & \\
\hline
\end{tabular}


Appendix C - Current CPT Soil Gas Concentrations

\begin{tabular}{|c|c|c|c|c|c|c|c|}
\hline Boring & SRS North & SRS East & $\begin{array}{c}\text { Depth } \\
\mathrm{ft}\end{array}$ & $\begin{array}{c}\text { Elevation } \\
\mathrm{ft} \text { msl }\end{array}$ & $\begin{array}{l}\text { PCE } \\
\text { ppmv }\end{array}$ & $\begin{array}{l}\text { TCE } \\
\text { ppmv }\end{array}$ & $\begin{array}{c}\mathrm{CO}_{2} \\
\mathrm{ppmv}\end{array}$ \\
\hline MVB-101 & 103639.9 & 49005.0 & 20.00 & 346.38 & 2.8 & 1.9 & 1,280 \\
\hline MVB-101 & 103639.9 & 49005.0 & 30.00 & 336.38 & 155.0 & 7.0 & 16,300 \\
\hline MVB-101 & 103639.9 & 49005.0 & 37.00 & 329.38 & 167.0 & 9.6 & 13,900 \\
\hline MVB-101 & 103639.9 & 49005.0 & 50.00 & 316.38 & 127.0 & 6.7 & 13,400 \\
\hline MVB-101 & 103639.9 & 49005.0 & 70.00 & 296.38 & 3.4 & 1.5 & 10,500 \\
\hline MVB-101 & 103639.9 & 49005.0 & 80.00 & 286.38 & 3.4 & 1.6 & 10,400 \\
\hline MVB-101 & 103639.9 & 49005.0 & 90.00 & 276.38 & 55.3 & 3.9 & 10,000 \\
\hline MVB-101 & 103639.9 & 49005.0 & 99.00 & 267.38 & 28.2 & 2.9 & 11,600 \\
\hline MVB-101 & 103639.9 & 49005.0 & 104.00 & 262.38 & 24.7 & 2.9 & 12,200 \\
\hline MVB-104 & 103440.8 & 49090.0 & 20.00 & 343.68 & 1.5 & 2.8 & 917 \\
\hline MVB-104 & 103440.8 & 49090.0 & 30.00 & 333.68 & 1.8 & 2.7 & 775 \\
\hline MVB-104 & 103440.8 & 49090.0 & 50.00 & 313.68 & 1.3 & 3.1 & 6,710 \\
\hline MVB-104 & 103440.8 & 49090.0 & 60.00 & 303.68 & 1.3 & 3.1 & 7,580 \\
\hline MVB-104 & 103440.8 & 49090.0 & 70.00 & 293.68 & 1.5 & 3.1 & 7,260 \\
\hline MVB-104 & 103440.8 & 49090.0 & 80.00 & 283.68 & 1.7 & 3.4 & 7,690 \\
\hline MVB-105 & 103425.1 & 49005.0 & 20.00 & 343.79 & 2.6 & 2.8 & 1,500 \\
\hline MVB-105 & 103425.1 & 49005.0 & 30.00 & 333.79 & 258.0 & 37.0 & 15,600 \\
\hline MVB-105 & 103425.1 & 49005.0 & 50.00 & 313.79 & 222.0 & 4.7 & 11,000 \\
\hline MVB-105 & 103425.1 & 49005.0 & 60.00 & 303.79 & 11.4 & 3.4 & 8,130 \\
\hline MVB-105 & 103425.1 & 49005.0 & 70.00 & 293.79 & 6.2 & 3.3 & 8,060 \\
\hline MVB-105 & 103425.1 & 49005.0 & 80.00 & 283.79 & 5.2 & 3.3 & 8,330 \\
\hline MVB-105 & 103425.1 & 49005.0 & 90.00 & 273.79 & 29.3 & 6.4 & 12,700 \\
\hline MVB-105 & 103425.1 & 49005.0 & 102.00 & 261.79 & 9.5 & 10.5 & 1,500 \\
\hline MVB-106 & 103262.4 & 49139.3 & 20.00 & 341.71 & 2.1 & 2.7 & 898 \\
\hline MVB-106 & 103262.4 & 49139.3 & 30.00 & 331.71 & 1.6 & 2.6 & 971 \\
\hline MVB-106 & 103262.4 & 49139.3 & 50.00 & 311.71 & 3.5 & 3.0 & 6,530 \\
\hline MVB-106 & 103262.4 & 49139.3 & 60.00 & 301.71 & 1.9 & 2.9 & 6,710 \\
\hline MVB-106 & 103262.4 & 49139.3 & 70.00 & 291.71 & 1.5 & 2.7 & 7,010 \\
\hline MVB-106 & 103262.4 & 49139.3 & 80.00 & 281.71 & 1.9 & 3.3 & 6,490 \\
\hline MVB-106 & 103262.4 & 49139.3 & 90.00 & 271.71 & 2.7 & 3.0 & 5,630 \\
\hline MVB-106 & 103262.4 & 49139.3 & 102.00 & 259.71 & 2.3 & 2.7 & 6,160 \\
\hline MVB-107 & 103249.9 & 48980.0 & 20.00 & 342.23 & 1.5 & 2.4 & 2,510 \\
\hline MVB-107 & 103249.9 & 48980.0 & 30.00 & 332.23 & 1.5 & 2.4 & 2,240 \\
\hline MVB-107 & 103249.9 & 48980.0 & 50.00 & 312.23 & 90.7 & 6.6 & 10,800 \\
\hline MVB-107 & 103249.9 & 48980.0 & 60.00 & 302.23 & 5.5 & 3.3 & 7,930 \\
\hline MVB-107 & 103249.9 & 48980.0 & 70.00 & 292.23 & 2.7 & 2.8 & 7,610 \\
\hline MVB-107 & 103249.9 & 48980.0 & 80.00 & 282.23 & 3.5 & 3.0 & 7,540 \\
\hline MVB-108 & 103086.9 & 48930.9 & 20.00 & 343.57 & 1.6 & 2.5 & 1,930 \\
\hline MVB-108 & 103086.9 & 48930.9 & 30.00 & 333.57 & 478.0 & 519.0 & 13,200 \\
\hline MVB-108 & 103086.9 & 48930.9 & 50.00 & 313.57 & 23.1 & 26.1 & 9,090 \\
\hline MVB-108 & 103086.9 & 48930.9 & 60.00 & 303.57 & 18.3 & 21.1 & 8,430 \\
\hline MVB-108 & 103086.9 & 48930.9 & 70.00 & 293.57 & 2.7 & 5.1 & 8,780 \\
\hline MVB-108 & 103086.9 & 48930.9 & 80.00 & 283.57 & 4.3 & 10.7 & 9,010 \\
\hline MVB-108 & 103086.9 & 48930.9 & 90.00 & 273.57 & 3.8 & 13.4 & 7,090 \\
\hline MVB-108 & 103086.9 & 48930.9 & 102.00 & 261.57 & 39.2 & 114.0 & 10,100 \\
\hline
\end{tabular}


WSRC-TR-2001-00077

February 2001

\begin{tabular}{|c|c|c|c|c|c|c|c|}
\hline Boring & SRS North & SRS East & $\begin{array}{c}\text { Depth } \\
\mathrm{ft}\end{array}$ & $\begin{array}{c}\text { Elevation } \\
\mathrm{ft} \mathrm{msl}\end{array}$ & $\begin{array}{l}\text { PCE } \\
\text { ppmv }\end{array}$ & $\begin{array}{l}\text { TCE } \\
\text { ppmv }\end{array}$ & $\begin{array}{l}\mathrm{CO}_{2} \\
\mathrm{ppmv}\end{array}$ \\
\hline MVB-109 & 103070.0 & 49060.0 & 20.00 & 345.08 & 0.8 & 1.7 & 3,010 \\
\hline MVB-109 & 103070.0 & 49060.0 & 30.00 & 335.08 & 1.0 & 20.9 & 3,310 \\
\hline MVB-109 & 103070.0 & 49060.0 & 37.00 & 328.08 & 1.0 & 2.0 & 16,100 \\
\hline MVB-109 & 103070.0 & 49060.0 & 50.00 & 315.08 & 1.4 & 2.3 & 17,800 \\
\hline MVB-109 & 103070.0 & 49060.0 & 60.00 & 305.08 & 0.7 & 1.6 & 15,100 \\
\hline MVB-109 & 103070.0 & 49060.0 & 70.00 & 295.08 & 0.5 & 1.4 & 11,300 \\
\hline MVB-109 & 103070.0 & 49060.0 & 80.00 & 285.08 & 0.5 & 1.4 & 10,500 \\
\hline MVB-109 & 103070.0 & 49060.0 & 124.00 & 241.08 & 4.5 & 5.7 & 10,300 \\
\hline MVB-110 & 102952.2 & 48879.0 & 20.00 & 345.53 & 3.7 & 3.4 & 4,260 \\
\hline MVB-110 & 102952.2 & 48879.0 & 30.00 & 335.53 & 5.3 & 4.5 & 3,140 \\
\hline MVB-110 & 102952.2 & 48879.0 & 35.00 & 330.53 & 4.2 & 2.3 & 2,030 \\
\hline MVB-110 & 102952.2 & 48879.0 & 50.00 & 315.53 & 9.0 & 17.5 & 8,140 \\
\hline MVB-110 & 102952.2 & 48879.0 & 60.00 & 305.53 & 8.4 & 6.4 & 7,300 \\
\hline MVB-110 & 102952.2 & 48879.0 & 70.00 & 295.53 & 6.9 & 6.1 & 5,500 \\
\hline MVB-110 & 102952.2 & 48879.0 & 80.00 & 285.53 & 4.0 & 4.9 & 7,240 \\
\hline MVB-110 & 102952.2 & 48879.0 & 90.00 & 275.53 & 4.0 & 6.2 & 6,810 \\
\hline MVB-110 & 102952.2 & 48879.0 & 95.00 & 270.53 & 4.4 & 5.4 & 2,840 \\
\hline MVB-113 & 102854.1 & 48958.9 & 20.00 & 349.16 & 2.0 & 3.5 & 6,490 \\
\hline MVB-113 & 102854.1 & 48958.9 & 30.00 & 339.16 & 1.8 & 2.9 & 5,100 \\
\hline MVB-113 & 102854.1 & 48958.9 & 40.00 & 329.16 & 1.6 & 2.8 & 4,320 \\
\hline MVB-113 & 102854.1 & 48958.9 & 50.00 & 319.16 & 1.5 & 3.3 & 16,000 \\
\hline MVB-113 & 102854.1 & 48958.9 & 60.00 & 309.16 & 1.7 & 3.1 & 12,200 \\
\hline MVB-113 & 102854.1 & 48958.9 & 70.00 & 299.16 & 1.5 & 3.0 & 9,640 \\
\hline MVB-113 & 102854.1 & 48958.9 & 80.00 & 289.16 & 1.5 & 2.8 & 11,700 \\
\hline MVB-113 & 102854.1 & 48958.9 & 90.00 & 279.16 & 1.5 & 2.9 & 11,300 \\
\hline MVB-114 & 102802.7 & 48821.5 & 20.00 & 345.08 & 3.6 & 1.5 & 2,040 \\
\hline MVB-114 & 102802.7 & 48821.5 & 30.00 & 335.08 & 43.8 & 5.0 & 10,300 \\
\hline MVB-114 & 102802.7 & 48821.5 & 42.00 & 323.08 & 15.4 & 4.0 & 11,200 \\
\hline MVB-114 & 102802.7 & 48821.5 & 50.00 & 315.08 & 11.5 & 3.5 & 15,000 \\
\hline MVB-114 & 102802.7 & 48821.5 & 60.00 & 305.08 & 18.9 & 3.3 & 9,080 \\
\hline MVB-114 & 102802.7 & 48821.5 & 80.00 & 285.08 & 3.9 & 18.8 & 21,000 \\
\hline MVB-114 & 102802.7 & 48821.5 & 90.00 & 275.08 & 17.8 & 4.9 & 11,500 \\
\hline MVB-114 & 102802.7 & 48821.5 & 100.00 & 265.08 & 40.0 & 34.0 & 11,400 \\
\hline MVB-114 & 102802.7 & 48821.5 & 105.00 & 260.08 & 10.8 & 41.7 & 11,800 \\
\hline MVB-117 & 102778.2 & 48989.7 & 20.00 & 347.14 & 1.4 & 2.5 & 2,650 \\
\hline MVB-117 & 102778.2 & 48989.7 & 30.00 & 337.14 & 1.5 & 2.6 & 3,030 \\
\hline MVB-117 & 102778.2 & 48989.7 & 40.00 & 327.14 & 1.2 & 2.5 & 11,100 \\
\hline MVB-117 & 102778.2 & 48989.7 & 50.00 & 317.14 & 1.6 & 2.2 & 16,000 \\
\hline MVB-117 & 102778.2 & 48989.7 & 60.00 & 307.14 & 1.7 & 2.3 & 14,400 \\
\hline MVB-117 & 102778.2 & 48989.7 & 70.00 & 297.14 & 1.6 & 2.4 & 15,900 \\
\hline MVB-117 & 102778.2 & 48989.7 & 80.00 & 287.14 & 1.6 & 2.6 & 16,400 \\
\hline MVB-117 & 102778.2 & 48989.7 & 90.00 & 277.14 & 1.7 & 3.3 & 15,900 \\
\hline MVB-118 & 102720.0 & 48650.0 & 19.00 & 344.48 & 2.3 & 2.4 & 593 \\
\hline MVB-118 & 102720.0 & 48650.0 & 30.00 & 333.48 & 1.5 & 2.3 & 14,700 \\
\hline MVB-118 & 102720.0 & 48650.0 & 37.00 & 326.48 & 1.5 & 2.4 & 15,800 \\
\hline MVB-118 & 102720.0 & 48650.0 & 50.00 & 313.48 & 1.3 & 2.4 & 17,400 \\
\hline MVB-118 & 102720.0 & 48650.0 & 60.00 & 303.48 & 1.2 & 2.4 & 17,900 \\
\hline
\end{tabular}


WSRC-TR-2001-00077

February 2001

\begin{tabular}{|c|c|c|c|c|c|c|c|}
\hline Boring & SRS North & SRS East & $\begin{array}{c}\text { Depth } \\
\mathrm{ft}\end{array}$ & $\begin{array}{c}\text { Elevation } \\
\mathrm{ft} \mathrm{msl}\end{array}$ & $\begin{array}{l}\text { PCE } \\
\text { ppmv }\end{array}$ & $\begin{array}{l}\text { TCE } \\
\text { ppmv }\end{array}$ & $\begin{array}{l}\mathrm{CO}_{2} \\
\mathrm{ppmv}\end{array}$ \\
\hline MVB-118 & 102720.0 & 48650.0 & 70.00 & 293.48 & 1.0 & 2.3 & 18,200 \\
\hline MVB-118 & 102720.0 & 48650.0 & 80.00 & 283.48 & 1.1 & 2.4 & 18,300 \\
\hline MVB-118 & 102720.0 & 48650.0 & 90.00 & 273.48 & 1.1 & 2.5 & 18,000 \\
\hline MVB-119 & 102710.0 & 48750.0 & 20.00 & 342.72 & 1.4 & 2.4 & 3,460 \\
\hline MVB-119 & 102710.0 & 48750.0 & 30.00 & 332.72 & 1.2 & 2.4 & 18,500 \\
\hline MVB-119 & 102710.0 & 48750.0 & 40.00 & 322.72 & 3.5 & 5.2 & 16,000 \\
\hline MVB-119 & 102710.0 & 48750.0 & 50.00 & 312.72 & 1.2 & 2.6 & 16,500 \\
\hline MVB-119 & 102710.0 & 48750.0 & 60.00 & 302.72 & 1.9 & 3.3 & 12,400 \\
\hline MVB-119 & 102710.0 & 48750.0 & 70.00 & 292.72 & 0.9 & 2.4 & 18,300 \\
\hline MVB-119 & 102710.0 & 48750.0 & 80.00 & 282.72 & 0.9 & 2.4 & 17,900 \\
\hline MVB-119 & 102710.0 & 48750.0 & 90.00 & 272.72 & 1.1 & 2.7 & 14,200 \\
\hline MVB-119 & 102710.0 & 48750.0 & 101.00 & 261.72 & 1.3 & 5.3 & 20,200 \\
\hline MVB-120 & 102690.0 & 48875.0 & 20.00 & 341.77 & 1.7 & 2.7 & 1,260 \\
\hline MVB-120 & 102690.0 & 48875.0 & 30.00 & 331.77 & 1.4 & 2.8 & 15,800 \\
\hline MVB-120 & 102690.0 & 48875.0 & 40.00 & 321.77 & 1.5 & 2.8 & 8,660 \\
\hline MVB-120 & 102690.0 & 48875.0 & 50.00 & 311.77 & 3.1 & 3.0 & 14,900 \\
\hline MVB-120 & 102690.0 & 48875.0 & 60.00 & 301.77 & 4.0 & 3.2 & 13,300 \\
\hline MVB-120 & 102690.0 & 48875.0 & 70.00 & 291.77 & 4.7 & 3.2 & 13,700 \\
\hline MVB-120 & 102690.0 & 48875.0 & 80.00 & 281.77 & 4.0 & 3.2 & 12,700 \\
\hline MVB-120 & 102690.0 & 48875.0 & 90.00 & 271.77 & 2.8 & 3.0 & 10,700 \\
\hline MVB-121 & 102610.0 & 48860.0 & 20.00 & 336.66 & 1.9 & 2.3 & 2,000 \\
\hline MVB-121 & 102610.0 & 48860.0 & 30.00 & 326.66 & 2.6 & 2.6 & 13,600 \\
\hline MVB-121 & 102610.0 & 48860.0 & 40.00 & 316.66 & 75.6 & 12.3 & 24,000 \\
\hline MVB-121 & 102610.0 & 48860.0 & 50.00 & 306.66 & 19.6 & 5.8 & 12,900 \\
\hline MVB-121 & 102610.0 & 48860.0 & 60.00 & 296.66 & 51.4 & 16.5 & 10,600 \\
\hline MVB-121 & 102610.0 & 48860.0 & 70.00 & 286.66 & 69.6 & 21.2 & 11,400 \\
\hline MVB-121 & 102610.0 & 48860.0 & 80.00 & 276.66 & 16.6 & 9.5 & 12,400 \\
\hline MVB-121 & 102610.0 & 48860.0 & 90.00 & 266.66 & 16.2 & 8.6 & 12,500 \\
\hline MVB-122 & 102610.0 & 48720.0 & 20.00 & 340.26 & 7.4 & 3.9 & 12,500 \\
\hline MVB-122 & 102610.0 & 48720.0 & 30.00 & 330.26 & 48.5 & 9.2 & 29,700 \\
\hline MVB-122 & 102610.0 & 48720.0 & 41.00 & 319.26 & 13.6 & 5.5 & 17,000 \\
\hline MVB-122 & 102610.0 & 48720.0 & 50.00 & 310.26 & 6.6 & 3.3 & 13,700 \\
\hline MVB-122 & 102610.0 & 48720.0 & 62.00 & 298.26 & 1.7 & 2.5 & 12,900 \\
\hline MVB-122 & 102610.0 & 48720.0 & 70.00 & 290.26 & 3.5 & 5.0 & 12,900 \\
\hline MVB-122 & 102610.0 & 48720.0 & 80.00 & 280.26 & 2.8 & 3.2 & 13,100 \\
\hline MVB-124 & 102485.0 & 48670.0 & 20.00 & 339.73 & 133.0 & 14.3 & 13,100 \\
\hline MVB-124 & 102485.0 & 48670.0 & 30.00 & 329.73 & 129.0 & 18.4 & 28,200 \\
\hline MVB-124 & 102485.0 & 48670.0 & 39.00 & 320.73 & 162.0 & 28.2 & 22,100 \\
\hline MVB-124 & 102485.0 & 48670.0 & 50.00 & 309.73 & 47.4 & 11.6 & 20,900 \\
\hline MVB-124 & 102485.0 & 48670.0 & 60.00 & 299.73 & 25.2 & 6.4 & 19,400 \\
\hline MVB-124 & 102485.0 & 48670.0 & 70.00 & 289.73 & 5.3 & 2.9 & 13,200 \\
\hline MVB-124 & 102485.0 & 48670.0 & 80.00 & 279.73 & 4.2 & 2.7 & 13,000 \\
\hline MVB-125 & 102437.5 & 48723.3 & 20.00 & 336.99 & 2.0 & 2.4 & 2,130 \\
\hline MVB-125 & 102437.5 & 48723.3 & 30.00 & 326.99 & 4.0 & 2.7 & 5,740 \\
\hline MVB-125 & 102437.5 & 48723.3 & 40.00 & 316.99 & 2.6 & 2.7 & 2,370 \\
\hline MVB-125 & 102437.5 & 48723.3 & 50.00 & 306.99 & 20.5 & 4.6 & 12,300 \\
\hline MVB-125 & 102437.5 & 48723.3 & 70.00 & 286.99 & 2.5 & 2.7 & 15,000 \\
\hline
\end{tabular}


WSRC-TR-2001-00077

February 2001

\begin{tabular}{|l|r|r|r|r|r|r|r|}
\hline \multicolumn{1}{|c|}{ Boring } & SRS North & SRS East & $\begin{array}{c}\text { Depth } \\
\mathrm{ft}\end{array}$ & $\begin{array}{c}\text { Elevation } \\
\mathrm{ft} \mathrm{msl}\end{array}$ & $\begin{array}{c}\text { PCE } \\
\text { ppmv }\end{array}$ & $\begin{array}{c}\text { TCE } \\
\text { ppmv }\end{array}$ & $\begin{array}{c}\mathrm{CO}_{2} \\
\text { ppmv }\end{array}$ \\
\hline MVB-125 & 102437.5 & 48723.3 & 80.00 & 276.99 & 2.4 & 2.9 & 13,900 \\
\hline MVB-125 & 102437.5 & 48723.3 & 90.00 & 266.99 & 21.2 & 24.2 & 18,500 \\
\hline MVB-125 & 102437.5 & 48723.3 & 100.00 & 256.99 & 9.1 & 12.7 & 18,200 \\
\hline MVB-126 & 102407.5 & 48627.9 & 20.00 & 340.37 & 1.3 & 1.9 & 608 \\
\hline MVB-126 & 102407.5 & 48627.9 & 30.00 & 330.37 & 2.5 & 3.7 & 19,900 \\
\hline MVB-126 & 102407.5 & 48627.9 & 50.00 & 310.37 & 4.7 & 3.7 & 21,300 \\
\hline MVB-126 & 102407.5 & 48627.9 & 60.00 & 300.37 & 3.5 & 3.4 & 19,100 \\
\hline MVB-126 & 102407.5 & 48627.9 & 70.00 & 290.37 & 1.2 & 2.6 & 17,200 \\
\hline MVB-126 & 102407.5 & 48627.9 & 80.00 & 280.37 & 1.1 & 2.6 & 16,800 \\
\hline MVB-126 & 102407.5 & 48627.9 & 100.00 & 260.37 & 59.0 & 71.0 & 11,200 \\
\hline MVB-129 & 102249.9 & 48600.1 & 20.00 & 340.42 & 1.5 & 2.7 & 892 \\
\hline MVB-129 & 102249.9 & 48600.1 & 40.00 & 320.42 & $2,480.0$ & 23.9 & 11,300 \\
\hline MVB-129 & 102249.9 & 48600.1 & 50.00 & 310.42 & $1,630.0$ & 10.1 & 10,700 \\
\hline MVB-129 & 102249.9 & 48600.1 & 60.00 & 300.42 & $1,970.0$ & 15.3 & 9,160 \\
\hline MVB-129 & 102249.9 & 48600.1 & 70.00 & 290.42 & 187.0 & 2.7 & 14,100 \\
\hline MVB-129 & 102249.9 & 48600.1 & 80.00 & 280.42 & 348.0 & 2.7 & 13,900 \\
\hline MVB-129 & 102249.9 & 48600.1 & 100.00 & 260.42 & $1,920.0$ & 15.6 & 10,900 \\
\hline
\end{tabular}




\section{Appendix D - Initial Sediment Concentration}

Sample dates correspond to installation dates in Appendix E

\begin{tabular}{|c|c|c|c|c|c|c|}
\hline ID & $\begin{array}{c}\text { SRS } \\
\text { North } \\
\end{array}$ & $\begin{array}{l}\text { SRS } \\
\text { East } \\
\end{array}$ & $\begin{array}{c}\text { Sample } \\
\text { Depth } \\
\mathrm{ft}\end{array}$ & $\begin{array}{c}\text { Sample } \\
\text { Elevation } \\
\mathrm{ft} \mathrm{msl}\end{array}$ & $\begin{array}{c}\text { PCE } \\
(\mu \mathrm{g} / \mathrm{kg})\end{array}$ & $\begin{array}{c}\text { TCE } \\
(\mu \mathrm{g} / \mathrm{kg})\end{array}$ \\
\hline MHT-1C & 102706.8 & 48765.6 & 3 & 359.7 & - & - \\
\hline MHT-1C & 102706.8 & 48765.6 & 5 & 357.7 & - & - \\
\hline MHT-1C & 102706.8 & 48765.6 & 9 & 353.7 & - & - \\
\hline MHT-1C & 102706.8 & 48765.6 & 17 & 345.7 & 58.0 & 23.0 \\
\hline MHT-1C & 102706.8 & 48765.6 & 25 & 337.7 & 565.0 & 312.0 \\
\hline MHT-1C & 102706.8 & 48765.6 & 29 & 333.7 & 361.0 & 252.0 \\
\hline MHT-1C & 102706.8 & 48765.6 & 35 & 327.7 & $1,434.0$ & $1,082.0$ \\
\hline MHT-1C & 102706.8 & 48765.6 & 37 & 325.7 & $3,461.0$ & $1,496.0$ \\
\hline MHT-1C & 102706.8 & 48765.6 & 43 & 319.7 & 375.0 & 139.0 \\
\hline MHT-1C & 102706.8 & 48765.6 & 53 & 309.7 & 819.0 & $1,033.0$ \\
\hline MHT-1C & 102706.8 & 48765.6 & 61.5 & 301.2 & - & - \\
\hline MHT-1C & 102706.8 & 48765.6 & 73 & 289.7 & 216.0 & 109.0 \\
\hline MHT-1C & 102706.8 & 48765.6 & 83 & 279.7 & 159.0 & 13.0 \\
\hline MHT-1C & 102706.8 & 48765.6 & 89 & 273.7 & $2,333.0$ & $5,755.0$ \\
\hline MHT-1C & 102706.8 & 48765.6 & 93 & 269.7 & 197.0 & 557.0 \\
\hline MHT-1C & 102706.8 & 48765.6 & 98 & 264.7 & $3,391.0$ & $11,491.0$ \\
\hline MHT-1C & 102706.8 & 48765.6 & 105 & 257.7 & - & - \\
\hline MHT-1C & 102706.8 & 48765.6 & 107 & 255.7 & - & - \\
\hline MHT-1C & 102706.8 & 48765.6 & 113 & 249.7 & - & 12.0 \\
\hline MHT-1C & 102706.8 & 48765.6 & 118 & 244.7 & 654.0 & $1,924.0$ \\
\hline MHT-1C & 102706.8 & 48765.6 & 120 & 242.7 & 24.0 & 126.0 \\
\hline MHT-1C & 102706.8 & 48765.6 & 128 & 234.7 & 212.0 & 710.0 \\
\hline MHT-1C & 102706.8 & 48765.6 & 140 & 222.7 & 5.0 & 50.0 \\
\hline MHT-1C & 102706.8 & 48765.6 & 159 & 203.7 & 12.0 & 19.0 \\
\hline MHT-1C & 102706.8 & 48765.6 & 166 & 196.7 & 14.0 & 27.0 \\
\hline MHT-1C & 102706.8 & 48765.6 & 176 & 186.7 & 10.0 & 538.0 \\
\hline MHT-1C & 102706.8 & 48765.6 & 191 & 171.7 & - & 361.0 \\
\hline MHT-1C & 102706.8 & 48765.6 & 195 & 167.7 & - & 18.0 \\
\hline MHT-2C & 102747.1 & 48780.28 & 15 & 349.1 & 432.0 & 122.0 \\
\hline MHT-2C & 102747.1 & 48780.28 & 19 & 345.1 & 715.0 & 243.0 \\
\hline MHT-2C & 102747.1 & 48780.28 & 25 & 339.1 & 76.0 & 50.0 \\
\hline MHT-2C & 102747.1 & 48780.28 & 35 & 329.1 & $7,028.0$ & $4,948.0$ \\
\hline MHT-2C & 102747.1 & 48780.28 & 45 & 319.1 & 376.0 & 186.0 \\
\hline MHT-2C & 102747.1 & 48780.28 & 55 & 309.1 & $2,340.0$ & $1,838.0$ \\
\hline MHT-2C & 102747.1 & 48780.28 & 65 & 299.1 & - & - \\
\hline MHT-2C & 102747.1 & 48780.28 & 75 & 289.1 & 5.0 & 8.0 \\
\hline MHT-2C & 102747.1 & 48780.28 & 80 & 284.1 & - & - \\
\hline MHT-2C & 102747.1 & 48780.28 & 85 & 279.1 & 22.0 & 14.0 \\
\hline MHT-2C & 102747.1 & 48780.28 & 90 & 274.1 & 4.0 & 36.0 \\
\hline MHT-2C & 102747.1 & 48780.28 & 95 & 269.1 & 819.0 & $5,718.0$ \\
\hline MHT-2C & 102747.1 & 48780.28 & 99 & 265.1 & 622.0 & $11,221.0$ \\
\hline MHT-2C & 102747.1 & 48780.28 & 100 & 264.1 & - & 10.0 \\
\hline MHT-2C & 102747.1 & 48780.28 & 105 & 259.1 & - & 229.0 \\
\hline
\end{tabular}


WSRC-TR-2001-00077 February 2001

\begin{tabular}{|c|c|c|c|c|c|c|}
\hline ID & $\begin{array}{l}\text { SRS } \\
\text { North }\end{array}$ & $\begin{array}{l}\text { SRS } \\
\text { East }\end{array}$ & $\begin{array}{c}\text { Sample } \\
\text { Depth } \\
\mathrm{ft}\end{array}$ & $\begin{array}{c}\text { Sample } \\
\text { Elevation } \\
\mathrm{ft} \text { msl }\end{array}$ & $\begin{array}{c}\text { PCE } \\
(\mu \mathrm{g} / \mathrm{kg})\end{array}$ & $\begin{array}{c}\text { TCE } \\
(\mu \mathrm{g} / \mathrm{kg})\end{array}$ \\
\hline MHT-2C & 102747.1 & 48780.28 & 110 & 254.1 & 8.0 & 960.0 \\
\hline MHT-2C & 102747.1 & 48780.28 & 115 & 249.1 & 3.0 & 14.0 \\
\hline MHT-2C & 102747.1 & 48780.28 & 120 & 244.1 & - & - \\
\hline MHT-2C & 102747.1 & 48780.28 & 125 & 239.1 & 11.0 & 63.0 \\
\hline MHT-2C & 102747.1 & 48780.28 & 131 & 233.1 & 10.0 & 46.0 \\
\hline MHT-2C & 102747.1 & 48780.28 & 133 & 231.1 & 225.0 & 562.0 \\
\hline MHT-2C & 102747.1 & 48780.28 & 136 & 228.1 & 177.0 & 493.0 \\
\hline MHT-2C & 102747.1 & 48780.28 & 139 & 225.1 & 82.0 & 348.0 \\
\hline MHT-2C & 102747.1 & 48780.28 & 144 & 220.1 & 41.0 & 131.0 \\
\hline MHT-2C & 102747.1 & 48780.28 & 145 & 219.1 & 65.0 & 282.0 \\
\hline MHT-2C & 102747.1 & 48780.28 & 153 & 211.1 & 7.0 & 214.0 \\
\hline MHT-2C & 102747.1 & 48780.28 & 158 & 206.1 & - & 16.0 \\
\hline MHT-2C & 102747.1 & 48780.28 & 163 & 201.1 & 6.0 & 33.0 \\
\hline MHT-2C & 102747.1 & 48780.28 & 168 & 196.1 & 11.0 & 57.0 \\
\hline MHT-2C & 102747.1 & 48780.28 & 178 & 186.1 & - & 24.0 \\
\hline MHT-2C & 102747.1 & 48780.28 & 183 & 181.1 & - & 43.0 \\
\hline MHT-2C & 102747.1 & 48780.28 & 187 & 177.1 & - & $7,473.0$ \\
\hline MHT-2C & 102747.1 & 48780.28 & 190 & 174.1 & - & $1,162.0$ \\
\hline MHT-3C & 102704.3 & 48861.11 & 3 & 359.6 & - & - \\
\hline MHT-3C & 102704.3 & 48861.11 & 7 & 355.6 & - & - \\
\hline MHT-3C & 102704.3 & 48861.11 & 15 & 347.6 & 14.0 & 7.0 \\
\hline MHT-3C & 102704.3 & 48861.11 & 25 & 337.6 & 68.0 & 27.0 \\
\hline MHT-3C & 102704.3 & 48861.11 & 30 & 332.6 & - & - \\
\hline MHT-3C & 102704.3 & 48861.11 & 35 & 327.6 & 44.0 & 13.0 \\
\hline MHT-3C & 102704.3 & 48861.11 & 47 & 315.6 & - & - \\
\hline MHT-3C & 102704.3 & 48861.11 & 55 & 307.6 & 21.0 & 17.0 \\
\hline MHT-3C & 102704.3 & 48861.11 & 57 & 305.6 & 97.0 & 85.0 \\
\hline MHT-3C & 102704.3 & 48861.11 & 65 & 297.6 & 347.0 & 217.0 \\
\hline MHT-3C & 102704.3 & 48861.11 & 73 & 289.6 & - & - \\
\hline MHT-3C & 102704.3 & 48861.11 & 85 & 277.6 & 27.0 & 19.0 \\
\hline MHT-3C & 102704.3 & 48861.11 & 95 & 267.6 & 186.0 & 144.0 \\
\hline MHT-3C & 102704.3 & 48861.11 & 100 & 262.6 & 213.0 & 160.0 \\
\hline MHT-3C & 102704.3 & 48861.11 & 105 & 257.6 & 656.0 & $8,021.0$ \\
\hline MHT-3C & 102704.3 & 48861.11 & 115 & 247.6 & 75.0 & 793.0 \\
\hline MHT-3C & 102704.3 & 48861.11 & 120 & 242.6 & 25.0 & 183.0 \\
\hline MHT-3C & 102704.3 & 48861.11 & 126 & 236.6 & 290.0 & $1,235.0$ \\
\hline MHT-3C & 102704.3 & 48861.11 & 137 & 225.6 & 5.0 & 43.0 \\
\hline MHT-3C & 102704.3 & 48861.11 & 147 & 215.6 & 13.0 & 106.0 \\
\hline MHT-3C & 102704.3 & 48861.11 & 157 & 205.6 & 4.0 & 8.0 \\
\hline MHT-3C & 102704.3 & 48861.11 & 166 & 196.6 & 10.0 & 39.0 \\
\hline MHT-3C & 102704.3 & 48861.11 & 166 & 196.6 & 4.0 & 24.0 \\
\hline MHT-3C & 102704.3 & 48861.11 & 177 & 185.6 & - & 15.0 \\
\hline MHT-3C & 102704.3 & 48861.11 & 187 & 175.6 & - & $5,814.0$ \\
\hline MHT-4C & 102778.9 & 48863.53 & 3 & 364.4 & - & - \\
\hline MHT-4C & 102778.9 & 48863.53 & 5 & 362.4 & - & - \\
\hline MHT-4C & 102778.9 & 48863.53 & 15 & 352.4 & - & - \\
\hline
\end{tabular}


WSRC-TR-2001-00077 February 2001

\begin{tabular}{|c|c|c|c|c|c|c|}
\hline ID & $\begin{array}{l}\text { SRS } \\
\text { North }\end{array}$ & $\begin{array}{l}\text { SRS } \\
\text { East }\end{array}$ & $\begin{array}{l}\text { Sample } \\
\text { Depth } \\
\mathrm{ft}\end{array}$ & $\begin{array}{c}\text { Sample } \\
\text { Elevation } \\
\mathrm{ft} \mathrm{msl}\end{array}$ & $\begin{array}{c}\text { PCE } \\
(\mu \mathrm{g} / \mathrm{kg})\end{array}$ & $\begin{array}{c}\text { TCE } \\
(\mu \mathrm{g} / \mathrm{kg})\end{array}$ \\
\hline MHT-4C & 102778.9 & 48863.53 & 25 & 342.4 & - & - \\
\hline MHT-4C & 102778.9 & 48863.53 & 35 & 332.4 & 10.0 & 4.0 \\
\hline MHT-4C & 102778.9 & 48863.53 & 39 & 328.4 & 900.0 & 513.0 \\
\hline MHT-4C & 102778.9 & 48863.53 & 47 & 320.4 & 445.0 & 606.0 \\
\hline MHT-4C & 102778.9 & 48863.53 & 55 & 312.4 & 83.0 & 83.0 \\
\hline MHT-4C & 102778.9 & 48863.53 & 65 & 302.4 & 622.0 & 730.0 \\
\hline MHT-4C & 102778.9 & 48863.53 & 75 & 292.4 & - & - \\
\hline MHT-4C & 102778.9 & 48863.53 & 85 & 282.4 & - & - \\
\hline MHT-4C & 102778.9 & 48863.53 & 90 & 277.4 & - & - \\
\hline MHT-4C & 102778.9 & 48863.53 & 95 & 272.4 & $4,303.0$ & $11,964.0$ \\
\hline MHT-4C & 102778.9 & 48863.53 & 96 & 271.4 & 208.0 & 916.0 \\
\hline MHT-4C & 102778.9 & 48863.53 & 103 & 264.4 & 169.0 & 662.0 \\
\hline MHT-4C & 102778.9 & 48863.53 & 105 & 262.4 & $1,295.0$ & $8,202.0$ \\
\hline MHT-4C & 102778.9 & 48863.53 & 108 & 259.4 & 507.0 & $10,805.0$ \\
\hline MHT-4C & 102778.9 & 48863.53 & 109 & 258.4 & $1,813.0$ & $8,472.0$ \\
\hline MHT-4C & 102778.9 & 48863.53 & 115 & 252.4 & 911.0 & $3,048.0$ \\
\hline MHT-4C & 102778.9 & 48863.53 & 118 & 249.4 & 12.0 & 106.0 \\
\hline MHT-4C & 102778.9 & 48863.53 & 125 & 242.4 & 94.0 & 911.0 \\
\hline MHT-4C & 102778.9 & 48863.53 & 128 & 239.4 & - & 43.0 \\
\hline MHT-4C & 102778.9 & 48863.53 & 130 & 237.4 & 303.0 & $5,502.0$ \\
\hline MHT-4C & 102778.9 & 48863.53 & 134 & 233.4 & - & 5.0 \\
\hline MHT-4C & 102778.9 & 48863.53 & 138 & 229.4 & 27.0 & 402.0 \\
\hline MHT-4C & 102778.9 & 48863.53 & 140 & 227.4 & 3.0 & 62.0 \\
\hline MHT-4C & 102778.9 & 48863.53 & 142 & 225.4 & 80.0 & 645.0 \\
\hline MHT-4C & 102778.9 & 48863.53 & 145 & 222.4 & 27.0 & 971.0 \\
\hline MHT-4C & 102778.9 & 48863.53 & 152 & 215.4 & 37.0 & $1,288.0$ \\
\hline MHT-4C & 102778.9 & 48863.53 & 157 & 210.4 & 5.0 & 77.0 \\
\hline MHT-4C & 102778.9 & 48863.53 & 162 & 205.4 & 3.0 & 13.0 \\
\hline MHT-4C & 102778.9 & 48863.53 & 166 & 201.4 & 8.0 & 84.0 \\
\hline MHT-4C & 102778.9 & 48863.53 & 171 & 196.4 & 25.0 & 29.0 \\
\hline MHT-4C & 102778.9 & 48863.53 & 176 & 191.4 & 4.0 & 190.0 \\
\hline MHT-4C & 102778.9 & 48863.53 & 181 & 186.4 & 2.0 & 143.0 \\
\hline MHT-4C & 102778.9 & 48863.53 & 186 & 181.4 & - & $5,293.0$ \\
\hline MHT-4C & 102778.9 & 48863.53 & 190 & 177.4 & - & $7,812.0$ \\
\hline MHT-5C & 102725.1 & 48905.88 & 5 & 359.1 & - & - \\
\hline MHT-5C & 102725.1 & 48905.88 & 11 & 353.1 & - & - \\
\hline MHT-5C & 102725.1 & 48905.88 & 15 & 349.1 & - & - \\
\hline MHT-5C & 102725.1 & 48905.88 & 25 & 339.1 & - & - \\
\hline MHT-5C & 102725.1 & 48905.88 & 33 & 331.1 & 19.0 & 17.0 \\
\hline MHT-5C & 102725.1 & 48905.88 & 40 & 324.1 & 8.0 & 9.0 \\
\hline MHT-5C & 102725.1 & 48905.88 & 45 & 319.1 & 38.0 & 22.0 \\
\hline MHT-5C & 102725.1 & 48905.88 & 55 & 309.1 & 91.0 & 86.0 \\
\hline MHT-5C & 102725.1 & 48905.88 & 65 & 299.1 & 324.0 & 194.0 \\
\hline MHT-5C & 102725.1 & 48905.88 & 69 & 295.1 & - & - \\
\hline MHT-5C & 102725.1 & 48905.88 & 75 & 289.1 & - & - \\
\hline MHT-5C & 102725.1 & 48905.88 & 85 & 279.1 & 20.0 & 28.0 \\
\hline
\end{tabular}


WSRC-TR-2001-00077

February 2001

\begin{tabular}{|c|c|c|c|c|c|c|}
\hline ID & $\begin{array}{l}\text { SRS } \\
\text { North }\end{array}$ & $\begin{array}{l}\text { SRS } \\
\text { East }\end{array}$ & $\begin{array}{l}\text { Sample } \\
\text { Depth } \\
\mathrm{ft}\end{array}$ & $\begin{array}{c}\text { Sample } \\
\text { Elevation } \\
\mathrm{ft} \text { msl }\end{array}$ & $\begin{array}{c}\text { PCE } \\
(\mu \mathrm{g} / \mathrm{kg})\end{array}$ & $\begin{array}{c}\text { TCE } \\
(\mu \mathrm{g} / \mathrm{kg})\end{array}$ \\
\hline MHT-5C & 102725.1 & 48905.88 & 93 & 271.1 & 521.0 & $1,081.0$ \\
\hline MHT-5C & 102725.1 & 48905.88 & 101 & 263.1 & 133.0 & 140.0 \\
\hline MHT-5C & 102725.1 & 48905.88 & 108 & 256.1 & 515.0 & $7,235.0$ \\
\hline MHT-5C & 102725.1 & 48905.88 & 111 & 253.1 & 587.0 & $8,609.0$ \\
\hline MHT-5C & 102725.1 & 48905.88 & 117 & 247.1 & 985.0 & $5,775.0$ \\
\hline MHT-5C & 102725.1 & 48905.88 & 119 & 245.1 & 267.0 & $4,677.0$ \\
\hline MHT-5C & 102725.1 & 48905.88 & 126 & 238.1 & 63.0 & $1,002.0$ \\
\hline MHT-5C & 102725.1 & 48905.88 & 138 & 226.1 & 2.0 & 25.0 \\
\hline MHT-5C & 102725.1 & 48905.88 & 151 & 213.1 & 3.0 & 14.0 \\
\hline MHT-5C & 102725.1 & 48905.88 & 162 & 202.1 & 7.0 & 58.0 \\
\hline MHT-5C & 102725.1 & 48905.88 & 173 & 191.1 & 2.0 & 2.0 \\
\hline MHT-5C & 102725.1 & 48905.88 & 185 & 179.1 & - & 25.0 \\
\hline MHT-5C & 102725.1 & 48905.88 & 187 & 177.1 & - & $3,634.0$ \\
\hline MHT-5C & 102725.1 & 48905.88 & 189 & 175.1 & - & $11,653.0$ \\
\hline MHT-6C & 102810.8 & 48900.03 & 5 & 364.6 & - & - \\
\hline MHT-6C & 102810.8 & 48900.03 & 15 & 354.6 & - & - \\
\hline MHT-6C & 102810.8 & 48900.03 & 25 & 344.6 & - & - \\
\hline MHT-6C & 102810.8 & 48900.03 & 36 & 333.6 & - & - \\
\hline MHT-6C & 102810.8 & 48900.03 & 45 & 324.6 & 18.0 & 6.0 \\
\hline MHT-6C & 102810.8 & 48900.03 & 55 & 314.6 & 15.0 & 10.0 \\
\hline MHT-6C & 102810.8 & 48900.03 & 65 & 304.6 & 25.0 & 28.0 \\
\hline MHT-6C & 102810.8 & 48900.03 & 75 & 294.6 & 9.0 & 38.0 \\
\hline MHT-6C & 102810.8 & 48900.03 & 85 & 284.6 & - & 10.0 \\
\hline MHT-6C & 102810.8 & 48900.03 & 91 & 278.6 & - & - \\
\hline MHT-6C & 102810.8 & 48900.03 & 95 & 274.6 & - & - \\
\hline MHT-6C & 102810.8 & 48900.03 & 101 & 268.6 & 27.0 & $3,108.0$ \\
\hline MHT-6C & 102810.8 & 48900.03 & 103 & 266.6 & $2,966.0$ & $16,323.0$ \\
\hline MHT-6C & 102810.8 & 48900.03 & 107 & 262.6 & 608.0 & $3,443.0$ \\
\hline MHT-6C & 102810.8 & 48900.03 & 111 & 258.6 & 644.0 & $8,537.0$ \\
\hline MHT-6C & 102810.8 & 48900.03 & 115 & 254.6 & 155.0 & $5,121.0$ \\
\hline MHT-6C & 102810.8 & 48900.03 & 121 & 248.6 & 39.0 & $1,911.0$ \\
\hline MHT-6C & 102810.8 & 48900.03 & 125 & 244.6 & 5.0 & 215.0 \\
\hline MHT-6C & 102810.8 & 48900.03 & 131 & 238.6 & 322.0 & $9,126.0$ \\
\hline MHT-6C & 102810.8 & 48900.03 & 135 & 234.6 & - & - \\
\hline MHT-6C & 102810.8 & 48900.03 & 137 & 232.6 & 9.0 & 601.0 \\
\hline MHT-6C & 102810.8 & 48900.03 & 141 & 228.6 & 7.0 & 156.0 \\
\hline MHT-6C & 102810.8 & 48900.03 & 145 & 224.6 & 4.0 & 386.0 \\
\hline MHT-6C & 102810.8 & 48900.03 & 151 & 218.6 & 4.0 & 313.0 \\
\hline MHT-6C & 102810.8 & 48900.03 & 155 & 214.6 & 35.0 & $1,030.0$ \\
\hline MHT-6C & 102810.8 & 48900.03 & 160 & 209.6 & 7.0 & 170.0 \\
\hline MHT-6C & 102810.8 & 48900.03 & 165 & 204.6 & - & - \\
\hline MHT-6C & 102810.8 & 48900.03 & 170 & 199.6 & 34.0 & 36.0 \\
\hline MHT-6C & 102810.8 & 48900.03 & 175 & 194.6 & 10.0 & 27.0 \\
\hline MHT-6C & 102810.8 & 48900.03 & 180 & 189.6 & 26.0 & $1,475.0$ \\
\hline MHT-6C & 102810.8 & 48900.03 & 181 & 188.6 & 40.0 & $2,110.0$ \\
\hline MHT-6C & 102810.8 & 48900.03 & 185 & 184.6 & - & 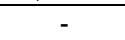 \\
\hline
\end{tabular}


WSRC-TR-2001-00077 February 2001

\begin{tabular}{|c|c|c|c|c|c|c|}
\hline ID & $\begin{array}{l}\text { SRS } \\
\text { North }\end{array}$ & $\begin{array}{l}\text { SRS } \\
\text { East }\end{array}$ & $\begin{array}{c}\text { Sample } \\
\text { Depth } \\
\mathrm{ft}\end{array}$ & $\begin{array}{c}\text { Sample } \\
\text { Elevation } \\
\mathrm{ft} \text { msl }\end{array}$ & $\begin{array}{c}\text { PCE } \\
(\mu \mathrm{g} / \mathrm{kg})\end{array}$ & $\begin{array}{c}\text { TCE } \\
(\mu \mathrm{g} / \mathrm{kg})\end{array}$ \\
\hline MHT-6C & 102810.8 & 48900.03 & 187.5 & 182.1 & - & - \\
\hline MHT-6C & 102810.8 & 48900.03 & 187.5 & 182.1 & - & - \\
\hline MHT-6C & 102810.8 & 48900.03 & 187.5 & 182.1 & - & 5.0 \\
\hline MHT-6C & 102810.8 & 48900.03 & 188 & 181.6 & - & $1,842.0$ \\
\hline MHT-6C & 102810.8 & 48900.03 & 189 & 180.6 & - & 27.0 \\
\hline MHT-7C & 102788.9 & 48977.48 & 5 & 363 & - & - \\
\hline MHT-7C & 102788.9 & 48977.48 & 15 & 353 & - & - \\
\hline MHT-7C & 102788.9 & 48977.48 & 25 & 343 & - & - \\
\hline MHT-7C & 102788.9 & 48977.48 & 35 & 333 & - & - \\
\hline MHT-7C & 102788.9 & 48977.48 & 45 & 323 & - & - \\
\hline MHT-7C & 102788.9 & 48977.48 & 55 & 313 & - & - \\
\hline MHT-7C & 102788.9 & 48977.48 & 71 & 297 & 11.0 & 17.0 \\
\hline MHT-7C & 102788.9 & 48977.48 & 75 & 293 & - & 4.0 \\
\hline MHT-7C & 102788.9 & 48977.48 & 85 & 283 & - & - \\
\hline MHT-7C & 102788.9 & 48977.48 & 93 & 275 & 172.0 & 992.0 \\
\hline MHT-7C & 102788.9 & 48977.48 & 105 & 263 & - & 204.0 \\
\hline MHT-7C & 102788.9 & 48977.48 & 115 & 253 & 12.0 & 208.0 \\
\hline MHT-7C & 102788.9 & 48977.48 & 119 & 249 & - & 32.0 \\
\hline MHT-7C & 102788.9 & 48977.48 & 131 & 237 & - & 9.0 \\
\hline MHT-7C & 102788.9 & 48977.48 & 141 & 227 & - & 15.0 \\
\hline MHT-7C & 102788.9 & 48977.48 & 143 & 225 & - & 4.0 \\
\hline MHT-7C & 102788.9 & 48977.48 & 145 & 223 & 4.0 & 11.0 \\
\hline MHT-7C & 102788.9 & 48977.48 & 155 & 213 & - & 6.0 \\
\hline MHT-7C & 102788.9 & 48977.48 & 177 & 191 & - & 156.0 \\
\hline MHT-7C & 102788.9 & 48977.48 & 179 & 189 & - & $1,260.0$ \\
\hline MHT-7C & 102788.9 & 48977.48 & 181 & 187 & - & 7.0 \\
\hline MHT-7C & 102788.9 & 48977.48 & 189 & 179 & - & 480.0 \\
\hline MHT-7C & 102788.9 & 48977.48 & 191 & 177 & - & 4.0 \\
\hline MHT-8C & 102880.7 & 48970.24 & 5 & 364.3 & - & - \\
\hline MHT-8C & 102880.7 & 48970.24 & 15 & 354.3 & - & - \\
\hline MHT-8C & 102880.7 & 48970.24 & 25 & 344.3 & - & - \\
\hline MHT-8C & 102880.7 & 48970.24 & 35 & 334.3 & - & - \\
\hline MHT-8C & 102880.7 & 48970.24 & 45 & 324.3 & - & 10.0 \\
\hline MHT-8C & 102880.7 & 48970.24 & 55 & 314.3 & 12.0 & 95.0 \\
\hline MHT-8C & 102880.7 & 48970.24 & 65 & 304.3 & 51.0 & 381.0 \\
\hline MHT-8C & 102880.7 & 48970.24 & 75 & 294.3 & 64.0 & 633.0 \\
\hline MHT-8C & 102880.7 & 48970.24 & 85 & 284.3 & 22.0 & 271.0 \\
\hline MHT-8C & 102880.7 & 48970.24 & 95 & 274.3 & 6.0 & 101.0 \\
\hline MHT-8C & 102880.7 & 48970.24 & 105 & 264.3 & - & - \\
\hline MHT-8C & 102880.7 & 48970.24 & 113 & 256.3 & 4.0 & $1,250.0$ \\
\hline MHT-8C & 102880.7 & 48970.24 & 124 & 245.3 & 109.0 & $3,017.0$ \\
\hline MHT-8C & 102880.7 & 48970.24 & 129 & 240.3 & 17.0 & $1,160.0$ \\
\hline MHT-8C & 102880.7 & 48970.24 & 134 & 235.3 & 3.0 & 154.0 \\
\hline MHT-8C & 102880.7 & 48970.24 & 139 & 230.3 & 2.0 & 196.0 \\
\hline MHT-8C & 102880.7 & 48970.24 & 144 & 225.3 & 10.0 & 23.0 \\
\hline MHT-8C & 102880.7 & 48970.24 & 149 & 220.3 & 27.0 & 360.0 \\
\hline
\end{tabular}


WSRC-TR-2001-00077

February 2001

\begin{tabular}{|c|c|c|c|c|c|c|}
\hline ID & $\begin{array}{l}\text { SRS } \\
\text { North }\end{array}$ & $\begin{array}{l}\text { SRS } \\
\text { East }\end{array}$ & $\begin{array}{l}\text { Sample } \\
\text { Depth } \\
\mathrm{ft}\end{array}$ & $\begin{array}{c}\text { Sample } \\
\text { Elevation } \\
\mathrm{ft} \text { msl }\end{array}$ & $\begin{array}{c}\text { PCE } \\
(\mu \mathrm{g} / \mathrm{kg})\end{array}$ & $\begin{array}{c}\text { TCE } \\
(\mu \mathrm{g} / \mathrm{kg})\end{array}$ \\
\hline MHT-8C & 102880.7 & 48970.24 & 154 & 215.3 & - & 52.0 \\
\hline MHT-8C & 102880.7 & 48970.24 & 159 & 210.3 & - & 25.0 \\
\hline MHT-8C & 102880.7 & 48970.24 & 164 & 205.3 & 4.0 & 15.0 \\
\hline MHT-8C & 102880.7 & 48970.24 & 169 & 200.3 & 4.0 & 125.0 \\
\hline MHT-8C & 102880.7 & 48970.24 & 174 & 195.3 & - & 233.0 \\
\hline MHT-8C & 102880.7 & 48970.24 & 179 & 190.3 & - & - \\
\hline MHT-8C & 102880.7 & 48970.24 & 184 & 185.3 & - & 267.0 \\
\hline MHT-8C & 102880.7 & 48970.24 & 189 & 180.3 & - & 148.0 \\
\hline MHT-9C & 102814.4 & 49015.58 & 5 & 362.7 & - & - \\
\hline MHT-9C & 102814.4 & 49015.58 & 15 & 352.7 & - & - \\
\hline MHT-9C & 102814.4 & 49015.58 & 25 & 342.7 & - & - \\
\hline MHT-9C & 102814.4 & 49015.58 & 37 & 330.7 & - & - \\
\hline MHT-9C & 102814.4 & 49015.58 & 45 & 322.7 & - & 3.0 \\
\hline MHT-9C & 102814.4 & 49015.58 & 55 & 312.7 & - & - \\
\hline MHT-9C & 102814.4 & 49015.58 & 65 & 302.7 & - & 5.0 \\
\hline MHT-9C & 102814.4 & 49015.58 & 75 & 292.7 & - & 5.0 \\
\hline MHT-9C & 102814.4 & 49015.58 & 85 & 282.7 & - & - \\
\hline MHT-9C & 102814.4 & 49015.58 & 89 & 278.7 & 39.0 & 729.0 \\
\hline MHT-9C & 102814.4 & 49015.58 & 95 & 272.7 & - & - \\
\hline MHT-9C & 102814.4 & 49015.58 & 101 & 266.7 & - & 177.0 \\
\hline MHT-9C & 102814.4 & 49015.58 & 105 & 262.7 & - & 126.0 \\
\hline MHT-9C & 102814.4 & 49015.58 & 107 & 260.7 & - & - \\
\hline MHT-9C & 102814.4 & 49015.58 & 109 & 258.7 & 93.0 & $1,528.0$ \\
\hline MHT-9C & 102814.4 & 49015.58 & 115 & 252.7 & - & 6.0 \\
\hline MHT-9C & 102814.4 & 49015.58 & 130 & 237.7 & 44.0 & 889.0 \\
\hline MHT-9C & 102814.4 & 49015.58 & 131 & 236.7 & 11.0 & 256.0 \\
\hline MHT-9C & 102814.4 & 49015.58 & 133 & 234.7 & 14.0 & 305.0 \\
\hline MHT-9C & 102814.4 & 49015.58 & 141 & 226.7 & - & 17.0 \\
\hline MHT-9C & 102814.4 & 49015.58 & 143 & 224.7 & 6.0 & 12.0 \\
\hline MHT-9C & 102814.4 & 49015.58 & 145 & 222.7 & 5.0 & 8.0 \\
\hline MHT-9C & 102814.4 & 49015.58 & 153 & 214.7 & - & - \\
\hline MHT-9C & 102814.4 & 49015.58 & 155 & 212.7 & - & - \\
\hline MHT-9C & 102814.4 & 49015.58 & 157 & 210.7 & - & - \\
\hline MHT-9C & 102814.4 & 49015.58 & 165 & 202.7 & - & 28.0 \\
\hline MHT-9C & 102814.4 & 49015.58 & 167 & 200.7 & 5.0 & 176.0 \\
\hline MHT-9C & 102814.4 & 49015.58 & 179 & 188.7 & - & - \\
\hline MHT-9C & 102814.4 & 49015.58 & 181 & 186.7 & - & 48.0 \\
\hline MHT-9C & 102814.4 & 49015.58 & 189 & 178.7 & - & $1,466.0$ \\
\hline MHT-10C & 102892.3 & 49011.57 & 7 & 361.9 & - & - \\
\hline MHT-10C & 102892.3 & 49011.57 & 15 & 353.9 & - & - \\
\hline MHT-10C & 102892.3 & 49011.57 & 25 & 343.9 & - & - \\
\hline MHT-10C & 102892.3 & 49011.57 & 35 & 333.9 & - & - \\
\hline MHT-10C & 102892.3 & 49011.57 & 45 & 323.9 & - & 12.0 \\
\hline MHT-10C & 102892.3 & 49011.57 & 55 & 313.9 & 5.0 & 42.0 \\
\hline MHT-10C & 102892.3 & 49011.57 & 65 & 303.9 & - & 49.0 \\
\hline MHT-10C & 102892.3 & 49011.57 & 75 & 293.9 & - & 37.0 \\
\hline
\end{tabular}


WSRC-TR-2001-00077 February 2001

\begin{tabular}{|c|c|c|c|c|c|c|}
\hline ID & $\begin{array}{c}\text { SRS } \\
\text { North }\end{array}$ & $\begin{array}{l}\text { SRS } \\
\text { East }\end{array}$ & $\begin{array}{c}\text { Sample } \\
\text { Depth } \\
\mathrm{ft}\end{array}$ & $\begin{array}{c}\text { Sample } \\
\text { Elevation } \\
\mathrm{ft} \text { msl }\end{array}$ & $\begin{array}{c}\text { PCE } \\
(\mu \mathrm{g} / \mathrm{kg})\end{array}$ & $\begin{array}{c}\text { TCE } \\
(\mu \mathrm{g} / \mathrm{kg})\end{array}$ \\
\hline MHT-10C & 102892.3 & 49011.57 & 87 & 281.9 & - & - \\
\hline MHT-10C & 102892.3 & 49011.57 & 95 & 273.9 & 26.0 & 390.0 \\
\hline MHT-10C & 102892.3 & 49011.57 & 105 & 263.9 & - & 37.0 \\
\hline MHT-10C & 102892.3 & 49011.57 & 111 & 257.9 & 23.0 & $2,771.0$ \\
\hline MHT-10C & 102892.3 & 49011.57 & 120 & 248.9 & - & 27.0 \\
\hline MHT-10C & 102892.3 & 49011.57 & 128 & 240.9 & 34.0 & $1,971.0$ \\
\hline MHT-10C & 102892.3 & 49011.57 & 131 & 237.9 & - & 333.0 \\
\hline MHT-10C & 102892.3 & 49011.57 & 141 & 227.9 & 3.0 & 240.0 \\
\hline MHT-10C & 102892.3 & 49011.57 & 148 & 220.9 & 12.0 & 16.0 \\
\hline MHT-10C & 102892.3 & 49011.57 & 157 & 211.9 & - & 95.0 \\
\hline MHT-10C & 102892.3 & 49011.57 & 167 & 201.9 & 11.0 & 25.0 \\
\hline MHT-10C & 102892.3 & 49011.57 & 170 & 198.9 & - & 117.0 \\
\hline MHT-10C & 102892.3 & 49011.57 & 175 & 193.9 & - & - \\
\hline MHT-10C & 102892.3 & 49011.57 & 180 & 188.9 & - & 36.0 \\
\hline MHT-10C & 102892.3 & 49011.57 & 185 & 183.9 & - & 304.0 \\
\hline MHT-10C & 102892.3 & 49011.57 & 190 & 178.9 & - & 525.0 \\
\hline MHT-15C & 102520.7 & 48741.9 & 5 & 352.7 & - & - \\
\hline MHT-15C & 102520.7 & 48741.9 & 10 & 347.7 & - & - \\
\hline MHT-15C & 102520.7 & 48741.9 & 15 & 342.7 & - & - \\
\hline MHT-15C & 102520.7 & 48741.9 & 19 & 338.7 & 204.7 & 1.0 \\
\hline MHT-15C & 102520.7 & 48741.9 & 20 & 337.7 & 581.9 & 75.3 \\
\hline MHT-15C & 102520.7 & 48741.9 & 25 & 332.7 & 35.7 & - \\
\hline MHT-15C & 102520.7 & 48741.9 & 30 & 327.7 & 40.7 & - \\
\hline MHT-15C & 102520.7 & 48741.9 & 35 & 322.7 & 733.0 & 577.1 \\
\hline MHT-15C & 102520.7 & 48741.9 & 38 & 319.7 & 646.8 & $1,066.2$ \\
\hline MHT-15C & 102520.7 & 48741.9 & 40 & 317.7 & 324.2 & 300.4 \\
\hline MHT-15C & 102520.7 & 48741.9 & 45 & 312.7 & 465.0 & 669.7 \\
\hline MHT-15C & 102520.7 & 48741.9 & 50 & 307.7 & 332.8 & 152.0 \\
\hline MHT-15C & 102520.7 & 48741.9 & 55 & 302.7 & 557.8 & $1,144.7$ \\
\hline MHT-15C & 102520.7 & 48741.9 & 60 & 297.7 & 733.8 & $1,144.2$ \\
\hline MHT-15C & 102520.7 & 48741.9 & 65 & 292.7 & 698.4 & $1,329.2$ \\
\hline MHT-15C & 102520.7 & 48741.9 & 70 & 287.7 & - & - \\
\hline MHT-15C & 102520.7 & 48741.9 & 75 & 282.7 & - & - \\
\hline MHT-15C & 102520.7 & 48741.9 & 80 & 277.7 & - & - \\
\hline MHT-15C & 102520.7 & 48741.9 & 85 & 272.7 & 797.8 & $1,847.6$ \\
\hline MHT-15C & 102520.7 & 48741.9 & 90 & 267.7 & 742.3 & $2,131.9$ \\
\hline MHT-15C & 102520.7 & 48741.9 & 95 & 262.7 & 498.5 & $1,030.6$ \\
\hline MHT-15C & 102520.7 & 48741.9 & 100 & 257.7 & 246.8 & 89.1 \\
\hline MHT-15C & 102520.7 & 48741.9 & 105 & 252.7 & - & 1.3 \\
\hline MHT-15C & 102520.7 & 48741.9 & 110 & 247.7 & - & - \\
\hline MHT-15C & 102520.7 & 48741.9 & 115 & 242.7 & - & 114.4 \\
\hline MHT-15C & 102520.7 & 48741.9 & 120 & 237.7 & - & - \\
\hline MHT-15C & 102520.7 & 48741.9 & 125 & 232.7 & - & - \\
\hline MHT-15C & 102520.7 & 48741.9 & 130 & 227.7 & - & 64.8 \\
\hline MHT-15C & 102520.7 & 48741.9 & 141 & 216.7 & 178.5 & 257.1 \\
\hline MHT-15C & 102520.7 & 48741.9 & 146 & 211.7 & - & 51.1 \\
\hline
\end{tabular}


WSRC-TR-2001-00077

February 2001

\begin{tabular}{|c|c|c|c|c|c|c|}
\hline ID & $\begin{array}{l}\text { SRS } \\
\text { North }\end{array}$ & $\begin{array}{l}\text { SRS } \\
\text { East }\end{array}$ & $\begin{array}{l}\text { Sample } \\
\text { Depth } \\
\mathrm{ft}\end{array}$ & $\begin{array}{c}\text { Sample } \\
\text { Elevation } \\
\mathrm{ft} \text { msl }\end{array}$ & $\begin{array}{c}\text { PCE } \\
(\mu \mathrm{g} / \mathrm{kg})\end{array}$ & $\begin{array}{c}\text { TCE } \\
(\mu \mathrm{g} / \mathrm{kg})\end{array}$ \\
\hline MHT-15C & 102520.7 & 48741.9 & 151 & 206.7 & - & 19.9 \\
\hline MHT-15C & 102520.7 & 48741.9 & 161 & 196.7 & 40.5 & 39.9 \\
\hline MHT-15C & 102520.7 & 48741.9 & 171 & 186.7 & - & - \\
\hline MHT-15C & 102520.7 & 48741.9 & 179 & 178.7 & - & $1,449.0$ \\
\hline MHT-15C & 102520.7 & 48741.9 & 181 & 176.7 & - & $1,627.6$ \\
\hline MHT-15C & 102520.7 & 48741.9 & 186 & 171.7 & - & $1,188.3$ \\
\hline MHT-15C & 102520.7 & 48741.9 & 191 & 166.7 & - & 43.1 \\
\hline MHT-15C & 102520.7 & 48741.9 & 196 & 161.7 & - & - \\
\hline MHT-15C & 102520.7 & 48741.9 & 198 & 159.7 & - & 342.5 \\
\hline MHT-16C & 102430.9 & 48672.8 & 5 & 354.5 & - & - \\
\hline MHT-16C & 102430.9 & 48672.8 & 10 & 349.5 & - & - \\
\hline MHT-16C & 102430.9 & 48672.8 & 15 & 344.5 & - & - \\
\hline MHT-16C & 102430.9 & 48672.8 & 20 & 339.5 & 342.7 & 68.9 \\
\hline MHT-16C & 102430.9 & 48672.8 & 25 & 334.5 & 136.9 & - \\
\hline MHT-16C & 102430.9 & 48672.8 & 30 & 329.5 & 22.1 & - \\
\hline MHT-16C & 102430.9 & 48672.8 & 35 & 324.5 & 334.5 & 12.6 \\
\hline MHT-16C & 102430.9 & 48672.8 & 40 & 319.5 & 706.7 & $1,429.4$ \\
\hline MHT-16C & 102430.9 & 48672.8 & 45 & 314.5 & 815.5 & $2,204.5$ \\
\hline MHT-16C & 102430.9 & 48672.8 & 50 & 309.5 & - & 14.1 \\
\hline MHT-16C & 102430.9 & 48672.8 & 55 & 304.5 & 48.2 & 146.3 \\
\hline MHT-16C & 102430.9 & 48672.8 & 60 & 299.5 & 445.7 & $1,123.3$ \\
\hline MHT-16C & 102430.9 & 48672.8 & 65 & 294.5 & 255.4 & 421.8 \\
\hline MHT-16C & 102430.9 & 48672.8 & 70 & 289.5 & - & - \\
\hline MHT-16C & 102430.9 & 48672.8 & 75 & 284.5 & - & - \\
\hline MHT-16C & 102430.9 & 48672.8 & 80 & 279.5 & - & - \\
\hline MHT-16C & 102430.9 & 48672.8 & 85 & 274.5 & - & - \\
\hline MHT-16C & 102430.9 & 48672.8 & 90 & 269.5 & 400.2 & $1,156.5$ \\
\hline MHT-16C & 102430.9 & 48672.8 & 95 & 264.5 & 590.1 & $1,706.1$ \\
\hline MHT-16C & 102430.9 & 48672.8 & 100 & 259.5 & 119.5 & 267.3 \\
\hline MHT-16C & 102430.9 & 48672.8 & 105 & 254.5 & - & 5.5 \\
\hline MHT-16C & 102430.9 & 48672.8 & 110 & 249.5 & - & - \\
\hline MHT-16C & 102430.9 & 48672.8 & 115 & 244.5 & 605.9 & $1,480.9$ \\
\hline MHT-16C & 102430.9 & 48672.8 & 120 & 239.5 & 77.0 & 129.3 \\
\hline MHT-16C & 102430.9 & 48672.8 & 125 & 234.5 & 679.6 & $1,559.5$ \\
\hline MHT-16C & 102430.9 & 48672.8 & 130 & 229.5 & 408.2 & 573.4 \\
\hline MHT-16C & 102430.9 & 48672.8 & 134 & 225.5 & 385.8 & 447.1 \\
\hline MHT-16C & 102430.9 & 48672.8 & 140 & 219.5 & 1.0 & 64.3 \\
\hline MHT-16C & 102430.9 & 48672.8 & 145 & 214.5 & 785.2 & $2,016.8$ \\
\hline MHT-16C & 102430.9 & 48672.8 & 150 & 209.5 & 646.2 & $1,701.2$ \\
\hline MHT-16C & 102430.9 & 48672.8 & 155 & 204.5 & 424.3 & 255.5 \\
\hline MHT-16C & 102430.9 & 48672.8 & 160 & 199.5 & 854.5 & 74.7 \\
\hline MHT-16C & 102430.9 & 48672.8 & 165 & 194.5 & 979.0 & $2,631.2$ \\
\hline MHT-16C & 102430.9 & 48672.8 & 170 & 189.5 & 931.5 & $2,431.3$ \\
\hline MHT-16C & 102430.9 & 48672.8 & 175 & 184.5 & 709.8 & 946.1 \\
\hline MHT-16C & 102430.9 & 48672.8 & 180 & 179.5 & $1,429.4$ & $3,102.5$ \\
\hline MHT-16C & 102430.9 & 48672.8 & 185 & 174.5 & 737.2 & $2,025.6$ \\
\hline
\end{tabular}


WSRC-TR-2001-00077

February 2001

\begin{tabular}{|c|c|c|c|c|c|c|}
\hline ID & $\begin{array}{l}\text { SRS } \\
\text { North }\end{array}$ & $\begin{array}{l}\text { SRS } \\
\text { East }\end{array}$ & $\begin{array}{l}\text { Sample } \\
\text { Depth } \\
\mathrm{ft}\end{array}$ & $\begin{array}{c}\text { Sample } \\
\text { Elevation } \\
\mathrm{ft} \text { msl }\end{array}$ & $\begin{array}{c}\text { PCE } \\
(\mu \mathrm{g} / \mathrm{kg})\end{array}$ & $\begin{array}{c}\text { TCE } \\
(\mu \mathrm{g} / \mathrm{kg})\end{array}$ \\
\hline MHT-16C & 102430.9 & 48672.8 & 190 & 169.5 & 741.0 & $1,271.5$ \\
\hline MHT-16C & 102430.9 & 48672.8 & 195 & 164.5 & 636.6 & $1,622.6$ \\
\hline MHT-16C & 102430.9 & 48672.8 & 199 & 160.5 & 244.0 & 323.5 \\
\hline MHT-17C & 102394.6 & 48706.9 & 5 & 352.7 & - & - \\
\hline MHT-17C & 102394.6 & 48706.9 & 10 & 347.7 & - & - \\
\hline MHT-17C & 102394.6 & 48706.9 & 15 & 342.7 & - & - \\
\hline MHT-17C & 102394.6 & 48706.9 & 20 & 337.7 & - & - \\
\hline MHT-17C & 102394.6 & 48706.9 & 25 & 332.7 & 2.5 & - \\
\hline MHT-17C & 102394.6 & 48706.9 & 30 & 327.7 & 186.5 & 7.9 \\
\hline MHT-17C & 102394.6 & 48706.9 & 35 & 322.7 & 484.4 & 81.2 \\
\hline MHT-17C & 102394.6 & 48706.9 & 40 & 317.7 & - & - \\
\hline MHT-17C & 102394.6 & 48706.9 & 45 & 312.7 & 83.7 & 114.8 \\
\hline MHT-17C & 102394.6 & 48706.9 & 50 & 307.7 & - & - \\
\hline MHT-17C & 102394.6 & 48706.9 & 55 & 302.7 & 210.4 & 191.0 \\
\hline MHT-17C & 102394.6 & 48706.9 & 60 & 297.7 & 594.9 & $1,377.2$ \\
\hline MHT-17C & 102394.6 & 48706.9 & 65 & 292.7 & 458.3 & $1,104.1$ \\
\hline MHT-17C & 102394.6 & 48706.9 & 70 & 287.7 & 101.3 & 67.1 \\
\hline MHT-17C & 102394.6 & 48706.9 & 75 & 282.7 & - & - \\
\hline MHT-17C & 102394.6 & 48706.9 & 80 & 277.7 & - & - \\
\hline MHT-17C & 102394.6 & 48706.9 & 85 & 272.7 & - & - \\
\hline MHT-17C & 102394.6 & 48706.9 & 90 & 267.7 & 412.0 & $1,506.0$ \\
\hline MHT-17C & 102394.6 & 48706.9 & 95 & 262.7 & 508.5 & $1,328.9$ \\
\hline MHT-17C & 102394.6 & 48706.9 & 100 & 257.7 & 535.9 & $1,248.1$ \\
\hline MHT-17C & 102394.6 & 48706.9 & 105 & 252.7 & - & - \\
\hline MHT-17C & 102394.6 & 48706.9 & 110 & 247.7 & 718.1 & $1,682.8$ \\
\hline MHT-17C & 102394.6 & 48706.9 & 115 & 242.7 & 466.0 & 629.0 \\
\hline MHT-17C & 102394.6 & 48706.9 & 120 & 237.7 & 104.0 & 323.1 \\
\hline MHT-17C & 102394.6 & 48706.9 & 125 & 232.7 & 96.8 & 409.0 \\
\hline MHT-17C & 102394.6 & 48706.9 & 130 & 227.7 & 27.0 & 43.2 \\
\hline MHT-17C & 102394.6 & 48706.9 & 135 & 222.7 & 408.1 & 315.9 \\
\hline MHT-17C & 102394.6 & 48706.9 & 140 & 217.7 & 259.3 & 154.5 \\
\hline MHT-17C & 102394.6 & 48706.9 & 145 & 212.7 & - & 819.5 \\
\hline MHT-17C & 102394.6 & 48706.9 & 150 & 207.7 & - & $1,142.9$ \\
\hline MHT-17C & 102394.6 & 48706.9 & 155 & 202.7 & - & 11.1 \\
\hline MHT-17C & 102394.6 & 48706.9 & 160 & 197.7 & - & - \\
\hline MHT-17C & 102394.6 & 48706.9 & 165 & 192.7 & 1.6 & 57.1 \\
\hline MHT-17C & 102394.6 & 48706.9 & 170 & 187.7 & - & - \\
\hline MHT-17C & 102394.6 & 48706.9 & 175 & 182.7 & - & 222.6 \\
\hline MHT-17C & 102394.6 & 48706.9 & 178 & 179.7 & 299.5 & $2,313.2$ \\
\hline MHT-17C & 102394.6 & 48706.9 & 180 & 177.7 & 46.4 & $1,905.5$ \\
\hline MHT-17C & 102394.6 & 48706.9 & 185 & 172.7 & 348.0 & $1,561.6$ \\
\hline MHT-17C & 102394.6 & 48706.9 & 190 & 167.7 & - & $1,533.4$ \\
\hline MHT-17C & 102394.6 & 48706.9 & 195 & 162.7 & - & 80.3 \\
\hline MHT-17C & 102394.6 & 48706.9 & 200 & 157.7 & - & 336.3 \\
\hline MHT-18C & 102486.1 & 48650.9 & 5 & 355.3 & - & - \\
\hline MHT-18C & 102486.1 & 48650.9 & 10 & 350.3 & - & - \\
\hline
\end{tabular}


WSRC-TR-2001-00077 February 2001

\begin{tabular}{|c|c|c|c|c|c|c|}
\hline ID & $\begin{array}{l}\text { SRS } \\
\text { North }\end{array}$ & $\begin{array}{l}\text { SRS } \\
\text { East }\end{array}$ & $\begin{array}{c}\text { Sample } \\
\text { Depth } \\
\mathrm{ft}\end{array}$ & $\begin{array}{c}\text { Sample } \\
\text { Elevation } \\
\mathrm{ft} \text { msl }\end{array}$ & $\begin{array}{c}\text { PCE } \\
(\mu \mathrm{g} / \mathrm{kg})\end{array}$ & $\begin{array}{c}\text { TCE } \\
(\mu \mathrm{g} / \mathrm{kg})\end{array}$ \\
\hline MHT-18C & 102486.1 & 48650.9 & 15 & 345.3 & - & - \\
\hline MHT-18C & 102486.1 & 48650.9 & 20 & 340.3 & - & - \\
\hline MHT-18C & 102486.1 & 48650.9 & 25 & 335.3 & - & - \\
\hline MHT-18C & 102486.1 & 48650.9 & 30 & 330.3 & - & - \\
\hline MHT-18C & 102486.1 & 48650.9 & 35 & 325.3 & - & - \\
\hline MHT-18C & 102486.1 & 48650.9 & 40 & 320.3 & 20.8 & 27.4 \\
\hline MHT-18C & 102486.1 & 48650.9 & 45 & 315.3 & 131.1 & 120.9 \\
\hline MHT-18C & 102486.1 & 48650.9 & 50 & 310.3 & - & 3.7 \\
\hline MHT-18C & 102486.1 & 48650.9 & 55 & 305.3 & - & 9.8 \\
\hline MHT-18C & 102486.1 & 48650.9 & 60 & 300.3 & - & 1.3 \\
\hline MHT-18C & 102486.1 & 48650.9 & 65 & 295.3 & 47.5 & 64.2 \\
\hline MHT-18C & 102486.1 & 48650.9 & 70 & 290.3 & - & 5.8 \\
\hline MHT-18C & 102486.1 & 48650.9 & 75 & 285.3 & - & - \\
\hline MHT-18C & 102486.1 & 48650.9 & 80 & 280.3 & - & - \\
\hline MHT-18C & 102486.1 & 48650.9 & 85 & 275.3 & - & - \\
\hline MHT-18C & 102486.1 & 48650.9 & 90 & 270.3 & 711.2 & $1,503.7$ \\
\hline MHT-18C & 102486.1 & 48650.9 & 93 & 267.3 & 674.6 & $1,187.3$ \\
\hline MHT-18C & 102486.1 & 48650.9 & 95 & 265.3 & 656.4 & $1,219.5$ \\
\hline MHT-18C & 102486.1 & 48650.9 & 100 & 260.3 & - & - \\
\hline MHT-18C & 102486.1 & 48650.9 & 105 & 255.3 & - & 19.5 \\
\hline MHT-18C & 102486.1 & 48650.9 & 110 & 250.3 & - & - \\
\hline MHT-18C & 102486.1 & 48650.9 & 115 & 245.3 & - & - \\
\hline MHT-18C & 102486.1 & 48650.9 & 120 & 240.3 & - & - \\
\hline MHT-18C & 102486.1 & 48650.9 & 125 & 235.3 & 8.8 & 85.9 \\
\hline MHT-18C & 102486.1 & 48650.9 & 130 & 230.3 & 692.4 & $1,457.9$ \\
\hline MHT-18C & 102486.1 & 48650.9 & 135 & 225.3 & 436.5 & 981.8 \\
\hline MHT-18C & 102486.1 & 48650.9 & 142 & 218.3 & 141.2 & 123.5 \\
\hline MHT-18C & 102486.1 & 48650.9 & 146 & 214.3 & 449.8 & 529.0 \\
\hline MHT-18C & 102486.1 & 48650.9 & 150 & 210.3 & 705.9 & $2,009.3$ \\
\hline MHT-18C & 102486.1 & 48650.9 & 155 & 205.3 & 9.5 & 33.2 \\
\hline MHT-18C & 102486.1 & 48650.9 & 160 & 200.3 & 349.5 & 161.5 \\
\hline MHT-18C & 102486.1 & 48650.9 & 165 & 195.3 & 122.1 & 47.9 \\
\hline MHT-18C & 102486.1 & 48650.9 & 170 & 190.3 & $1,083.0$ & $2,727.3$ \\
\hline MHT-18C & 102486.1 & 48650.9 & 175 & 185.3 & 800.5 & 826.1 \\
\hline MHT-18C & 102486.1 & 48650.9 & 180 & 180.3 & 856.9 & $1,401.0$ \\
\hline MHT-18C & 102486.1 & 48650.9 & 185 & 175.3 & 527.2 & $1,576.8$ \\
\hline MHT-18C & 102486.1 & 48650.9 & 190 & 170.3 & 664.9 & $2,176.8$ \\
\hline MHT-18C & 102486.1 & 48650.9 & 195 & 165.3 & 357.4 & $1,224.4$ \\
\hline MHT-18C & 102486.1 & 48650.9 & 199 & 161.3 & 438.6 & 275.2 \\
\hline MHT-19C & 102502.7 & 48699.1 & 5 & 353.9 & - & - \\
\hline MHT-19C & 102502.7 & 48699.1 & 10 & 348.9 & - & - \\
\hline MHT-19C & 102502.7 & 48699.1 & 15 & 343.9 & - & - \\
\hline MHT-19C & 102502.7 & 48699.1 & 20 & 338.9 & 544.6 & 2.1 \\
\hline MHT-19C & 102502.7 & 48699.1 & 25 & 333.9 & - & - \\
\hline MHT-19C & 102502.7 & 48699.1 & 30 & 328.9 & - & 15.6 \\
\hline MHT-19C & 102502.7 & 48699.1 & 35 & 323.9 & 767.1 & 531.0 \\
\hline
\end{tabular}


WSRC-TR-2001-00077

February 2001

\begin{tabular}{|c|c|c|c|c|c|c|}
\hline ID & $\begin{array}{l}\text { SRS } \\
\text { North }\end{array}$ & $\begin{array}{l}\text { SRS } \\
\text { East }\end{array}$ & $\begin{array}{l}\text { Sample } \\
\text { Depth } \\
\mathrm{ft}\end{array}$ & $\begin{array}{c}\text { Sample } \\
\text { Elevation } \\
\mathrm{ft} \text { msl }\end{array}$ & $\begin{array}{c}\text { PCE } \\
(\mu \mathrm{g} / \mathrm{kg})\end{array}$ & $\begin{array}{c}\text { TCE } \\
(\mu \mathrm{g} / \mathrm{kg})\end{array}$ \\
\hline MHT-19C & 102502.7 & 48699.1 & 40 & 318.9 & 682.7 & 416.4 \\
\hline MHT-19C & 102502.7 & 48699.1 & 45 & 313.9 & - & - \\
\hline MHT-19C & 102502.7 & 48699.1 & 50 & 308.9 & - & - \\
\hline MHT-19C & 102502.7 & 48699.1 & 55 & 303.9 & - & - \\
\hline MHT-19C & 102502.7 & 48699.1 & 60 & 298.9 & 35.0 & 51.8 \\
\hline MHT-19C & 102502.7 & 48699.1 & 65 & 293.9 & 385.4 & 270.2 \\
\hline MHT-19C & 102502.7 & 48699.1 & 70 & 288.9 & - & - \\
\hline MHT-19C & 102502.7 & 48699.1 & 75 & 283.9 & - & - \\
\hline MHT-19C & 102502.7 & 48699.1 & 80 & 278.9 & - & - \\
\hline MHT-19C & 102502.7 & 48699.1 & 85 & 273.9 & - & - \\
\hline MHT-19C & 102502.7 & 48699.1 & 90 & 268.9 & 477.2 & 341.4 \\
\hline MHT-19C & 102502.7 & 48699.1 & 95 & 263.9 & 582.2 & $1,007.4$ \\
\hline MHT-19C & 102502.7 & 48699.1 & 100 & 258.9 & - & 6.8 \\
\hline MHT-19C & 102502.7 & 48699.1 & 105 & 253.9 & - & - \\
\hline MHT-19C & 102502.7 & 48699.1 & 110 & 248.9 & - & 58.3 \\
\hline MHT-19C & 102502.7 & 48699.1 & 115 & 243.9 & - & - \\
\hline MHT-19C & 102502.7 & 48699.1 & 120 & 238.9 & - & - \\
\hline MHT-19C & 102502.7 & 48699.1 & 125 & 233.9 & - & 62.9 \\
\hline MHT-19C & 102502.7 & 48699.1 & 130 & 228.9 & 317.2 & 592.5 \\
\hline MHT-19C & 102502.7 & 48699.1 & 135 & 223.9 & 467.6 & 490.3 \\
\hline MHT-19C & 102502.7 & 48699.1 & 140 & 218.9 & 475.8 & $1,037.9$ \\
\hline MHT-19C & 102502.7 & 48699.1 & 145 & 213.9 & - & $1,704.7$ \\
\hline MHT-19C & 102502.7 & 48699.1 & 150 & 208.9 & 59.5 & 242.9 \\
\hline MHT-19C & 102502.7 & 48699.1 & 155 & 203.9 & 18.2 & 11.0 \\
\hline MHT-19C & 102502.7 & 48699.1 & 160 & 198.9 & - & - \\
\hline MHT-19C & 102502.7 & 48699.1 & 165 & 193.9 & - & - \\
\hline MHT-19C & 102502.7 & 48699.1 & 170 & 188.9 & - & 281.2 \\
\hline MHT-19C & 102502.7 & 48699.1 & 175 & 183.9 & - & $1,049.0$ \\
\hline MHT-19C & 102502.7 & 48699.1 & 180 & 178.9 & - & $1,280.4$ \\
\hline MHT-19C & 102502.7 & 48699.1 & 185 & 173.9 & - & $1,142.8$ \\
\hline MHT-19C & 102502.7 & 48699.1 & 190 & 168.9 & - & 540.9 \\
\hline MHT-19C & 102502.7 & 48699.1 & 195 & 163.9 & - & 98.9 \\
\hline MHT-19C & 102502.7 & 48699.1 & 198 & 160.9 & - & 21.6 \\
\hline MHT-20C & 102589.3 & 48710.8 & 5 & 355.3 & - & - \\
\hline MHT-20C & 102589.3 & 48710.8 & 10 & 350.3 & - & - \\
\hline MHT-20C & 102589.3 & 48710.8 & 15 & 345.3 & - & - \\
\hline MHT-20C & 102589.3 & 48710.8 & 20 & 340.3 & 104.3 & 7.7 \\
\hline MHT-20C & 102589.3 & 48710.8 & 25 & 335.3 & 6.4 & 3.4 \\
\hline MHT-20C & 102589.3 & 48710.8 & 30 & 330.3 & 14.4 & 17.9 \\
\hline MHT-20C & 102589.3 & 48710.8 & 35 & 325.3 & 411.7 & 161.4 \\
\hline MHT-20C & 102589.3 & 48710.8 & 40 & 320.3 & 432.1 & 252.8 \\
\hline MHT-20C & 102589.3 & 48710.8 & 45 & 315.3 & - & - \\
\hline MHT-20C & 102589.3 & 48710.8 & 50 & 310.3 & - & 34.2 \\
\hline MHT-20C & 102589.3 & 48710.8 & 55 & 305.3 & - & 1.7 \\
\hline MHT-20C & 102589.3 & 48710.8 & 60 & 300.3 & 236.4 & 232.1 \\
\hline MHT-20C & 102589.3 & 48710.8 & 65 & 295.3 & - & 5.5 \\
\hline
\end{tabular}


WSRC-TR-2001-00077 February 2001

\begin{tabular}{|c|c|c|c|c|c|c|}
\hline ID & $\begin{array}{l}\text { SRS } \\
\text { North }\end{array}$ & $\begin{array}{l}\text { SRS } \\
\text { East }\end{array}$ & $\begin{array}{c}\text { Sample } \\
\text { Depth } \\
\mathrm{ft}\end{array}$ & $\begin{array}{c}\text { Sample } \\
\text { Elevation } \\
\mathrm{ft} \text { msl }\end{array}$ & $\begin{array}{c}\text { PCE } \\
(\mu \mathrm{g} / \mathrm{kg})\end{array}$ & $\begin{array}{c}\text { TCE } \\
(\mu \mathrm{g} / \mathrm{kg})\end{array}$ \\
\hline MHT-20C & 102589.3 & 48710.8 & 70 & 290.3 & - & - \\
\hline MHT-20C & 102589.3 & 48710.8 & 75 & 285.3 & - & - \\
\hline MHT-20C & 102589.3 & 48710.8 & 80 & 280.3 & - & - \\
\hline MHT-20C & 102589.3 & 48710.8 & 85 & 275.3 & 280.3 & 209.0 \\
\hline MHT-20C & 102589.3 & 48710.8 & 90 & 270.3 & 342.8 & 276.8 \\
\hline MHT-20C & 102589.3 & 48710.8 & 95 & 265.3 & - & 18.8 \\
\hline MHT-20C & 102589.3 & 48710.8 & 100 & 260.3 & - & - \\
\hline MHT-20C & 102589.3 & 48710.8 & 105 & 255.3 & - & - \\
\hline MHT-20C & 102589.3 & 48710.8 & 110 & 250.3 & - & - \\
\hline MHT-20C & 102589.3 & 48710.8 & 115 & 245.3 & - & - \\
\hline MHT-20C & 102589.3 & 48710.8 & 120 & 240.3 & - & - \\
\hline MHT-20C & 102589.3 & 48710.8 & 125 & 235.3 & - & - \\
\hline MHT-20C & 102589.3 & 48710.8 & 130 & 230.3 & - & - \\
\hline MHT-20C & 102589.3 & 48710.8 & 135 & 225.3 & - & - \\
\hline MHT-20C & 102589.3 & 48710.8 & 140 & 220.3 & 14.9 & 53.2 \\
\hline MHT-20C & 102589.3 & 48710.8 & 145 & 215.3 & - & - \\
\hline MHT-20C & 102589.3 & 48710.8 & 150 & 210.3 & - & - \\
\hline MHT-20C & 102589.3 & 48710.8 & 155 & 205.3 & 359.7 & 546.4 \\
\hline MHT-20C & 102589.3 & 48710.8 & 160 & 200.3 & 101.3 & 47.1 \\
\hline MHT-20C & 102589.3 & 48710.8 & 165 & 195.3 & - & - \\
\hline MHT-20C & 102589.3 & 48710.8 & 170 & 190.3 & - & 4.1 \\
\hline MHT-20C & 102589.3 & 48710.8 & 175 & 185.3 & - & 10.8 \\
\hline MHT-20C & 102589.3 & 48710.8 & 180 & 180.3 & - & 885.9 \\
\hline MHT-20C & 102589.3 & 48710.8 & 185 & 175.3 & - & $1,499.0$ \\
\hline MHT-20C & 102589.3 & 48710.8 & 190 & 170.3 & - & 551.2 \\
\hline MHT-2C & 102747.1 & 48780.28 & 5 & 359.1 & - & - \\
\hline MHV-1 & 102749.3 & 48841.98 & 1 & 364.3 & 261.0 & $1,595.0$ \\
\hline MHV-1 & 102749.3 & 48841.98 & 19 & 346.3 & 28.0 & 15.0 \\
\hline MHV-1 & 102749.3 & 48841.98 & 20 & 345.3 & - & - \\
\hline MHV-1 & 102749.3 & 48841.98 & 21 & 344.3 & 94.0 & 45.0 \\
\hline MHV-1 & 102749.3 & 48841.98 & 22 & 343.3 & 173.0 & 79.0 \\
\hline MHV-1 & 102749.3 & 48841.98 & 22.5 & 342.8 & 227.0 & 102.0 \\
\hline MHV-1 & 102749.3 & 48841.98 & 24 & 341.3 & 355.0 & 156.0 \\
\hline MHV-1 & 102749.3 & 48841.98 & 26 & 339.3 & 218.0 & 97.0 \\
\hline MHV-1 & 102749.3 & 48841.98 & 28 & 337.3 & 29.0 & 25.0 \\
\hline MHV-1 & 102749.3 & 48841.98 & 29 & 336.3 & 33.0 & 22.0 \\
\hline MHV-1 & 102749.3 & 48841.98 & 30 & 335.3 & 14.0 & 12.0 \\
\hline MHV-1 & 102749.3 & 48841.98 & 31 & 334.3 & - & - \\
\hline MHV-1 & 102749.3 & 48841.98 & 32 & 333.3 & 17.0 & 12.0 \\
\hline MHV-1 & 102749.3 & 48841.98 & 33 & 332.3 & 430.0 & 213.0 \\
\hline MHV-1 & 102749.3 & 48841.98 & 34 & 331.3 & 13.0 & 11.0 \\
\hline MHV-1 & 102749.3 & 48841.98 & 35 & 330.3 & 89.0 & 54.0 \\
\hline MHV-1 & 102749.3 & 48841.98 & 36 & 329.3 & 36.0 & 27.0 \\
\hline MHV-1 & 102749.3 & 48841.98 & 37 & 328.3 & 155.0 & 83.0 \\
\hline MHV-1 & 102749.3 & 48841.98 & 38 & 327.3 & - & - \\
\hline MHV-1 & 102749.3 & 48841.98 & 39 & 326.3 & 508.0 & 227.0 \\
\hline
\end{tabular}


WSRC-TR-2001-00077 February 2001

\begin{tabular}{|c|c|c|c|c|c|c|}
\hline ID & $\begin{array}{c}\text { SRS } \\
\text { North } \\
\end{array}$ & $\begin{array}{l}\text { SRS } \\
\text { East }\end{array}$ & $\begin{array}{c}\text { Sample } \\
\text { Depth } \\
\mathrm{ft}\end{array}$ & $\begin{array}{c}\text { Sample } \\
\text { Elevation } \\
\mathrm{ft} \mathrm{msl}\end{array}$ & $\begin{array}{c}\text { PCE } \\
(\mu \mathrm{g} / \mathrm{kg})\end{array}$ & $\begin{array}{c}\text { TCE } \\
(\mu \mathrm{g} / \mathrm{kg})\end{array}$ \\
\hline MHV-1 & 102749.3 & 48841.98 & 40 & 325.3 & - & - \\
\hline MHV-1 & 102749.3 & 48841.98 & 41 & 324.3 & 41.0 & 31.0 \\
\hline MHV-1 & 102749.3 & 48841.98 & 42 & 323.3 & $1,440.0$ & $1,473.0$ \\
\hline MHV-1 & 102749.3 & 48841.98 & 43 & 322.3 & 75.0 & 63.0 \\
\hline MHV-1 & 102749.3 & 48841.98 & 44 & 321.3 & 58.0 & 70.0 \\
\hline MHV-1 & 102749.3 & 48841.98 & 45 & 320.3 & - & - \\
\hline MHV-1 & 102749.3 & 48841.98 & 46 & 319.3 & 596.0 & 328.0 \\
\hline MHV-1 & 102749.3 & 48841.98 & 47 & 318.3 & 26.0 & 23.0 \\
\hline MHV-1 & 102749.3 & 48841.98 & 48 & 317.3 & 399.0 & 223.0 \\
\hline $\mathrm{MHV}-1$ & 102749.3 & 48841.98 & 49 & 316.3 & $1,201.0$ & 944.0 \\
\hline MHV-1 & 102749.3 & 48841.98 & 50 & 315.3 & 425.0 & 236.0 \\
\hline MHV-1 & 102749.3 & 48841.98 & 51 & 314.3 & 14.0 & 4.0 \\
\hline MHV-1 & 102749.3 & 48841.98 & 52 & 313.3 & 20.0 & 18.0 \\
\hline MHV-1 & 102749.3 & 48841.98 & 53 & 312.3 & 13.0 & 11.0 \\
\hline MHV-1 & 102749.3 & 48841.98 & 54 & 311.3 & 416.0 & 195.0 \\
\hline MHV-1 & 102749.3 & 48841.98 & 55 & 310.3 & 37.0 & 35.0 \\
\hline MHV-1 & 102749.3 & 48841.98 & 56 & 309.3 & 345.0 & 152.0 \\
\hline $\mathrm{MHV}-1$ & 102749.3 & 48841.98 & 57 & 308.3 & 9.0 & 6.0 \\
\hline MHV-1 & 102749.3 & 48841.98 & 58 & 307.3 & 143.0 & 76.0 \\
\hline MHV-1 & 102749.3 & 48841.98 & 59 & 306.3 & 546.0 & 591.0 \\
\hline MHV-1 & 102749.3 & 48841.98 & 60 & 305.3 & 643.0 & 428.0 \\
\hline MHV-1 & 102749.3 & 48841.98 & 62 & 303.3 & 12.0 & 21.0 \\
\hline MHV-1 & 102749.3 & 48841.98 & 63 & 302.3 & 822.0 & $1,073.0$ \\
\hline MHV-1 & 102749.3 & 48841.98 & 64 & 301.3 & - & - \\
\hline MHV-1 & 102749.3 & 48841.98 & 65 & 300.3 & $1,511.0$ & $2,006.0$ \\
\hline MHV-1 & 102749.3 & 48841.98 & 66 & 299.3 & $1,606.0$ & $1,739.0$ \\
\hline MHV-1 & 102749.3 & 48841.98 & 67 & 298.3 & 901.0 & $1,335.0$ \\
\hline MHV-1 & 102749.3 & 48841.98 & 68 & 297.3 & 755.0 & $1,191.0$ \\
\hline MHV-1 & 102749.3 & 48841.98 & 69 & 296.3 & 6.0 & 7.0 \\
\hline MHV-1 & 102749.3 & 48841.98 & 70 & 295.3 & 27.0 & 44.0 \\
\hline MHV-1 & 102749.3 & 48841.98 & 71 & 294.3 & 221.0 & 263.0 \\
\hline MHV-1 & 102749.3 & 48841.98 & 72 & 293.3 & - & - \\
\hline MHV-1 & 102749.3 & 48841.98 & 73 & 292.3 & - & - \\
\hline MHV-1 & 102749.3 & 48841.98 & 74 & 291.3 & - & - \\
\hline MHV-1 & 102749.3 & 48841.98 & 75 & 290.3 & - & - \\
\hline MHV-1 & 102749.3 & 48841.98 & 76 & 289.3 & 47.0 & 56.0 \\
\hline MHV-1 & 102749.3 & 48841.98 & 77 & 288.3 & - & - \\
\hline MHV-1 & 102749.3 & 48841.98 & 78 & 287.3 & - & - \\
\hline MHV-1 & 102749.3 & 48841.98 & 79 & 286.3 & - & - \\
\hline MHV-1 & 102749.3 & 48841.98 & 80 & 285.3 & - & - \\
\hline MHV-1 & 102749.3 & 48841.98 & 81 & 284.3 & - & - \\
\hline MHV-1 & 102749.3 & 48841.98 & 82 & 283.3 & - & - \\
\hline MHV-1 & 102749.3 & 48841.98 & 83 & 282.3 & - & - \\
\hline MHV-1 & 102749.3 & 48841.98 & 84 & 281.3 & - & - \\
\hline MHV-1 & 102749.3 & 48841.98 & 85 & 280.3 & - & - \\
\hline MHV-1 & 102749.3 & 48841.98 & 86 & 279.3 & - & - \\
\hline
\end{tabular}


WSRC-TR-2001-00077 February 2001

\begin{tabular}{|c|c|c|c|c|c|c|}
\hline ID & $\begin{array}{c}\text { SRS } \\
\text { North } \\
\end{array}$ & $\begin{array}{l}\text { SRS } \\
\text { East }\end{array}$ & $\begin{array}{c}\text { Sample } \\
\text { Depth } \\
\mathrm{ft}\end{array}$ & $\begin{array}{c}\text { Sample } \\
\text { Elevation } \\
\mathrm{ft} \mathrm{msl}\end{array}$ & $\begin{array}{c}\text { PCE } \\
(\mu \mathrm{g} / \mathrm{kg})\end{array}$ & $\begin{array}{c}\text { TCE } \\
(\mu \mathrm{g} / \mathrm{kg})\end{array}$ \\
\hline MHV-1 & 102749.3 & 48841.98 & 87 & 278.3 & - & - \\
\hline MHV-1 & 102749.3 & 48841.98 & 88 & 277.3 & - & - \\
\hline MHV-1 & 102749.3 & 48841.98 & 89 & 276.3 & - & - \\
\hline MHV-1 & 102749.3 & 48841.98 & 90 & 275.3 & - & - \\
\hline MHV-1 & 102749.3 & 48841.98 & 91 & 274.3 & - & - \\
\hline MHV-1 & 102749.3 & 48841.98 & 92 & 273.3 & - & - \\
\hline MHV-1 & 102749.3 & 48841.98 & 93 & 272.3 & - & - \\
\hline MHV-1 & 102749.3 & 48841.98 & 94 & 271.3 & $4,925.0$ & $11,026.0$ \\
\hline MHV-1 & 102749.3 & 48841.98 & 94.5 & 270.8 & 975.0 & $1,674.0$ \\
\hline MHV-1 & 102749.3 & 48841.98 & 95 & 270.3 & 829.0 & $1,744.0$ \\
\hline MHV-1 & 102749.3 & 48841.98 & 96 & 269.3 & 334.0 & 646.0 \\
\hline MHV-1 & 102749.3 & 48841.98 & 97 & 268.3 & 95.0 & 311.0 \\
\hline MHV-1 & 102749.3 & 48841.98 & 98 & 267.3 & - & - \\
\hline MHV-1 & 102749.3 & 48841.98 & 99 & 266.3 & - & - \\
\hline MHV-1 & 102749.3 & 48841.98 & 100 & 265.3 & - & - \\
\hline MHV-1 & 102749.3 & 48841.98 & 101 & 264.3 & - & - \\
\hline MHV-1 & 102749.3 & 48841.98 & 102 & 263.3 & - & - \\
\hline $\mathrm{MHV}-1$ & 102749.3 & 48841.98 & 103 & 262.3 & 656.0 & $5,138.0$ \\
\hline MHV-1 & 102749.3 & 48841.98 & 104 & 261.3 & $1,357.0$ & $6,282.0$ \\
\hline MHV-1 & 102749.3 & 48841.98 & 105 & 260.3 & 824.0 & $6,701.0$ \\
\hline MHV-1 & 102749.3 & 48841.98 & 106 & 259.3 & $4,161.0$ & $14,458.0$ \\
\hline MHV-1 & 102749.3 & 48841.98 & 107 & 258.3 & 611.0 & $8,058.0$ \\
\hline MHV-1 & 102749.3 & 48841.98 & 108 & 257.3 & 390.0 & $7,672.0$ \\
\hline MHV-1 & 102749.3 & 48841.98 & 109 & 256.3 & 878.0 & $7,450.0$ \\
\hline MHV-1 & 102749.3 & 48841.98 & 110 & 255.3 & 776.0 & $3,508.0$ \\
\hline MHV-1 & 102749.3 & 48841.98 & 112 & 253.3 & $2,950.0$ & $7,022.0$ \\
\hline MHV-1 & 102749.3 & 48841.98 & 114 & 251.3 & $1,710.0$ & $6,903.0$ \\
\hline MHV-1 & 102749.3 & 48841.98 & 115 & 250.3 & $1,554.0$ & $5,870.0$ \\
\hline MHV-1 & 102749.3 & 48841.98 & 116 & 249.3 & 292.0 & $1,128.0$ \\
\hline MHV-1 & 102749.3 & 48841.98 & 117 & 248.3 & 37.0 & $1,636.0$ \\
\hline MHV-1 & 102749.3 & 48841.98 & 118 & 247.3 & - & 172.0 \\
\hline MHV-1 & 102749.3 & 48841.98 & 119 & 246.3 & 166.0 & $1,597.0$ \\
\hline MHV-1 & 102749.3 & 48841.98 & 120 & 245.3 & 4.0 & 114.0 \\
\hline MHV-1 & 102749.3 & 48841.98 & 121 & 244.3 & 29.0 & 358.0 \\
\hline MHV-1 & 102749.3 & 48841.98 & 122 & 243.3 & - & 9.0 \\
\hline MHV-1 & 102749.3 & 48841.98 & 123 & 242.3 & 108.0 & $1,456.0$ \\
\hline MHV-1 & 102749.3 & 48841.98 & 124 & 241.3 & - & 28.0 \\
\hline MHV-1 & 102749.3 & 48841.98 & 125 & 240.3 & 15.0 & 441.0 \\
\hline MHV-1 & 102749.3 & 48841.98 & 126 & 239.3 & 32.0 & 695.0 \\
\hline MHV-1 & 102749.3 & 48841.98 & 127 & 238.3 & 100.0 & 959.0 \\
\hline MHV-1 & 102749.3 & 48841.98 & 128 & 237.3 & 83.0 & 665.0 \\
\hline MHV-1 & 102749.3 & 48841.98 & 129 & 236.3 & 103.0 & 780.0 \\
\hline MHV-1 & 102749.3 & 48841.98 & 130 & 235.3 & - & - \\
\hline MHV-2 & 102755.9 & 48903.22 & 15 & 351 & - & - \\
\hline MHV-2 & 102755.9 & 48903.22 & 20 & 346 & - & - \\
\hline MHV-2 & 102755.9 & 48903.22 & 25 & 341 & - & - \\
\hline
\end{tabular}


WSRC-TR-2001-00077 February 2001

\begin{tabular}{|c|c|c|c|c|c|c|}
\hline ID & $\begin{array}{c}\text { SRS } \\
\text { North } \\
\end{array}$ & $\begin{array}{l}\text { SRS } \\
\text { East }\end{array}$ & $\begin{array}{c}\text { Sample } \\
\text { Depth } \\
\mathrm{ft}\end{array}$ & $\begin{array}{c}\text { Sample } \\
\text { Elevation } \\
\mathrm{ft} \mathrm{msl}\end{array}$ & $\begin{array}{c}\text { PCE } \\
(\mu \mathrm{g} / \mathrm{kg})\end{array}$ & $\begin{array}{c}\text { TCE } \\
(\mu \mathrm{g} / \mathrm{kg})\end{array}$ \\
\hline MHV-2 & 102755.9 & 48903.22 & 30 & 336 & - & - \\
\hline MHV-2 & 102755.9 & 48903.22 & 35 & 331 & - & - \\
\hline MHV-2 & 102755.9 & 48903.22 & 40 & 326 & 46.0 & 42.0 \\
\hline MHV-2 & 102755.9 & 48903.22 & 41 & 325 & - & - \\
\hline MHV-2 & 102755.9 & 48903.22 & 46 & 320 & 35.6 & 27.6 \\
\hline MHV-2 & 102755.9 & 48903.22 & 50 & 316 & 69.0 & 50.0 \\
\hline MHV-2 & 102755.9 & 48903.22 & 51 & 315 & 235.0 & 180.7 \\
\hline MHV-2 & 102755.9 & 48903.22 & 55 & 311 & 33.0 & 42.0 \\
\hline MHV-2 & 102755.9 & 48903.22 & 60 & 306 & 95.0 & 77.3 \\
\hline MHV-2 & 102755.9 & 48903.22 & 64 & 302 & 151.0 & 139.7 \\
\hline MHV-2 & 102755.9 & 48903.22 & 69 & 297 & 19.3 & 44.7 \\
\hline MHV-2 & 102755.9 & 48903.22 & 75 & 291 & - & - \\
\hline MHV-2 & 102755.9 & 48903.22 & 79 & 287 & - & - \\
\hline MHV-2 & 102755.9 & 48903.22 & 85 & 281 & - & - \\
\hline MHV-2 & 102755.9 & 48903.22 & 90 & 276 & - & - \\
\hline MHV-2 & 102755.9 & 48903.22 & 95 & 271 & $1,125.5$ & $5,244.2$ \\
\hline MHV-2 & 102755.9 & 48903.22 & 100 & 266 & 30.7 & 146.3 \\
\hline MHV-2 & 102755.9 & 48903.22 & 105 & 261 & 625.7 & $2,107.3$ \\
\hline MHV-2 & 102755.9 & 48903.22 & 108 & 258 & 662.0 & $4,989.0$ \\
\hline MHV-2 & 102755.9 & 48903.22 & 110 & 256 & $1,725.0$ & $4,223.5$ \\
\hline MHV-2 & 102755.9 & 48903.22 & 112 & 254 & 103.5 & $2,909.5$ \\
\hline MHV-2 & 102755.9 & 48903.22 & 115 & 251 & 528.5 & $1,694.0$ \\
\hline MHV-2 & 102755.9 & 48903.22 & 120 & 246 & 83.0 & $1,359.0$ \\
\hline MHV-3 & 102774.7 & 48874.06 & 5 & 362.9 & - & - \\
\hline MHV-3 & 102774.7 & 48874.06 & 15 & 352.9 & - & - \\
\hline MHV-3 & 102774.7 & 48874.06 & 25 & 342.9 & - & - \\
\hline MHV-3 & 102774.7 & 48874.06 & 33 & 334.9 & - & - \\
\hline MHV-3 & 102774.7 & 48874.06 & 39 & 328.9 & 71.0 & 35.0 \\
\hline MHV-3 & 102774.7 & 48874.06 & 45 & 322.9 & 169.0 & 56.0 \\
\hline MHV-3 & 102774.7 & 48874.06 & 55 & 312.9 & 109.0 & 77.0 \\
\hline MHV-3 & 102774.7 & 48874.06 & 65 & 302.9 & 398.0 & 228.0 \\
\hline MHV-3 & 102774.7 & 48874.06 & 67 & 300.9 & 486.7 & 387.3 \\
\hline MHV-3 & 102774.7 & 48874.06 & 75 & 292.9 & 641.0 & $1,281.0$ \\
\hline MHV-4 & 102841.7 & 48842.53 & 20 & 345.7 & - & - \\
\hline MHV-4 & 102841.7 & 48842.53 & 25 & 340.7 & 224.3 & 1.3 \\
\hline MHV-4 & 102841.7 & 48842.53 & 30 & 335.7 & 144.0 & 25.7 \\
\hline MHV-4 & 102841.7 & 48842.53 & 35 & 330.7 & 557.0 & 489.0 \\
\hline MHV-4 & 102841.7 & 48842.53 & 40 & 325.7 & 65.5 & 46.0 \\
\hline MHV-4 & 102841.7 & 48842.53 & 43 & 322.7 & $6,828.3$ & $7,300.0$ \\
\hline MHV-4 & 102841.7 & 48842.53 & 45 & 320.7 & $6,915.3$ & $6,157.7$ \\
\hline MHV-4 & 102841.7 & 48842.53 & 50 & 315.7 & 18.7 & 29.3 \\
\hline MHV-4 & 102841.7 & 48842.53 & 55 & 310.7 & 434.3 & 647.7 \\
\hline MHV-4 & 102841.7 & 48842.53 & 60 & 305.7 & 118.3 & 154.3 \\
\hline MHV-4 & 102841.7 & 48842.53 & 65 & 300.7 & $3,425.3$ & $6,646.0$ \\
\hline MHV-4 & 102841.7 & 48842.53 & 70 & 295.7 & 268.0 & 361.7 \\
\hline MHV-4 & 102841.7 & 48842.53 & 75 & 290.7 & 34.3 & 183.3 \\
\hline
\end{tabular}


WSRC-TR-2001-00077 February 2001

\begin{tabular}{|c|c|c|c|c|c|c|}
\hline ID & $\begin{array}{c}\text { SRS } \\
\text { North }\end{array}$ & $\begin{array}{l}\text { SRS } \\
\text { East }\end{array}$ & $\begin{array}{c}\text { Sample } \\
\text { Depth } \\
\mathrm{ft}\end{array}$ & $\begin{array}{c}\text { Sample } \\
\text { Elevation } \\
\mathrm{ft} \text { msl }\end{array}$ & $\begin{array}{c}\text { PCE } \\
(\mu \mathrm{g} / \mathrm{kg})\end{array}$ & $\begin{array}{c}\text { TCE } \\
(\mu \mathrm{g} / \mathrm{kg})\end{array}$ \\
\hline MHV-4 & 102841.7 & 48842.53 & 83 & 282.7 & 93.7 & 350.7 \\
\hline MHV-4 & 102841.7 & 48842.53 & 85 & 280.7 & 23.0 & 101.3 \\
\hline MHV-4 & 102841.7 & 48842.53 & 90 & 275.7 & - & - \\
\hline MHV-4 & 102841.7 & 48842.53 & 94 & 271.7 & $2,381.0$ & $9,241.7$ \\
\hline MHV-4 & 102841.7 & 48842.53 & 95 & 270.7 & 644.3 & $5,779.0$ \\
\hline MHV-4 & 102841.7 & 48842.53 & 100 & 265.7 & 246.3 & $3,346.0$ \\
\hline MHV-4 & 102841.7 & 48842.53 & 105 & 260.7 & $1,053.3$ & $13,314.0$ \\
\hline MHV-4 & 102841.7 & 48842.53 & 107 & 258.7 & 265.3 & $8,329.0$ \\
\hline MHV-4 & 102841.7 & 48842.53 & 110 & 255.7 & 60.7 & $1,731.7$ \\
\hline MHV-4 & 102841.7 & 48842.53 & 115 & 250.7 & 445.7 & $4,280.7$ \\
\hline MHV-4 & 102841.7 & 48842.53 & 120 & 245.7 & - & - \\
\hline MHV-4 & 102841.7 & 48842.53 & 125 & 240.7 & - & - \\
\hline MHV-4 & 102841.7 & 48842.53 & 130 & 235.7 & 5.7 & 80.0 \\
\hline MHV-5 & 102878.8 & 48917.46 & 15 & 353.9 & - & - \\
\hline MHV-5 & 102878.8 & 48917.46 & 21 & 347.9 & - & - \\
\hline MHV-5 & 102878.8 & 48917.46 & 25 & 343.9 & - & - \\
\hline MHV-5 & 102878.8 & 48917.46 & 27 & 341.9 & - & - \\
\hline MHV-5 & 102878.8 & 48917.46 & 31 & 337.9 & - & - \\
\hline MHV-5 & 102878.8 & 48917.46 & 35 & 333.9 & - & - \\
\hline MHV-5 & 102878.8 & 48917.46 & 40 & 328.9 & 4.7 & 14.7 \\
\hline MHV-5 & 102878.8 & 48917.46 & 45 & 323.9 & 2.7 & 7.3 \\
\hline MHV-5 & 102878.8 & 48917.46 & 50 & 318.9 & 88.0 & 160.0 \\
\hline MHV-5 & 102878.8 & 48917.46 & 55 & 313.9 & 229.7 & 638.7 \\
\hline MHV-5 & 102878.8 & 48917.46 & 60 & 308.9 & 93.3 & 470.7 \\
\hline MHV-5 & 102878.8 & 48917.46 & 65 & 303.9 & 114.3 & 766.0 \\
\hline MHV-5 & 102878.8 & 48917.46 & 70 & 298.9 & 103.0 & 689.7 \\
\hline MHV-5 & 102878.8 & 48917.46 & 75 & 293.9 & 215.0 & $1,503.0$ \\
\hline MHV-5 & 102878.8 & 48917.46 & 80 & 288.9 & 635.0 & $5,948.3$ \\
\hline MHV-5 & 102878.8 & 48917.46 & 85 & 283.9 & 9.7 & 125.3 \\
\hline MHV-5 & 102878.8 & 48917.46 & 90 & 278.9 & 32.3 & 488.0 \\
\hline MHV-5 & 102878.8 & 48917.46 & 91 & 277.9 & 1.3 & 44.0 \\
\hline MHV-5 & 102878.8 & 48917.46 & 95 & 273.9 & - & 25.0 \\
\hline MHV-5 & 102878.8 & 48917.46 & 100 & 268.9 & 575.3 & $1,964.3$ \\
\hline MHV-5 & 102878.8 & 48917.46 & 105 & 263.9 & 401.3 & $1,393.0$ \\
\hline MHV-5 & 102878.8 & 48917.46 & 110 & 258.9 & 387.3 & $2,632.7$ \\
\hline MHV-5 & 102878.8 & 48917.46 & 115 & 253.9 & 538.0 & $4,437.3$ \\
\hline MHV-5 & 102878.8 & 48917.46 & 117 & 251.9 & 520.3 & $7,096.3$ \\
\hline MHV-5 & 102878.8 & 48917.46 & 120 & 248.9 & 345.3 & $7,044.0$ \\
\hline MHV-5 & 102878.8 & 48917.46 & 125 & 243.9 & 88.0 & $1,405.3$ \\
\hline MHV-5 & 102878.8 & 48917.46 & 130 & 238.9 & 370.0 & $13,072.7$ \\
\hline MHV-20 & 102418.4 & 48658.3 & 24.9 & 334.7 & 9.0 & 8.0 \\
\hline MHV-20 & 102418.4 & 48658.3 & 26.9 & 332.7 & 140.0 & 50.0 \\
\hline MHV-20 & 102418.4 & 48658.3 & 28.9 & 330.7 & 2.0 & 6.0 \\
\hline MHV-20 & 102418.4 & 48658.3 & 30.9 & 328.7 & 9.0 & - \\
\hline MHV-20 & 102418.4 & 48658.3 & 32.9 & 326.7 & 4.0 & 1.0 \\
\hline MHV-20 & 102418.4 & 48658.3 & 34.9 & 324.7 & 4.0 & 6.0 \\
\hline
\end{tabular}


WSRC-TR-2001-00077

February 2001

\begin{tabular}{|c|c|c|c|c|c|c|}
\hline ID & $\begin{array}{l}\text { SRS } \\
\text { North }\end{array}$ & $\begin{array}{l}\text { SRS } \\
\text { East }\end{array}$ & $\begin{array}{l}\text { Sample } \\
\text { Depth } \\
\mathrm{ft}\end{array}$ & $\begin{array}{c}\text { Sample } \\
\text { Elevation } \\
\mathrm{ft} \text { msl }\end{array}$ & $\begin{array}{c}\text { PCE } \\
(\mu \mathrm{g} / \mathrm{kg})\end{array}$ & $\begin{array}{c}\text { TCE } \\
(\mu \mathrm{g} / \mathrm{kg})\end{array}$ \\
\hline MHV-20 & 102418.4 & 48658.3 & 35.9 & 323.7 & 140.0 & 100.0 \\
\hline MHV-20 & 102418.4 & 48658.3 & 36.9 & 322.7 & 390.0 & 130.0 \\
\hline MHV-20 & 102418.4 & 48658.3 & 37.9 & 321.7 & $1,020.0$ & 540.0 \\
\hline MHV-20 & 102418.4 & 48658.3 & 38.9 & 320.7 & $1,620.0$ & $2,390.0$ \\
\hline MHV-20 & 102418.4 & 48658.3 & 39.9 & 319.7 & $1,120.0$ & $1,760.0$ \\
\hline MHV-20 & 102418.4 & 48658.3 & 40.9 & 318.7 & $2,070.0$ & $3,720.0$ \\
\hline MHV-20 & 102418.4 & 48658.3 & 41.9 & 317.7 & 650.0 & $1,700.0$ \\
\hline MHV-20 & 102418.4 & 48658.3 & 42.9 & 316.7 & 810.0 & $1,940.0$ \\
\hline MHV-20 & 102418.4 & 48658.3 & 43.9 & 315.7 & $2,320.0$ & $7,690.0$ \\
\hline MHV-20 & 102418.4 & 48658.3 & 44.9 & 314.7 & $1,010.0$ & $2,510.0$ \\
\hline MHV-20 & 102418.4 & 48658.3 & 46.9 & 312.7 & 10.0 & 10.0 \\
\hline MHV-20 & 102418.4 & 48658.3 & 48.9 & 310.7 & 10.0 & 30.0 \\
\hline MHV-20 & 102418.4 & 48658.3 & 50.9 & 308.7 & 6.0 & 7.0 \\
\hline MHV-20 & 102418.4 & 48658.3 & 52.9 & 306.7 & 6.0 & 8.0 \\
\hline MHV-20 & 102418.4 & 48658.3 & 54.9 & 304.7 & 20.0 & 20.0 \\
\hline MHV-20 & 102418.4 & 48658.3 & 56.9 & 302.7 & 20.0 & 30.0 \\
\hline MHV-20 & 102418.4 & 48658.3 & 58.9 & 300.7 & 20.0 & 30.0 \\
\hline MHV-21 & 102433.6 & 48661.47 & 25 & 334.7 & 9.0 & 6.0 \\
\hline MHV-21 & 102433.6 & 48661.47 & 27 & 332.7 & 20.0 & 20.0 \\
\hline MHV-21 & 102433.6 & 48661.47 & 29 & 330.7 & 10.0 & 10.0 \\
\hline MHV-21 & 102433.6 & 48661.47 & 31 & 328.7 & 10.0 & 6.0 \\
\hline MHV-21 & 102433.6 & 48661.47 & 33 & 326.7 & 40.0 & 10.0 \\
\hline MHV-21 & 102433.6 & 48661.47 & 35 & 324.7 & $1,370.0$ & 600.0 \\
\hline MHV-21 & 102433.6 & 48661.47 & 36 & 323.7 & 870.0 & 330.0 \\
\hline MHV-21 & 102433.6 & 48661.47 & 37 & 322.7 & 40.0 & 10.0 \\
\hline MHV-21 & 102433.6 & 48661.47 & 38 & 321.7 & 710.0 & 950.0 \\
\hline MHV-21 & 102433.6 & 48661.47 & 39 & 320.7 & 160.0 & 460.0 \\
\hline MHV-21 & 102433.6 & 48661.47 & 40 & 319.7 & 970.0 & $4,860.0$ \\
\hline MHV-21 & 102433.6 & 48661.47 & 41 & 318.7 & $1,490.0$ & $9,400.0$ \\
\hline MHV-21 & 102433.6 & 48661.47 & 42 & 317.7 & 50.0 & 110.0 \\
\hline MHV-21 & 102433.6 & 48661.47 & 43 & 316.7 & 80.0 & 120.0 \\
\hline MHV-21 & 102433.6 & 48661.47 & 44 & 315.7 & 70.0 & 100.0 \\
\hline MHV-21 & 102433.6 & 48661.47 & 45 & 314.7 & 10.0 & 10.0 \\
\hline MHV-21 & 102433.6 & 48661.47 & 47 & 312.7 & 10.0 & 2.0 \\
\hline MHV-21 & 102433.6 & 48661.47 & 49 & 310.7 & 10.0 & 6.0 \\
\hline MHV-21 & 102433.6 & 48661.47 & 51 & 308.7 & 7.0 & 1.0 \\
\hline MHV-21 & 102433.6 & 48661.47 & 53 & 306.7 & 10.0 & - \\
\hline MHV-21 & 102433.6 & 48661.47 & 55 & 304.7 & 10.0 & 2.0 \\
\hline MHV-21 & 102433.6 & 48661.47 & 57 & 302.7 & 4.0 & - \\
\hline MHV-21 & 102433.6 & 48661.47 & 59 & 300.7 & 40.0 & 50.0 \\
\hline MHV-22 & 102443.5 & 48663.79 & 25 & 334.7 & - & - \\
\hline MHV-22 & 102443.5 & 48663.79 & 27 & 332.7 & - & - \\
\hline MHV-22 & 102443.5 & 48663.79 & 29 & 330.7 & - & - \\
\hline MHV-22 & 102443.5 & 48663.79 & 31 & 328.7 & - & - \\
\hline MHV-22 & 102443.5 & 48663.79 & 33 & 326.7 & - & - \\
\hline MHV-22 & 102443.5 & 48663.79 & 35 & 324.7 & 170.0 & 240.0 \\
\hline
\end{tabular}


WSRC-TR-2001-00077

February 2001

\begin{tabular}{|c|c|c|c|c|c|c|}
\hline ID & $\begin{array}{l}\text { SRS } \\
\text { North }\end{array}$ & $\begin{array}{l}\text { SRS } \\
\text { East }\end{array}$ & $\begin{array}{l}\text { Sample } \\
\text { Depth } \\
\mathrm{ft}\end{array}$ & $\begin{array}{c}\text { Sample } \\
\text { Elevation } \\
\mathrm{ft} \text { msl }\end{array}$ & $\begin{array}{c}\text { PCE } \\
(\mu \mathrm{g} / \mathrm{kg})\end{array}$ & $\begin{array}{c}\text { TCE } \\
(\mu \mathrm{g} / \mathrm{kg})\end{array}$ \\
\hline MHV-22 & 102443.5 & 48663.79 & 36 & 323.7 & 20.0 & 30.0 \\
\hline MHV-22 & 102443.5 & 48663.79 & 37 & 322.7 & 5.0 & 5.0 \\
\hline MHV-22 & 102443.5 & 48663.79 & 38 & 321.7 & 2.0 & 1.0 \\
\hline MHV-22 & 102443.5 & 48663.79 & 39 & 320.7 & 10.0 & 10.0 \\
\hline MHV-22 & 102443.5 & 48663.79 & 40 & 319.7 & 50.0 & 30.0 \\
\hline MHV-22 & 102443.5 & 48663.79 & 41 & 318.7 & 3.0 & 2.0 \\
\hline MHV-22 & 102443.5 & 48663.79 & 42 & 317.7 & - & - \\
\hline MHV-22 & 102443.5 & 48663.79 & 43 & 316.7 & - & - \\
\hline MHV-22 & 102443.5 & 48663.79 & 44 & 315.7 & - & - \\
\hline MHV-22 & 102443.5 & 48663.79 & 45 & 314.7 & 40.0 & 50.0 \\
\hline MHV-22 & 102443.5 & 48663.79 & 47 & 312.7 & - & - \\
\hline MHV-22 & 102443.5 & 48663.79 & 49 & 310.7 & - & - \\
\hline MHV-22 & 102443.5 & 48663.79 & 51 & 308.7 & - & - \\
\hline MHV-22 & 102443.5 & 48663.79 & 53 & 306.7 & - & - \\
\hline MHV-22 & 102443.5 & 48663.79 & 55 & 304.7 & 10.0 & 10.0 \\
\hline MHV-22 & 102443.5 & 48663.79 & 57 & 302.7 & 6.0 & 7.0 \\
\hline MHV-22 & 102443.5 & 48663.79 & 59 & 300.7 & 100.0 & 80.0 \\
\hline MHV-23 & 102458 & 48666.89 & 24.9 & 334.8 & 20.0 & 6.0 \\
\hline MHV-23 & 102458 & 48666.89 & 26.9 & 332.8 & 10.0 & 2.0 \\
\hline MHV-23 & 102458 & 48666.89 & 28.9 & 330.8 & 6.0 & 3.0 \\
\hline MHV-23 & 102458 & 48666.89 & 30.9 & 328.8 & 2.0 & 2.0 \\
\hline MHV-23 & 102458 & 48666.89 & 32.9 & 326.8 & 2.0 & 1.0 \\
\hline MHV-23 & 102458 & 48666.89 & 34.9 & 324.8 & 2.0 & - \\
\hline MHV-23 & 102458 & 48666.89 & 35.9 & 323.8 & 40.0 & 10.0 \\
\hline MHV-23 & 102458 & 48666.89 & 36.9 & 322.8 & 370.0 & 110.0 \\
\hline MHV-23 & 102458 & 48666.89 & 37.9 & 321.8 & $1,190.0$ & 440.0 \\
\hline MHV-23 & 102458 & 48666.89 & 38.9 & 320.8 & 160.0 & 90.0 \\
\hline MHV-23 & 102458 & 48666.89 & 39.9 & 319.8 & 4.0 & $2,050.0$ \\
\hline MHV-23 & 102458 & 48666.89 & 40.9 & 318.8 & 20.0 & $1,500.0$ \\
\hline MHV-23 & 102458 & 48666.89 & 41.9 & 317.8 & $3,000.0$ & $1,850.0$ \\
\hline MHV-23 & 102458 & 48666.89 & 42.9 & 316.8 & $2,830.0$ & $2,280.0$ \\
\hline MHV-23 & 102458 & 48666.89 & 43.9 & 315.8 & 110.0 & 130.0 \\
\hline MHV-23 & 102458 & 48666.89 & 44.9 & 314.8 & 3.0 & 2.0 \\
\hline MHV-23 & 102458 & 48666.89 & 46.9 & 312.8 & 30.0 & 40.0 \\
\hline MHV-23 & 102458 & 48666.89 & 48.9 & 310.8 & 2.0 & - \\
\hline MHV-23 & 102458 & 48666.89 & 50.9 & 308.8 & - & 1.0 \\
\hline MHV-23 & 102458 & 48666.89 & 52.9 & 306.8 & 3.0 & 2.0 \\
\hline MHV-23 & 102458 & 48666.89 & 54.9 & 304.8 & 80.0 & 60.0 \\
\hline MHV-23 & 102458 & 48666.89 & 56.9 & 302.8 & 6.0 & 7.0 \\
\hline MHV-23 & 102458 & 48666.89 & 58.9 & 300.8 & 70.0 & 60.0 \\
\hline MHV-24 & 102439.8 & 48636.88 & 25.4 & 335.1 & 5.0 & 3.0 \\
\hline MHV-24 & 102439.8 & 48636.88 & 27.4 & 333.1 & 4.0 & 3.0 \\
\hline MHV-24 & 102439.8 & 48636.88 & 29.4 & 331.1 & 5.0 & 2.0 \\
\hline MHV-24 & 102439.8 & 48636.88 & 31.4 & 329.1 & 2.0 & - \\
\hline MHV-24 & 102439.8 & 48636.88 & 33.4 & 327.1 & 10.0 & 9.0 \\
\hline MHV-24 & 102439.8 & 48636.88 & 35.4 & 325.1 & 300.0 & 170.0 \\
\hline
\end{tabular}


WSRC-TR-2001-00077

February 2001

\begin{tabular}{|c|c|c|c|c|c|c|}
\hline ID & $\begin{array}{l}\text { SRS } \\
\text { North }\end{array}$ & $\begin{array}{l}\text { SRS } \\
\text { East }\end{array}$ & $\begin{array}{l}\text { Sample } \\
\text { Depth } \\
\mathrm{ft}\end{array}$ & $\begin{array}{c}\text { Sample } \\
\text { Elevation } \\
\mathrm{ft} \mathrm{msl}\end{array}$ & $\begin{array}{c}\text { PCE } \\
(\mu \mathrm{g} / \mathrm{kg})\end{array}$ & $\begin{array}{c}\text { TCE } \\
(\mu \mathrm{g} / \mathrm{kg})\end{array}$ \\
\hline MHV-24 & 102439.8 & 48636.88 & 36.4 & 324.1 & 30.0 & 30.0 \\
\hline MHV-24 & 102439.8 & 48636.88 & 37.4 & 323.1 & 80.0 & 80.0 \\
\hline MHV-24 & 102439.8 & 48636.88 & 38.4 & 322.1 & 30.0 & 50.0 \\
\hline MHV-24 & 102439.8 & 48636.88 & 39.4 & 321.1 & 2.0 & 3.0 \\
\hline MHV-24 & 102439.8 & 48636.88 & 40.4 & 320.1 & - & 10.0 \\
\hline MHV-24 & 102439.8 & 48636.88 & 41.4 & 319.1 & - & 50.0 \\
\hline MHV-24 & 102439.8 & 48636.88 & 42.4 & 318.1 & 440.0 & 610.0 \\
\hline MHV-24 & 102439.8 & 48636.88 & 43.4 & 317.1 & 90.0 & 90.0 \\
\hline MHV-24 & 102439.8 & 48636.88 & 44.4 & 316.1 & 3.0 & - \\
\hline MHV-24 & 102439.8 & 48636.88 & 45.4 & 315.1 & 3.0 & - \\
\hline MHV-24 & 102439.8 & 48636.88 & 47.4 & 313.1 & 5.0 & 3.0 \\
\hline MHV-24 & 102439.8 & 48636.88 & 49.4 & 311.1 & 2.0 & - \\
\hline MHV-24 & 102439.8 & 48636.88 & 51.4 & 309.1 & 4.0 & - \\
\hline MHV-24 & 102439.8 & 48636.88 & 53.4 & 307.1 & 7.0 & 2.0 \\
\hline MHV-24 & 102439.8 & 48636.88 & 55.4 & 305.1 & 20.0 & 10.0 \\
\hline MHV-24 & 102439.8 & 48636.88 & 57.4 & 303.1 & 7.0 & 4.0 \\
\hline MHV-24 & 102439.8 & 48636.88 & 59.4 & 301.1 & 40.0 & 40.0 \\
\hline MHV-25 & 102428.9 & 48680.95 & 25 & 334.1 & 9.0 & 1.0 \\
\hline MHV-25 & 102428.9 & 48680.95 & 27 & 332.1 & 70.0 & 9.0 \\
\hline MHV-25 & 102428.9 & 48680.95 & 29 & 330.1 & 10.0 & 2.0 \\
\hline MHV-25 & 102428.9 & 48680.95 & 31 & 328.1 & 60.0 & 10.0 \\
\hline MHV-25 & 102428.9 & 48680.95 & 33 & 326.1 & 90.0 & 20.0 \\
\hline MHV-25 & 102428.9 & 48680.95 & 35 & 324.1 & $1,060.0$ & 300.0 \\
\hline MHV-25 & 102428.9 & 48680.95 & 36 & 323.1 & $1,070.0$ & 440.0 \\
\hline MHV-25 & 102428.9 & 48680.95 & 37 & 322.1 & $5,290.0$ & $4,600.0$ \\
\hline MHV-25 & 102428.9 & 48680.95 & 38 & 321.1 & $9,810.0$ & $10,850.0$ \\
\hline MHV-25 & 102428.9 & 48680.95 & 39 & 320.1 & $8,210.0$ & $12,140.0$ \\
\hline MHV-25 & 102428.9 & 48680.95 & 40 & 319.1 & $3,000.0$ & $5,530.0$ \\
\hline MHV-25 & 102428.9 & 48680.95 & 41 & 318.1 & $16,440.0$ & $63,870.0$ \\
\hline MHV-25 & 102428.9 & 48680.95 & 42 & 317.1 & $10,880.0$ & $41,320.0$ \\
\hline MHV-25 & 102428.9 & 48680.95 & 43 & 316.1 & $13,940.0$ & $31,930.0$ \\
\hline MHV-25 & 102428.9 & 48680.95 & 44 & 315.1 & $27,120.0$ & $74,400.0$ \\
\hline MHV-25 & 102428.9 & 48680.95 & 45 & 314.1 & 290.0 & 450.0 \\
\hline MHV-25 & 102428.9 & 48680.95 & 47 & 312.1 & 50.0 & 80.0 \\
\hline MHV-25 & 102428.9 & 48680.95 & 49 & 310.1 & 10.0 & 40.0 \\
\hline MHV-25 & 102428.9 & 48680.95 & 51 & 308.1 & 10.0 & 20.0 \\
\hline MHV-25 & 102428.9 & 48680.95 & 53 & 306.1 & 20.0 & 10.0 \\
\hline MHV-25 & 102428.9 & 48680.95 & 55 & 304.1 & 30.0 & 30.0 \\
\hline MHV-25 & 102428.9 & 48680.95 & 57 & 302.1 & 20.0 & 10.0 \\
\hline MHV-25 & 102428.9 & 48680.95 & 59 & 300.1 & 350.0 & 750.0 \\
\hline MHV-26 & 102404.2 & 48655.31 & 24.9 & 334.9 & 7.0 & 10.0 \\
\hline MHV-26 & 102404.2 & 48655.31 & 26.9 & 332.9 & 60.0 & 50.0 \\
\hline MHV-26 & 102404.2 & 48655.31 & 28.9 & 330.9 & 60.0 & 50.0 \\
\hline MHV-26 & 102404.2 & 48655.31 & 30.9 & 328.9 & 10.0 & 10.0 \\
\hline MHV-26 & 102404.2 & 48655.31 & 31.9 & 327.9 & 140.0 & 60.0 \\
\hline MHV-26 & 102404.2 & 48655.31 & 32.9 & 326.9 & 690.0 & 200.0 \\
\hline
\end{tabular}


WSRC-TR-2001-00077

February 2001

\begin{tabular}{|c|c|c|c|c|c|c|}
\hline ID & $\begin{array}{l}\text { SRS } \\
\text { North }\end{array}$ & $\begin{array}{l}\text { SRS } \\
\text { East }\end{array}$ & $\begin{array}{c}\text { Sample } \\
\text { Depth } \\
\mathrm{ft}\end{array}$ & $\begin{array}{c}\text { Sample } \\
\text { Elevation } \\
\mathrm{ft} \text { msl }\end{array}$ & $\begin{array}{c}\text { PCE } \\
(\mu \mathrm{g} / \mathrm{kg})\end{array}$ & $\begin{array}{c}\text { TCE } \\
(\mu \mathrm{g} / \mathrm{kg})\end{array}$ \\
\hline MHV-26 & 102404.2 & 48655.31 & 34.9 & 324.9 & $1,100.0$ & 420.0 \\
\hline MHV-26 & 102404.2 & 48655.31 & 35.9 & 323.9 & $1,490.0$ & 600.0 \\
\hline MHV-26 & 102404.2 & 48655.31 & 36.9 & 322.9 & $3,070.0$ & $1,560.0$ \\
\hline MHV-26 & 102404.2 & 48655.31 & 37.9 & 321.9 & $2,810.0$ & $1,550.0$ \\
\hline MHV-26 & 102404.2 & 48655.31 & 38.9 & 320.9 & $2,520.0$ & $2,540.0$ \\
\hline MHV-26 & 102404.2 & 48655.31 & 39.9 & 319.9 & $2,240.0$ & $3,070.0$ \\
\hline MHV-26 & 102404.2 & 48655.31 & 40.9 & 318.9 & $3,320.0$ & $5,700.0$ \\
\hline MHV-26 & 102404.2 & 48655.31 & 41.9 & 317.9 & $2,220.0$ & $2,920.0$ \\
\hline MHV-26 & 102404.2 & 48655.31 & 42.9 & 316.9 & $1,640.0$ & $1,470.0$ \\
\hline MHV-26 & 102404.2 & 48655.31 & 43.9 & 315.9 & 230.0 & 310.0 \\
\hline MHV-26 & 102404.2 & 48655.31 & 44.9 & 314.9 & 580.0 & 880.0 \\
\hline MHV-26 & 102404.2 & 48655.31 & 46.9 & 312.9 & 30.0 & 30.0 \\
\hline MHV-26 & 102404.2 & 48655.31 & 48.9 & 310.9 & 30.0 & 40.0 \\
\hline MHV-26 & 102404.2 & 48655.31 & 50.9 & 308.9 & 10.0 & 10.0 \\
\hline MHV-26 & 102404.2 & 48655.31 & 52.9 & 306.9 & 20.0 & 20.0 \\
\hline MHV-26 & 102404.2 & 48655.31 & 54.9 & 304.9 & 30.0 & 40.0 \\
\hline MHV-26 & 102404.2 & 48655.31 & 56.9 & 302.9 & 20.0 & 20.0 \\
\hline MHV-26 & 102404.2 & 48655.31 & 58.9 & 300.9 & 40.0 & 40.0 \\
\hline MHV-27 & 102374.3 & 48654.5 & 26.9 & 333 & 5.0 & 2.0 \\
\hline MHV-27 & 102374.3 & 48654.5 & 28.9 & 331 & 4.0 & - \\
\hline MHV-27 & 102374.3 & 48654.5 & 30.9 & 329 & 10.0 & 10.0 \\
\hline MHV-27 & 102374.3 & 48654.5 & 32.9 & 327 & 30.0 & 5.0 \\
\hline MHV-27 & 102374.3 & 48654.5 & 34.9 & 325 & 200.0 & 40.0 \\
\hline MHV-27 & 102374.3 & 48654.5 & 35.9 & 324 & 370.0 & 80.0 \\
\hline MHV-27 & 102374.3 & 48654.5 & 36.9 & 323 & 90.0 & 30.0 \\
\hline MHV-27 & 102374.3 & 48654.5 & 37.9 & 322 & 4.0 & 2.0 \\
\hline MHV-27 & 102374.3 & 48654.5 & 38.9 & 321 & 180.0 & 110.0 \\
\hline MHV-27 & 102374.3 & 48654.5 & 39.9 & 320 & 370.0 & 200.0 \\
\hline MHV-27 & 102374.3 & 48654.5 & 40.9 & 319 & 710.0 & 360.0 \\
\hline MHV-27 & 102374.3 & 48654.5 & 41.9 & 318 & 220.0 & 120.0 \\
\hline MHV-27 & 102374.3 & 48654.5 & 42.9 & 317 & 30.0 & 20.0 \\
\hline MHV-27 & 102374.3 & 48654.5 & 43.9 & 316 & 570.0 & 270.0 \\
\hline MHV-27 & 102374.3 & 48654.5 & 44.9 & 315 & 3.0 & 2.0 \\
\hline MHV-27 & 102374.3 & 48654.5 & 46.9 & 313 & 20.0 & 20.0 \\
\hline MHV-27 & 102374.3 & 48654.5 & 48.9 & 311 & 20.0 & 20.0 \\
\hline MHV-27 & 102374.3 & 48654.5 & 50.9 & 309 & 10.0 & 10.0 \\
\hline MHV-27 & 102374.3 & 48654.5 & 52.9 & 307 & 10.0 & 10.0 \\
\hline MHV-27 & 102374.3 & 48654.5 & 54.9 & 305 & 9.0 & 10.0 \\
\hline MHV-27 & 102374.3 & 48654.5 & 56.9 & 303 & 1.0 & - \\
\hline MHV-27 & 102374.3 & 48654.5 & 58.9 & 301 & 4.0 & 3.0 \\
\hline MHV-28 & 102366.3 & 48647.61 & 25 & 334.9 & 140.0 & 7.0 \\
\hline MHV-28 & 102366.3 & 48647.61 & 27 & 332.9 & 160.0 & 10.0 \\
\hline MHV-28 & 102366.3 & 48647.61 & 29 & 330.9 & 7.0 & 2.0 \\
\hline MHV-28 & 102366.3 & 48647.61 & 31 & 328.9 & 3.0 & 3.0 \\
\hline MHV-28 & 102366.3 & 48647.61 & 33 & 326.9 & 10.0 & 10.0 \\
\hline MHV-28 & 102366.3 & 48647.61 & 35 & 324.9 & 350.0 & 50.0 \\
\hline
\end{tabular}


WSRC-TR-2001-00077

February 2001

\begin{tabular}{|c|c|c|c|c|c|c|}
\hline ID & $\begin{array}{l}\text { SRS } \\
\text { North }\end{array}$ & $\begin{array}{l}\text { SRS } \\
\text { East }\end{array}$ & $\begin{array}{l}\text { Sample } \\
\text { Depth } \\
\mathrm{ft}\end{array}$ & $\begin{array}{c}\text { Sample } \\
\text { Elevation } \\
\mathrm{ft} \text { msl }\end{array}$ & $\begin{array}{c}\text { PCE } \\
(\mu \mathrm{g} / \mathrm{kg})\end{array}$ & $\begin{array}{c}\text { TCE } \\
(\mu \mathrm{g} / \mathrm{kg})\end{array}$ \\
\hline MHV-28 & 102366.3 & 48647.61 & 36 & 323.9 & 170.0 & 20.0 \\
\hline MHV-28 & 102366.3 & 48647.61 & 37 & 322.9 & 100.0 & 10.0 \\
\hline MHV-28 & 102366.3 & 48647.61 & 38 & 321.9 & $1,030.0$ & 80.0 \\
\hline MHV-28 & 102366.3 & 48647.61 & 39 & 320.9 & 880.0 & 60.0 \\
\hline MHV-28 & 102366.3 & 48647.61 & 40 & 319.9 & $1,300.0$ & 90.0 \\
\hline MHV-28 & 102366.3 & 48647.61 & 41 & 318.9 & 90.0 & 40.0 \\
\hline MHV-28 & 102366.3 & 48647.61 & 42 & 317.9 & 4.0 & 2.0 \\
\hline MHV-28 & 102366.3 & 48647.61 & 43 & 316.9 & 230.0 & 110.0 \\
\hline MHV-28 & 102366.3 & 48647.61 & 44 & 315.9 & 140.0 & 80.0 \\
\hline MHV-28 & 102366.3 & 48647.61 & 45 & 314.9 & 190.0 & 120.0 \\
\hline MHV-28 & 102366.3 & 48647.61 & 47 & 312.9 & 10.0 & 10.0 \\
\hline MHV-28 & 102366.3 & 48647.61 & 49 & 310.9 & 10.0 & 10.0 \\
\hline MHV-28 & 102366.3 & 48647.61 & 51 & 308.9 & 50.0 & 50.0 \\
\hline MHV-28 & 102366.3 & 48647.61 & 53 & 306.9 & 7.0 & 8.0 \\
\hline MHV-28 & 102366.3 & 48647.61 & 55 & 304.9 & 4.0 & 5.0 \\
\hline MHV-28 & 102366.3 & 48647.61 & 57 & 302.9 & 10.0 & 10.0 \\
\hline MHV-29 & 102362.2 & 48661.78 & 25.9 & 333.7 & 420.0 & 40.0 \\
\hline MHV-29 & 102362.2 & 48661.78 & 27.9 & 331.7 & 440.0 & 40.0 \\
\hline MHV-29 & 102362.2 & 48661.78 & 29.9 & 329.7 & 70.0 & 10.0 \\
\hline MHV-29 & 102362.2 & 48661.78 & 31.9 & 327.7 & 50.0 & 20.0 \\
\hline MHV-29 & 102362.2 & 48661.78 & 33.9 & 325.7 & $1,020.0$ & 120.0 \\
\hline MHV-29 & 102362.2 & 48661.78 & 35.9 & 323.7 & 680.0 & 190.0 \\
\hline MHV-29 & 102362.2 & 48661.78 & 36.9 & 322.7 & $1,560.0$ & 550.0 \\
\hline MHV-29 & 102362.2 & 48661.78 & 37.9 & 321.7 & 940.0 & 580.0 \\
\hline MHV-29 & 102362.2 & 48661.78 & 38.9 & 320.7 & $1,170.0$ & 790.0 \\
\hline MHV-29 & 102362.2 & 48661.78 & 39.9 & 319.7 & 700.0 & 530.0 \\
\hline MHV-29 & 102362.2 & 48661.78 & 40.9 & 318.7 & 750.0 & 590.0 \\
\hline MHV-29 & 102362.2 & 48661.78 & 41.9 & 317.7 & 590.0 & 410.0 \\
\hline MHV-29 & 102362.2 & 48661.78 & 42.9 & 316.7 & 580.0 & 300.0 \\
\hline MHV-29 & 102362.2 & 48661.78 & 43.9 & 315.7 & 190.0 & 120.0 \\
\hline MHV-29 & 102362.2 & 48661.78 & 44.9 & 314.7 & 170.0 & 120.0 \\
\hline MHV-29 & 102362.2 & 48661.78 & 47.9 & 311.7 & 50.0 & 50.0 \\
\hline MHV-29 & 102362.2 & 48661.78 & 49.9 & 309.7 & 5.0 & 6.0 \\
\hline MHV-29 & 102362.2 & 48661.78 & 51.9 & 307.7 & 9.0 & 10.0 \\
\hline MHV-29 & 102362.2 & 48661.78 & 53.9 & 305.7 & 10.0 & 10.0 \\
\hline MHV-29 & 102362.2 & 48661.78 & 55.9 & 303.7 & 10.0 & 10.0 \\
\hline MHV-29 & 102362.2 & 48661.78 & 57.9 & 301.7 & 50.0 & 70.0 \\
\hline MHV-29 & 102362.2 & 48661.78 & 59.9 & 299.7 & 20.0 & 30.0 \\
\hline
\end{tabular}




\section{Appendix E - Boring and Well Coordinates}

Table E1 - CPT Boring Coordinates

\begin{tabular}{|l|r|r|r|r|r|}
\hline $\begin{array}{l}\text { CPT } \\
\text { Boring }\end{array}$ & SRS North & SRS East & $\begin{array}{l}\text { Ground } \\
\text { Elevation } \\
\text { ft msl }\end{array}$ & $\begin{array}{l}\text { Total } \\
\text { Depth } \\
\text { ft }\end{array}$ & $\begin{array}{l}\text { lnstall } \\
\text { Date }\end{array}$ \\
\hline MVB-101 & 103639.90 & 49005.01 & 366.38 & 104 & $11 / 14 / 00$ \\
\hline MVB-104 & 103440.76 & 49089.99 & 363.68 & 103 & $11 / 29 / 00$ \\
\hline MVB-105 & 103425.06 & 49005.00 & 363.79 & 103 & $11 / 27 / 00$ \\
\hline MVB-106 & 103262.38 & 49139.32 & 361.71 & 102 & $11 / 27 / 00$ \\
\hline MVB-107 & 103249.92 & 48980.02 & 362.23 & 102 & $11 / 22 / 00$ \\
\hline MVB-108 & 103086.91 & 48930.88 & 363.57 & 102 & $11 / 28 / 00$ \\
\hline MVB-109 & 103069.98 & 49060.04 & 365.08 & 121 & $11 / 7 / 00$ \\
\hline MVB-110 & 102952.18 & 48878.96 & 365.53 & 100 & $11 / 15 / 00$ \\
\hline MVB-113 & 102854.07 & 48958.94 & 369.16 & 102 & $11 / 28 / 00$ \\
\hline MVB-114 & 102802.72 & 48821.55 & 365.08 & 111 & $11 / 15 / 00$ \\
\hline MVB-117 & 102778.24 & 48989.67 & 367.14 & 103 & $11 / 16 / 00$ \\
\hline MVB-118 & 102719.96 & 48649.96 & 363.48 & 98 & $11 / 22 / 00$ \\
\hline MVB-119 & 102709.96 & 48749.99 & 362.72 & 102 & $11 / 16 / 00$ \\
\hline MVB-120 & 102690.01 & 48874.99 & 361.77 & 98 & $11 / 29 / 00$ \\
\hline MVB-121 & 102610.03 & 48859.99 & 356.66 & 100 & $11 / 21 / 00$ \\
\hline MVB-122 & 102610.02 & 48720.00 & 360.26 & 82 & $11 / 17 / 00$ \\
\hline MVB-124 & 102485.02 & 48670.00 & 359.73 & 92 & $11 / 17 / 00$ \\
\hline MVB-125 & 102437.46 & 48723.27 & 356.99 & 103 & $11 / 21 / 00$ \\
\hline MVB-126 & 102407.49 & 48627.90 & 360.37 & 110 & $11 / 20 / 00$ \\
\hline MVB-129 & 102249.93 & 48600.07 & 360.42 & 129 & $11 / 20 / 00$ \\
\hline
\end{tabular}


Table E2 - Previous Sediment Boring and Well Coordinates

\begin{tabular}{|c|c|c|c|c|c|c|c|c|c|}
\hline ID & $\begin{array}{l}\text { SRS } \\
\text { North }\end{array}$ & $\begin{array}{l}\text { SRS } \\
\text { East }\end{array}$ & \begin{tabular}{|l|} 
Screen \\
Top \\
ft msl
\end{tabular} & $\begin{array}{l}\text { Screen } \\
\text { Bottom } \\
\mathrm{ft} \mathrm{msl}\end{array}$ & $\begin{array}{l}\text { Ground } \\
\text { Elevation } \\
\mathrm{ft} \text { msl }\end{array}$ & \begin{tabular}{|l} 
Effective \\
Depth \\
$\mathrm{ft}$
\end{tabular} & $\begin{array}{l}\text { Diam. } \\
\text { in }\end{array}$ & Material & $\begin{array}{l}\text { Install } \\
\text { Date }\end{array}$ \\
\hline MHT 1C & 102706.8 & 48765.6 & \begin{tabular}{|l|}
209.7 \\
\end{tabular} & 204.7 & 362.7 & \begin{tabular}{|l|}
160.2 \\
\end{tabular} & & 4 PVC & $6 / 7 / 90$ \\
\hline MHT 2C & 102747.1 & 48780.3 & 212.1 & 207.1 & 364.1 & 159.1 & & 4 PVC & $4 / 6 / 90$ \\
\hline MHT 3C & 102704.3 & 48861.1 & 209.6 & 204.6 & 362.6 & 160.2 & & 4 PVC & $4 / 23 / 90$ \\
\hline MHT $4 \mathrm{C}$ & \begin{tabular}{|l|}
102778.9 \\
\end{tabular} & 48863.5 & 213.4 & 208.4 & 367.4 & 161.1 & & 4 PVC & $4 / 12 / 90$ \\
\hline MHT 5C & 102725.1 & 48905.9 & 210.1 & 205.1 & 364.1 & 161.1 & & 4 PVC & $5 / 29 / 90$ \\
\hline MHT 6C & 102810.8 & 48900 & 212.6 & 207.6 & 369.6 & 164.1 & & 4 PVC & $4 / 26 / 90$ \\
\hline MHT 7C & 102788.9 & 48977.5 & 212 & 207 & 368 & 163 & & 4 PVC & $6 / 21 / 90$ \\
\hline MHT $8 \mathrm{C}$ & 102880.7 & 48970.2 & 212.3 & 207.3 & 369.3 & 164.2 & & 4 PVC & $6 / 5 / 90$ \\
\hline MHT 9C & 102814.4 & 49015.6 & 214.7 & 209.7 & 367.7 & 159.9 & & 4 PVC & $6 / 15 / 90$ \\
\hline MHT 10C & 102892.3 & 49011.6 & 211.9 & 206.9 & 368.9 & 163.8 & & $4 \mathrm{PVC}$ & $6 / 19 / 90$ \\
\hline MHT 11C & 102854 & 48846.1 & 214.2 & 209.2 & 366.2 & 159.1 & & 4 PVC & $6 / 4 / 91$ \\
\hline MHT 12C & 102844.8 & 49061.7 & 214.4 & 209.4 & 367.9 & 160.8 & & 4 PVC & $6 / 13 / 91$ \\
\hline MHT 15C & 102520.7 & 48741.9 & 204.5 & 199.7 & 357.7 & 159.8 & & 4 PVC & $5 / 4 / 92$ \\
\hline MHT 16C & 102430.9 & 48672.8 & 205.5 & 200.7 & 359.5 & 160.9 & & 4 PVC & $5 / 20 / 92$ \\
\hline MHT 17C & 102394.6 & 48706.9 & 205.7 & 200.8 & 357.7 & 158.9 & & 4 PVC & $6 / 18 / 92$ \\
\hline MHT 18C & 102486.1 & 48650.9 & 205.3 & 200.5 & 360.3 & 161.7 & & 4 PVC & $6 / 4 / 92$ \\
\hline MHT 19C & 102502.7 & 48699.1 & 205.9 & 201.1 & 358.9 & 159.8 & & 4 PVC & $5 / 12 / 92$ \\
\hline MHT 20C & 102589.3 & 48710.8 & 206.5 & 201.6 & 360.3 & 160.8 & & 4 PVC & $6 / 19 / 92$ \\
\hline MHV $1 \mathrm{~A}$ & 102749.3 & 48842 & 270.6 & 265.6 & 365.6 & 102 & & 1 PVC & $5 / 9 / 90$ \\
\hline MHV 1B & \begin{tabular}{|l|}
102749.3 \\
\end{tabular} & 48842 & 295.6 & 290.6 & 365.6 & 77 & & 1 PVC & $5 / 10 / 90$ \\
\hline MHV 1C & 102749.3 & 48842 & 321.6 & 315.6 & 365.6 & 52 & & 1 PVC & $5 / 11 / 90$ \\
\hline MHV 2A & 102755.9 & 48903.2 & 271.4 & 266.4 & 366.4 & 101.8 & & 1 PVC & $5 / 29 / 90$ \\
\hline MHV 2B & 102755.9 & 48903.2 & 296.4 & 291.4 & 366.4 & 76.8 & & 1 PVC & $5 / 30 / 90$ \\
\hline MHV 2C & \begin{tabular}{|l|}
102755.9 \\
\end{tabular} & 48903.2 & 322.4 & 317.4 & 366.4 & 50.8 & & 1 PVC & $5 / 31 / 90$ \\
\hline MHV 3A & 102774.7 & 48874.1 & 273.2 & 268.2 & 368.2 & 102.1 & & 1 PVC & $5 / 14 / 90$ \\
\hline MHV 3B & 102774.7 & 48874.1 & 298.8 & 293.8 & 368.2 & 76.5 & & $1 \mathrm{PVC}$ & $5 / 15 / 90$ \\
\hline MHV 3C & 102774.7 & 48874.1 & 323.2 & 318.2 & 368.2 & 52.1 & & $1 \mathrm{PVC}$ & $5 / 16 / 90$ \\
\hline MHV 4A & \begin{tabular}{|l|}
102841.7 \\
\end{tabular} & 48842.5 & 272.1 & 267.1 & 366.1 & 101.5 & & 1 PVC & $6 / 13 / 90$ \\
\hline $\mathrm{MHV}$ 4B & 102841.7 & 48842.5 & 296.1 & 291.1 & 366.1 & 77.4 & & 1 PVC & $6 / 15 / 90$ \\
\hline MHV 4C & 102841.7 & 48842.5 & 323.6 & 318.6 & 366.1 & 50.1 & & $1 \mathrm{PVC}$ & $6 / 18 / 90$ \\
\hline MHV 5A & 102878.8 & 48917.5 & 284.2 & 279.2 & 369.2 & 92.4 & & 1 PVC & $6 / 6 / 90$ \\
\hline MHV 5B & 102878.8 & 48917.5 & 301.2 & 296.2 & 369.2 & 75.3 & & 1 PVC & $6 / 7 / 90$ \\
\hline MHV 5C & 102878.8 & 48917.5 & 324.2 & 319.2 & 369.2 & 52.4 & & $1 \mathrm{PVC}$ & $6 / 8 / 90$ \\
\hline MHV 6 & 102780.1 & 48891.5 & 358.8 & 253.8 & 368.1 & 114.6 & & 4 PVC & $6 / 25 / 91$ \\
\hline MHV 7 & 102811.5 & 48878.1 & 358.2 & 253.2 & 368.2 & 117.1 & & 4 PVC & $6 / 21 / 91$ \\
\hline \multirow[t]{7}{*}{ MHV 8} & \multirow[t]{7}{*}{102610.3} & \multirow[t]{7}{*}{48846.3} & 323.4 & 321.1 & \multirow[t]{7}{*}{357.7} & \multirow[t]{7}{*}{117.4} & & \multirow[t]{7}{*}{4 PVC } & \multirow[t]{7}{*}{ 4/10/92 } \\
\hline & & & 303.8 & 301.5 & & & & & \\
\hline & & & 296.5 & 294.2 & & & & & \\
\hline & & & 285.2 & 282.9 & & & & & \\
\hline & & & 268.9 & 266.7 & & & & & \\
\hline & & & 254.7 & 252.4 & & & & & \\
\hline & & & 243.5 & 241.2 & & & & & \\
\hline
\end{tabular}




\begin{tabular}{|c|c|c|c|c|c|c|c|c|c|}
\hline ID & \begin{tabular}{|l} 
SRS \\
North
\end{tabular} & $\begin{array}{l}\text { SRS } \\
\text { East }\end{array}$ & $\begin{array}{l}\text { Screen } \\
\text { Top } \\
\mathrm{ft} \mathrm{msl}\end{array}$ & $\begin{array}{l}\text { Screen } \\
\text { Bottom } \\
\mathrm{ft} \mathrm{msl}\end{array}$ & $\begin{array}{l}\text { Ground } \\
\text { Elevation } \\
\mathrm{ft} \text { msl }\end{array}$ & \begin{tabular}{|l} 
Effective \\
Depth \\
ft
\end{tabular} & $\begin{array}{l}\text { Diam } \\
\text { in }\end{array}$ & Material & $\begin{array}{l}\text { Install } \\
\text { Date }\end{array}$ \\
\hline \multirow[t]{8}{*}{ MHV 9} & \multirow[t]{8}{*}{102830.1} & \multirow[t]{8}{*}{49046.7} & 343.5 & 341.2 & \multirow{8}{*}{\begin{tabular}{|l}
367.7 \\
\\
\\
\end{tabular}} & \multirow[t]{8}{*}{123.6} & \multirow{8}{*}{\multicolumn{2}{|c|}{4 PVC }} & \multirow[t]{8}{*}{$4 / 16 / 92$} \\
\hline & & & 318.8 & 316.5 & & & & & \\
\hline & & & 303.5 & 301.2 & & & & & \\
\hline & & & 292.2 & 290 & & & & & \\
\hline & & & 281 & 278.7 & & & & & \\
\hline & & & 268.7 & 266.4 & & & & & \\
\hline & & & 255.3 & 253 & & & & & \\
\hline & & & 248 & 245.7 & & & & & \\
\hline MHV 20A & 102418.4 & 48658.3 & 311.6 & 306.7 & 359.6 & 55.1 & & 1 PVC & $12 / 4 / 92$ \\
\hline MHV 20B & 102418.4 & 48658.3 & 321.6 & 316.7 & 359.6 & 45 & & 1) PVC & $12 / 4 / 92$ \\
\hline MHV 20C & 102418.4 & 48658.3 & 331.6 & 326.7 & 359.6 & 35 & & $1 \mathrm{PVC}$ & $12 / 4 / 92$ \\
\hline MHV 21A & 102433.6 & 48661.5 & 311.7 & 306.8 & 359.7 & 55.2 & & 1 PVC & $12 / 4 / 92$ \\
\hline MHV 21B & 102433.6 & 48661.5 & 321.7 & 316.8 & 359.7 & 45.2 & & $1 \mathrm{PVC}$ & $12 / 4 / 92$ \\
\hline MHV 21C & 102433.6 & 48661.5 & 331.7 & 326.8 & 359.7 & 35.2 & & 1) PVC & $12 / 4 / 92$ \\
\hline MHV 22A & 102443.5 & 48663.8 & 311.7 & 306.8 & 359.7 & 55.2 & & 1 PVC & $12 / 4 / 92$ \\
\hline MHV 22B & 102433.5 & 48663.8 & 321.7 & 316.8 & 359.7 & 45.2 & & 1 PVC & $12 / 4 / 92$ \\
\hline MHV 22C & 102433.5 & 48663.8 & 331.7 & 326.8 & 359.7 & 35.2 & & 1 PVC & $12 / 4 / 92$ \\
\hline MHV 23A & 102458 & 48666.9 & 311.7 & 306.8 & 359.7 & 55.1 & & 1 PVC & $12 / 4 / 92$ \\
\hline MHV 23B & 102458 & 48666.9 & 321.7 & 316.8 & 359.7 & 45.1 & & 1 PVC & $12 / 4 / 92$ \\
\hline MHV 23C & 102458 & 48666.9 & 331.7 & 326.8 & 359.7 & 35.2 & & 1 PVC & $12 / 4 / 92$ \\
\hline MHV 24A & \begin{tabular}{|l|}
102439.8 \\
\end{tabular} & 48636.9 & 312.5 & 307.6 & 360.5 & 55.1 & & 1 PVC & $12 / 4 / 92$ \\
\hline MHV 24B & 102439.8 & 48636.9 & 322.5 & 317.6 & 360.5 & 45.1 & & $1 \mathrm{PVC}$ & $12 / 4 / 92$ \\
\hline MHV 24C & 102439.8 & 48636.9 & 332.5 & 327.6 & 360.5 & 35.2 & & 1 PVC & $12 / 4 / 92$ \\
\hline MHV 25A & 102428.9 & 48681 & 311.1 & 306.2 & 359.1 & 55.3 & & 1 PVC & $12 / 4 / 92$ \\
\hline MHV 25B & \begin{tabular}{|l|}
102428.9 \\
\end{tabular} & 48681 & 321.1 & 316.2 & 359.1 & 45.3 & & 1 PVC & $12 / 4 / 92$ \\
\hline MHV 25C & 102428.9 & 48681 & 331.1 & 326.2 & 359.1 & 35.3 & & 1) PVC & $12 / 4 / 92$ \\
\hline MHV 26A & 102404.2 & 48655.3 & 311.8 & 306.9 & 359.8 & 55.2 & & 1 PVC & $12 / 4 / 92$ \\
\hline MHV 26B & 102404.2 & 48655.3 & 321.8 & 316.9 & 359.8 & 45.2 & & $1 \mathrm{PVC}$ & $12 / 4 / 92$ \\
\hline MHV 26C & \begin{tabular}{|l|}
102404.2 \\
\end{tabular} & 48655.3 & 331.8 & 326.9 & 359.8 & 35.2 & & 1 PVC & $12 / 4 / 92$ \\
\hline MHV 27A & 102374.3 & 48654.5 & 311.9 & 307 & 359.9 & 55.1 & & 1 PVC & $12 / 4 / 92$ \\
\hline MHV 27B & 102374.3 & 48654.5 & 321.9 & 317 & 359.9 & 45 & & 1 PVC & $12 / 4 / 92$ \\
\hline MHV 27C & 102374.3 & 48654.5 & 331.9 & 327 & 359.9 & 35.1 & & 1 PVC & $12 / 4 / 92$ \\
\hline MHV 28A & 102366.3 & 48647.6 & 311.9 & 307 & 359.9 & 55.2 & & 1 PVC & $12 / 4 / 92$ \\
\hline MHV 28B & 102366.3 & 48647.6 & 321.9 & 317 & 359.9 & 45.2 & & 1 PVC & $12 / 4 / 92$ \\
\hline MHV 28C & 102366.3 & 48647.6 & 331.9 & 327 & 359.9 & 35.2 & & 1 PVC & $12 / 4 / 92$ \\
\hline MHV 29A & 102362.2 & 48661.8 & 311.6 & 306.7 & 359.6 & 55.1 & & 1 PVC & $12 / 4 / 92$ \\
\hline MHV 29B & 102362.2 & 48661.8 & 321.6 & 316.7 & 359.6 & 45.1 & & 1 PVC & $12 / 4 / 92$ \\
\hline MHV 29C & 102362.2 & 48661.8 & 331.6 & 326.7 & 359.6 & 35.1 & & 1PVC & $12 / 4 / 92$ \\
\hline
\end{tabular}


Table E3 - Vadose Zone Horizontal Well Coordinates

\begin{tabular}{|c|c|c|c|c|c|c|c|c|}
\hline \multicolumn{3}{|l|}{ AMH-2 } & \multicolumn{3}{|l|}{$\overline{\mathrm{AMH}-4}$} & \multicolumn{3}{|l|}{ AMH-5 } \\
\hline $\begin{array}{l}\text { SRS } \\
\text { North }\end{array}$ & $\begin{array}{l}\text { SRS } \\
\text { East }\end{array}$ & $\begin{array}{l}\text { Elevation } \\
\mathrm{ft} \mathrm{msl}\end{array}$ & $\begin{array}{l}\text { SRS } \\
\text { North }\end{array}$ & $\begin{array}{l}\text { SRS } \\
\text { East }\end{array}$ & $\begin{array}{l}\text { Elevation } \\
\mathrm{ft} \mathrm{msl}\end{array}$ & $\begin{array}{l}\text { SRS } \\
\text { North }\end{array}$ & $\begin{array}{l}\text { SRS } \\
\text { East }\end{array}$ & $\begin{array}{l}\text { Elevation } \\
\mathrm{ft} \mathrm{msl}\end{array}$ \\
\hline 102708.8 & 48807.81 & 363.04 & 102670.5 & 48779.61 & 362.1 & 102207.3 & 48604.04 & 358.3893 \\
\hline 102708.8 & 48807.81 & 10.04 & 102665.6 & 48776.76 & 341.89 & 102216.5 & 48606.38 & 357.2769 \\
\hline 102708.8 & 48807.82 & 8.04 & 102664.9 & 4877 & 9.01 & 102227.6 & 4860 & .3801 \\
\hline 102708.8 & 48807.83 & 36.04 & 102664.2 & 48775.88 & 6.14 & 102236.9 & 48611.21 & 1.7034 \\
\hline 102708.9 & 48807.86 & 334.04 & 102663.4 & 48775.51 & 33.26 & 102245.2 & 48612.31 & .0382 \\
\hline 102709.1 & 48807.95 & 332.05 & 102662.5 & 48775.21 & 30.39 & 102254.5 & 48613.69 & .3615 \\
\hline 102709.4 & 48808.08 & 330.08 & 102661.7 & 48774.88 & 27.53 & 102264.7 & 486 & 4647 \\
\hline 102709.8 & 48808.27 & 328.14 & 102660.8 & 48774.52 & 324.69 & 102274.9 & 486 & 3476 \\
\hline 102710.3 & 48808.49 & 326.22 & 102660.2 & 48774.24 & 322.8 & 102283.2 & 18.7 & 7.219 \\
\hline 102711 & 48808.76 & 324.34 & 102659.3 & 48773.78 & 320 & 102292.5 & 48620.34 & 334.3221 \\
\hline 102711.7 & 48809.08 & 2.51 & 102658.2 & 48773.28 & 7.22 & 102301.8 & 4862 & 8772 \\
\hline 102712.5 & 4880 & 320.72 & 102657.2 & 4877 & 4.48 & 102311 & & 9803 \\
\hline 102713.4 & 48809.83 & 318.98 & 102656 & 48772.08 & 11.79 & 102321.2 & 4862 & 5238 \\
\hline 102714.4 & 48810.26 & 317.3 & 102654.8 & 48771.4 & 309.13 & 102330.5 & 4862 & .7509 \\
\hline 102715.5 & 4881 & 315.7 & 102653.5 & 48770.7 & 6.52 & 102339.7 & 486 & 1866 \\
\hline 102716.7 & 4881 & 314.15 & 102652.6 & 48770.21 & 04.8 & 102349.9 & 486 & .854 \\
\hline 102717.9 & 48811.77 & 312.68 & 102651.2 & 48769.4 & 302.28 & 102360.1 & 4863 & 9618 \\
\hline 102719.3 & 48812.34 & 311.28 & 102649.7 & 48768.51 & 299.81 & 102370.3 & 486 & 5099 \\
\hline 102720.7 & 4881 & & 102648.2 & 487 & 97.4 & 102379.6 & 486 & 176 \\
\hline 102722.1 & 4881 & 308.76 & 102646.5 & 4876 & 95.06 & 102389.7 & 486 & 3975 \\
\hline 102723.6 & 48814.22 & 07.63 & 102644.8 & 4876 & 2.78 & 102399 & 486 & 3975 \\
\hline 102725.2 & 48814.9 & 6.58 & 102643.1 & 4876 & 0.57 & 102408.3 & 486 & 3975 \\
\hline 102726.8 & 488 & & 102641.3 & 4876 & 88.42 & 102417.5 & 486 & 176 \\
\hline 102728.5 & 48816 & 304.79 & 102639.4 & 48762.53 & 86.34 & 102427.7 & 486 & 6176 \\
\hline 102730.2 & 48817.06 & 4.04 & 102637.5 & 48761.33 & 4.34 & 102437.9 & 486 & 8378 \\
\hline 102731.9 & 48817.81 & & 102635.6 & 4876 & & 102447.2 & 4864 & 695 \\
\hline 102733.7 & 4881 & & 102633.5 & 48758.93 & 0.59 & 102457.4 & 486 & 0695 \\
\hline 102735.4 & 48819.39 & 302.44 & 102631.4 & 48757.71 & 278.82 & 102466.7 & 48650.87 & .8378 \\
\hline 102737.2 & 48820.21 & 2.11 & 102629.3 & 48756.45 & 7.12 & 102476.8 & 4865 & 8378 \\
\hline 102739.1 & 48821.02 & & 102627.1 & 48755.16 & .51 & 102486.1 & 4865 & 3378 \\
\hline 102740.9 & 48821.85 & 301.75 & 102624.9 & 48753.82 & & 102496.3 & 48655.86 & 8378 \\
\hline 102749.1 & 48825.42 & 301.4 & 102622.7 & 48752.44 & 272.55 & 102505.5 & 48657.57 & .8378 \\
\hline 102758.3 & 48829.29 & 1.03 & 102620.4 & 48751.02 & 1.21 & 102514.8 & 4865 & 8378 \\
\hline 102767.5 & 48833.27 & 0.57 & 102618.1 & 48749.59 & 69.95 & 102525.9 & 4866 & 2897 \\
\hline 102776.7 & 48837 & & 102615.7 & 48748.15 & 268.77 & 102535.2 & 4866 & 5099 \\
\hline 102785.8 & 48841.34 & 299.48 & 102613.4 & 48746.7 & 267.66 & 102544.5 & 3.4 & 5099 \\
\hline 102794.7 & 48845.7 & 8.85 & 102611 & 48745.24 & 266.6 & 102555.6 & 4866 & 320.5099 \\
\hline 102803.7 & 48850.12 & 298.13 & 102608.6 & 48743.77 & 265.59 & 102564.8 & 48666.79 & 320.73 \\
\hline 102812.6 & 48854.55 & 297.3 & 102606.2 & 48742.2 & 264.63 & 102574.1 & 48668.47 & 321.4021 \\
\hline 102821.5 & 48858.97 & 296.34 & 102603.8 & 48740.54 & 263.76 & 102584.3 & 48670.06 & 321.6223 \\
\hline
\end{tabular}


WSRC-TR-2001-00077

February 2001

Table E3 - Horizontal Well Coordinates (continued)

\begin{tabular}{|c|c|c|c|c|c|c|c|c|}
\hline \multicolumn{3}{|l|}{$\mathrm{AMH}-2$} & \multicolumn{3}{|l|}{ AMH-4 } & \multicolumn{3}{|l|}{ AMH-5 } \\
\hline $\begin{array}{l}\text { SRS } \\
\text { North }\end{array}$ & \begin{tabular}{|l} 
SRS \\
East
\end{tabular} & $\begin{array}{l}\text { Elevation } \\
\mathrm{ft} \mathrm{msl}\end{array}$ & \begin{tabular}{|l} 
SRS \\
North
\end{tabular} & $\begin{array}{l}\text { SRS } \\
\text { East }\end{array}$ & $\begin{array}{l}\text { Elevation } \\
\mathrm{ft} \mathrm{msl}\end{array}$ & $\begin{array}{l}\text { SRS } \\
\text { North }\end{array}$ & $\begin{array}{l}\text { SRS } \\
\text { East }\end{array}$ & $\begin{array}{l}\text { Elevation } \\
\mathrm{ft} \mathrm{msl}\end{array}$ \\
\hline \begin{tabular}{|c|c|}
102830.4 \\
\end{tabular} & 48863.39 & 295.3 & 102601.5 & 48738.86 & 262.97 & 102593.5 & 48671.42 & 321.854 \\
\hline 102839.4 & 48867.8 & 294.21 & 102599.1 & 48737.19 & 262.17 & 102602.8 & 48672.69 & 321.854 \\
\hline 102848.2 & 48872.38 & 293.12 & 102596.7 & 48735.5 & 261.45 & 102613.9 & 48673.99 & 321.854 \\
\hline 102856.9 & 48877.17 & 291.99 & 102590.4 & 48730.86 & 259.85 & 102622.2 & 48675.65 & 322.2943 \\
\hline 102865.6 & 48881.89 & 290.77 & 102586.5 & 48727.87 & 258.98 & 102632.5 & 48677.52 & 322.9664 \\
\hline 102874.1 & 48887.05 & 289.55 & 102582.6 & 48724.91 & 258.24 & 102642.6 & 48679.87 & 323.8586 \\
\hline 102882.3 & 48892.74 & 288.68 & 102578.6 & 48721.97 & 257.68 & 102652.8 & 48682.99 & 324.971 \\
\hline 102890.6 & 48898.24 & 287.98 & 102574.5 & 48719.04 & 257.37 & 102662.1 & 48686.35 & 326.3036 \\
\hline 102899 & 48903.57 & 287.06 & 102570.4 & 48716.18 & 257.2 & 102671.4 & 48689.91 & 327.6477 \\
\hline 102907.4 & 48908.89 & 286.02 & 102566.3 & 48713.32 & 257.07 & 102680.6 & 48693.71 & 329.8725 \\
\hline 102915.8 & 48914.21 & 284.89 & 102562.2 & 48710.46 & 257.02 & 102690.8 & 48697.62 & 332.3175 \\
\hline 102918.3 & 48915.8 & 284.55 & 102558.1 & 48707.68 & 257.04 & 102699.2 & 48701.78 & 335.4461 \\
\hline & & & 102553.9 & 48704.96 & 257.09 & 102707.5 & 48706.12 & 338.5632 \\
\hline & & & 102549.6 & 48702.33 & 257.15 & 102717.7 & 48709.89 & 342.1321 \\
\hline & & & 102545.3 & 48699.75 & 257.24 & 102725.1 & 48713.16 & 347.0336 \\
\hline & & & 102541 & 48697.31 & 257.33 & 102734.3 & 48716.03 & 351.7034 \\
\hline & & & 102536.6 & 48694.97 & 257.46 & 102742.7 & 48718.22 & 356.6165 \\
\hline & & & 102532.1 & 48692.72 & 257.68 & 102749.2 & 48720.36 & 360.8459 \\
\hline & & & 102527.6 & 48690.61 & 257.96 & 102756.6 & 48722.49 & 364.8552 \\
\hline & & & 102523 & 48688.56 & 258.27 & & & \\
\hline & & & 102518.5 & 48686.46 & 258.57 & & & \\
\hline & & & 102514 & 48684.36 & 258.88 & & & \\
\hline & & & 102509.5 & 48682.14 & 259.16 & & & \\
\hline & & & 102505.1 & 48679.8 & 259.4 & & & \\
\hline & & & 102500.7 & 48677.35 & 259.6 & & & \\
\hline & & & 102496.4 & 48674.79 & 259.84 & & & \\
\hline & & & 102492.2 & 48672.16 & 260.19 & & & \\
\hline & & & 102488 & 48669.54 & 260.53 & & & \\
\hline & & & 102483.7 & 48666.93 & 260.84 & & & \\
\hline & & & 102479.4 & 48664.34 & 261.1 & & & \\
\hline & & & 102475.2 & 48661.77 & 261.21 & & & \\
\hline & & & 102470.8 & 48659.32 & 261.12 & & & \\
\hline & & & 102466.4 & 48656.98 & 260.86 & & & \\
\hline & & & 102461.9 & 48654.81 & 260.45 & & & \\
\hline & & & 102457.4 & 48652.72 & 260.03 & & & \\
\hline & & & 102452.8 & 48650.78 & 259.75 & & & \\
\hline & & & 102448.1 & 48649.08 & 259.66 & & & \\
\hline & & & 102443.4 & 48647.42 & 259.82 & & & \\
\hline
\end{tabular}




\section{Appendix F - Barometric Pumping Mass Removal Rates}

Joe Rossabi

Mass removal for wells under going passive soil vapor extraction (barometric pumping) are governed by the same rules as active soil vapor extraction systems. Essentially, the mass removal rate is equal to the flow rate from the well multiplied by the concentration of the soil gas coming from the well. The flow rate from a well is governed primarily by the effective permeability of the formation in which the well is screened and the magnitude of the pressure differential causing the flow. Generally, the pressure difference caused by barometric effects is much lower than the pressure difference created by an active pumping system so flow is generally lower. However, barometric pumping can produce flow rates as high as $50 \mathrm{scfm}$ (measured at SRS in 2000). If the soil gas concentration is also high, a significant amount of contaminant can be removed by barometric pumping (see figure below). The flow rate from barometric pumping wells ranges from as low as $0.1 \mathrm{scfm}$ to as high as $50 \mathrm{scfm}$. At SRS, the barometric wells flow rate generally ranges between $0.5 \mathrm{scfm}$ and $10 \mathrm{scfm}$. At the Miscellaneous Chemical Basin and the MetLab Baroball well installations for example, the average flow rate from the 2 inch diameter wells is 2 scfm when they are blowing. The mass removal rate is calculated by multiplying the flow rate by the concentration of the soil gas in the well. The mass removal rate can vary over a very large range given the range of concentrations and flows that we have measured. The figure below shows the range of removal rates for tetrachloroethylene (PCE) based on measured concentrations and flows at SRS.

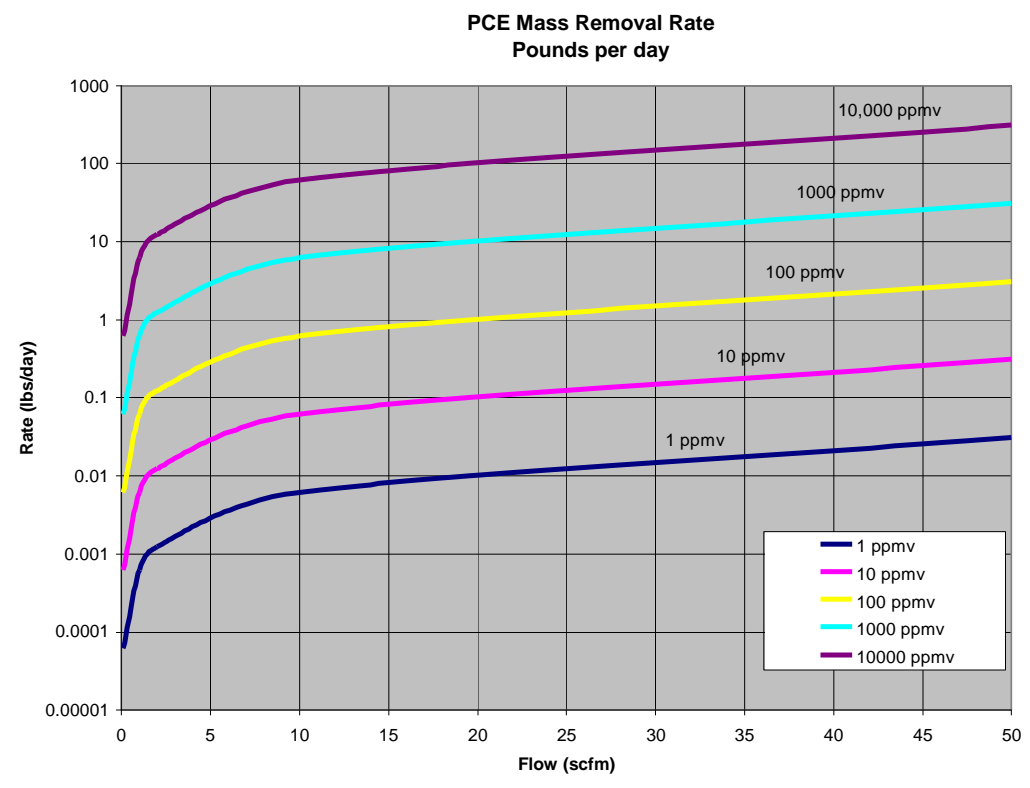

